\title{
First-Forbidden Beta Decay ${ }^{* \dagger}$
}

\author{
HANS A. WEIDENMÜLLER \\ California Institute of Technology, Pasadena, California
}

\section{INTRODUCTION}

$\mathbf{I}^{\mathrm{I}}$ $N$ the last few years study of the laws of $\beta$ decay has been the object of many experimental and theoretical investigations. As a consequence, the form of the nuclear $\beta$-decay interaction is now well-established. We know that $\beta$ decay violates parity conservation completely, and can be written $V-A$ for electron (negaton) emission (TEN 58). ${ }^{1}$

The fact that parity is not conserved has enlarged the number of possible experiments on nuclear $\beta$ decay: The measurement of the correlation between the direction of the $\beta$ particle and of the circular polarization of photons emitted after the $\beta$ decay, for example, has become a new source of information. This increase in number of experimental possibilities, together with our knowledge of the interaction law, has given nuclear $\beta$ decay a new aspect: It can be applied to the study of nuclear structure. In the same way in which we can use knowledge of the electrodynamic interaction to measure $E 1, M 1$ matrix elements, etc., we can use knowledge of the $\beta$ interaction in many cases to measure the nuclear matrix elements involved in the transition.

For the case of allowed transitions, where there are only two nuclear matrix elements $\int 1$ and $\int \sigma$, this scheme has been applied successfully in many cases. Recently, a combination of measurements of half-life, transition energy, spectrum shape factor, $\beta-\gamma$ angular correlation and $\beta$-circularly polarized $\gamma$-angular correlation has given a unique determination of the nuclear parameters involved in the first-forbidden $\beta$ decay of $\mathrm{Sb}^{124}$ (HA 60, ST 60). Similarly, a study of the spectrum of the first-forbidden $\beta$ decay of $\operatorname{Pr}^{144}$ and its $\log$ - $\mathrm{ft}$ value has yielded values for the two nuclear matrix elements relevant for the transition (PO 59).

For allowed transitions, Konopinski (KON 59) has recently given a summary of the present status of our knowledge; no such account exists for forbidden $\beta$ decay. In many recent papers, various aspects of forbidden $\beta$ decay have been discussed. Particularly, the papers of Kotani [e.g., (KOT 59b)] have dealt with the problems encountered in the investigation of nuclei by studying first-forbidden $\beta$ decay. However, access to these papers can often be obtained only by studying the classical papers on $\beta$ decay, as, for example, Konopinski (KON 41).

In the light of these developments and in view of the fact that in various laboratories efforts are being made to study nuclear matrix elements in first-forbidden

* The survey of the literature was closed on October 1, 1960. $\uparrow$ This work was performed under the auspices of the U. S. Atomic Energy Commission.

${ }^{1}$ For these references, see Bibliography at the end of the article. $\beta$ decay it is felt that a consistent presentation and discussion of the theoretical description of forbidden $\beta$ decay may be desirable.

The purpose of this paper is threefold: It attempts to give a concise introduction to the theoretical treatment of forbidden $\beta$ decay, it presents a review of the present status of the theory of first-forbidden $\beta$ decay, and it tries to give a full discussion and explanation of how nuclear $\beta$ decay matrix elements can be obtained.

The paper consists of two parts. The first part deals with the theoretical treatment of forbidden $\beta$ decay. It is assumed that the interaction is purely vector and axial vector, and that the two-component theory of the neutrino holds. Invariance under time reversal is an additional assumption used throughout the paper. The first three sections are devoted to a presentation of the methods and approximations used in the theoretical treatment of forbidden $\beta$ decay. They contain all the important steps in the derivation of the final formulas, but give rather qualitative accounts of the methods. They are supported by appendixes in which all the formulas and their derivations are given. The method followed in this part is the classical one [see, e.g., (ALD 57) ], although some of the appendixes contain material somewhat new in presentation. We hope that this splitting of the subject matter into main part and appendixes may simplify the access to the theory.

The second part is a review of recent theoretical literature, with the main emphasis on ways to study nuclear matrix elements.

Section 4 discusses the information available from various experimental investigations; particularly, derivations from the $\xi$ approximation are discussed because these cases furnish especially useful information about nuclear structure. Section 7 describes three examples which are typical of first-forbidden $\beta$ decay, and Sec. 8 demonstrates the variety of values the experimental parameters can assume. These chapters are supported by Appendix IV, where the more important formulas for experimental investigations are given, together with Tables III, IV, and VI, where the values for the characteristic parameters are listed. Using this information, one can immediately write formulas for spectrum shape, electron polarization, $\beta-\gamma$ correlation functions including various polarization measurements, and angular distribution of electrons and subsequently emitted $\gamma$ rays from oriented nuclei. The following experiments have not been considered: Neutrino correlation (recoil) experiments, correlation experiments where the polarization of both particles is measured, 
triple correlations, and correlations whereby intermediate $\gamma$ rays are not observed.

Preceding the Bibliography, a number of references (called "general references") to papers which contain formulas useful for the evaluation of experiments is given. Our formulas do not include third-order forbidden terms, finite nuclear size, or screening corrections.

The rest of the review sections gives a discussion of approximations better than the Konopinski-Uhlenbeck approximation (Appendix VI) of attempts to calculate the nuclear matrix elements theoretically (Sec. 5), and of the pseudoscalar interaction (Sec. 6). Although nowhere else in the text is reference to this interaction made, a discussion of the present evidence against its existence is a necessary part of a paper on first-forbidden $\beta$ decay. Except for Appendix VII, this paper does not deal with $K$ capture.

A recent review of the $K$ capture process has been published by Brysk and Rose (BR 58).

\section{NUCLEAR MATRIX ELEMENTS}

Since the discovery of parity nonconservation in $\beta$ decay, the laws of $\beta$ decay have been clarified by various experiments, and the form of the $\beta$ decay interaction is now well-established to be (KON 59 and references quoted therein)

$$
\begin{aligned}
H_{\beta}=\sum_{i} \sum_{\mu} \int\left[\psi_{f}{ }^{*} \gamma_{4}{ }^{(i)} \gamma_{\mu}{ }^{(i)}\left(C_{V}-C_{A} \gamma_{5}{ }^{(i)}\right) \tau_{i}{ }^{-} \psi_{i}\right] \\
{\left[\psi_{e}{ }^{*} \gamma_{4} \gamma_{\mu}\left(1+\gamma_{5}\right) \psi_{\nu}\right] d \tau+\text { herm. conj. } }
\end{aligned}
$$

where $H_{\beta}$ is the interaction density, $\psi_{i}$ and $\psi_{f}$ are the initial and final wave functions, $C_{V}$ and $C_{A}$ the vectorand axial-vector-coupling constants with the values (BA 60)

$$
\begin{aligned}
C_{V} & =(1.415 \pm 0.004) \times 10^{-49} \mathrm{erg} \mathrm{cm}^{3} \\
C_{A} / C_{V} & =-1.19 \pm 0.04 .
\end{aligned}
$$

The index $i$ refers to the nucleons building up the initial and final wave functions. $\psi_{e}$ and $\psi_{\nu}$ are the wave functions for electron and neutrino, respectively. The $\gamma$ matrices are defined in Appendix VIII. The above form of the interaction has the following meaning: The "lepton current"

$$
L_{\mu}(\mathbf{r})=\left[\psi_{e}^{*} \gamma_{4} \gamma_{\mu}\left(1+\gamma_{5}\right) \psi_{\nu}\right]
$$

is a four vector, dependent on a space coordinate $\mathbf{r}$. The bracket only means that the scalar product with respect to the spinor indices has to be taken. $L_{\mu}(\mathbf{r})$ also depends on the magnetic quantum numbers of electron and neutrino.

This "lepton current" interacts with the "baryon current"

$$
B_{\mu}(\mathbf{r})=\left[\psi_{f}^{*} \gamma_{4} \gamma_{\mu}\left(C_{V}-C_{A} \gamma_{5}\right) \tau^{-} \psi_{i}\right]
$$

and the form of the interaction is

$$
\sum_{\mu} \int B_{\mu}(\mathbf{r}) L_{\mu}(\mathbf{r}) d \tau
$$

If we compare this with the interaction of an electromagnetic current $j_{\mu}(\mathbf{r})$ with the electromagnetic field, given by its vector potential $A_{\mu}(\mathbf{r})$, we see that this interaction

$$
\sum_{\mu} \int j_{\mu}(\mathbf{r}) A_{\mu}(\mathbf{r}) d \tau
$$

has a'similarity to the $\beta$-decay interaction: In each case two four-vectors have a point interaction. It is, therefore, useful to consider first the approximations made in the simpler case of $\gamma$ radiation.

Here, the usual procedure consists in a multipole expansion of the vector potential of the radiation field, which is suggested by the fact that the nuclear levels can be characterized by their total spin $J$. It is justified because a multipole of order $L$ has a factor $(k r)^{L}$ in the expansion, where $k$ is the $\gamma$ energy and $r$ in the interaction integral is limited by the spatial extension of the current, that is, by the nuclear radius $R$. It turns out that $(k R) \ll 1$ for nuclear $\gamma$ transitions so that only multipoles of lowest possible order have to be considered.

Usually, keeping the lowest order terms amounts to keeping two types of matrix elements, e.g., the $M 1-$ and $E 2$ - matrix elements in the simplest case. One of them (in our case, the $M 1-$ matrix element) is of order $v / c$ in the nucleon velocities, compared to the leading electric dipole term, the other one (in our case the $E 2-$ matrix element) is of order $k R$ compared to the leading term, where $R$ is the nuclear radius. It so happens that $v / c$ and $k R$ are of the same order of magnitude for many transitions and therefore in many nuclei $M 1-$ and $E 2-$ transitions have comparable widths.

In nuclear $\beta$ decay, we find exactly the analogous situation, with a few modifications due to two facts: $L_{\mu}$ is not divergenceless and we have two types of interaction-vector and axial vector-rather than one, which increases the number of pertinent nuclear matrix elements.

$A_{\mu}$ is a divergenceless quantity with a gaugeinvariant interaction. This has the consequence that there are no electromagnetic monopole transitions (except by pair creation). (The photon always carries spin one.) The same statement does not hold for $L_{\mu}$, and the transitions corresponding to the electric monopole case are called allowed transitions in $\beta$ decay. The corresponding matrix elements are obtained by replacing the wavefunction of electron and neutrino by one-this corresponds to replacing $e^{i k r}$ by one in the electromagnetic case-and taking the nonrelativistic part of the interaction for the nucleus. Since in this case we are dealing with two interactions, we will be left with two nuclear matrix elements: $C_{V} \gamma_{4} \gamma_{\mu}$ is to be 
'TABLE I. Allowed and first-forbidden nuclear matrix elements and their selection rules ( $K$ designates the rank of the transition operator, when regarded as a tensor).

\begin{tabular}{|c|c|c|c|c|}
\hline \multicolumn{2}{|c|}{ Matrix element } & \multirow{2}{*}{$\frac{K}{0}$} & \multirow[t]{2}{*}{$\Delta J$} & \multirow{2}{*}{$\begin{array}{r}\Delta \pi \\
+1\end{array}$} \\
\hline Allowed & $C_{V} \int 1$ & & & \\
\hline & $C_{A} \int \boldsymbol{0}$ & 1 & $0, \pm 1($ no $0 \rightarrow 0)$ & +1 \\
\hline \multirow[t]{4}{*}{$\begin{array}{l}\text { First } \\
\text { for- } \\
\text { bidden }\end{array}$} & $\left.\begin{array}{l}C_{A} \int \gamma_{5} \\
C_{A} \int(\boldsymbol{\sigma} \cdot \mathbf{r} / i)\end{array}\right\}$ & 0 & 0 & -1 \\
\hline & $C_{V} \int \mathrm{r} i$ & & & \\
\hline & $\left.\begin{array}{l}C_{V} \int \boldsymbol{\alpha} \\
C_{A} \int(\boldsymbol{\alpha} \times \mathbf{r})\end{array}\right\}$ & 1 & $0, \pm 1($ no $0 \rightarrow 0)$ & -1 \\
\hline & $C_{A} \int i B_{i j}$ & 2 & $\begin{array}{c}0, \pm 1, \pm 2(\text { no } 0 \rightarrow 0 \\
\text { no } 1 \rightarrow 0, \text { no } 0 \rightarrow 1)\end{array}$ & -1 \\
\hline
\end{tabular}

replaced by $C_{V}$ (that is, we drop the first three components of the current) and $-C_{A} \gamma_{4} \gamma_{\mu} \gamma_{5}$ by $i C_{A} \boldsymbol{\sigma}$ (we drop the fourth component of the current). We have to deal with "Fermi transitions", characterized by the nuclear matrix element $C_{v} \int 1,{ }^{2}$ and the selection rule $\Delta J=0$, $\Delta \pi=+1$, and with "Gamow-Teller transitions," characterized by the nuclear matrix element $C_{A} \int \boldsymbol{\delta}$, and the selection rule $\Delta J=0, \pm 1($ no $0 \rightarrow 0) \Delta \pi=+1$.

The first nonvanishing term in the multipole expansion of $A_{\mu}$ is the dipole term. It has a matrix element which we can briefly denote by $\int \mathbf{r}$, and it is (neglecting the retardation) of order $k R$ and obeys the selection rule $\Delta J=0, \pm 1($ no $0 \rightarrow 0), \Delta \pi=-1$. The corresponding terms in the expansion of $L_{\mu}$ lead to the matrix elements for first-forbidden $\beta$ decay.

Inspection of Eq. (1) shows that there are many more matrix elements than just the analog of the $E 1$ - matrix element. Let us consider first the vector interaction. It consists of two parts, $\boldsymbol{\alpha}$ and 1, if we take the first three components of the current and the fourth one separately. The latter is an allowed term and contributed to the allowed transition. The former is of order $v / c$ in the nuclear coordinates, has the selection rule $\Delta J=0, \pm 1$, (no $0 \rightarrow 0), \Delta \pi=-1$ and is therefore a first-forbidden term. By keeping terms of order $q r$ and $k r$ in the lepton currents where $k$ and $q$ are electron and neutrino momentum respectively, the matrix element with the operator 1 becomes $\int \mathbf{r}$, which obeys the selection rules for first-forbidden decay. Keeping correspondingly terms of order $\mathbf{r}$ in the matrix element $\int \boldsymbol{\alpha}$ would lead to second-order-forbidden matrix elements, which we do not consider here. We see that there are two first-forbidden nuclear matrix elements originating from the vector interaction, $\int \boldsymbol{\alpha}$ and $\int \mathbf{r}$.

\footnotetext{
${ }^{2}$ It is customary to write the matrix elements for nuclear $\beta$ decay as integrals over the transition operators, omitting the symbols for the initial- and final-state wavefunctions. A complete definition of these matrix elements is given in the appendixes.
}

Correspondingly, the axial vector interaction consists of the two parts, $\boldsymbol{\sigma}$ and $\gamma_{5}$. The first term gives rise to an allowed matrix element if one replaces the lepton current by one. If one again keeps terms of order $q r$ and $k r$ in the lepton current, this interaction gives rise to the following three first-forbidden matrix elements: $\int \boldsymbol{\sigma} \cdot \mathbf{r}, \int[\boldsymbol{\delta} \times \mathbf{r}], \int B i j=\int\left[\sigma_{i} x_{j}+x_{i} \sigma_{j}-\frac{2}{3} \delta_{i j}(\boldsymbol{\sigma} \cdot \mathbf{r})\right]$. Here, $B_{i j}$ is a symmetric tensor of second rank with trace zero. This is a convenient form because the trace is already contained in $\int \boldsymbol{\delta} \cdot \mathbf{r}$. The matrix element $\int \gamma_{5}$ is already of first-forbidden type, being of order $v / c$.

The selection rules obeyed by the axial vector firstforbidden nuclear matrix elements are obvious: They all have $\Delta \pi=-1$, and $\int \gamma_{5}$ and $\int \boldsymbol{\sigma} \cdot \mathbf{r}$ have $\Delta J=0$, the operators being pseudoscalars, $\int[\sigma \times r]$ has $\Delta J=0, \pm 1$ (no $0 \rightarrow 0$ ), and $B_{i j}$ has $\Delta J=0$, $\pm 1, \pm 2$, (no $0 \rightarrow 0$, no $0 \rightarrow 1$, no $1 \rightarrow 0$ ).

The first-forbidden $\beta$ decay is, therefore, governed by six matrix elements. They are summarized in Table I, together with their selection rules.

Whereas in the case of an allowed decay, a simple interpretation of the physical process is possible by saying that neither electron nor neutrino carry away any orbital angular momentum and that their spins are in a singlet or triplet state for Fermi and GamowTeller interaction, respectively, this is no longer easily possible for forbidden transitions. One can, however, make the following remarks:

The lepton interaction connected with the matrix elements $\int \boldsymbol{\alpha}$ and $\int \gamma_{5}$ differs from the corresponding interaction for the allowed matrix elements $\int \boldsymbol{\delta}$ and $\int 1$ only in that it contains a multiplying factor $\gamma_{5}$. [We have, in both cases, taken the terms of order $(k r)^{\circ}$ in the expansion of the lepton wave functions.] Since, however, $L_{\mu}$ contains also a factor $\left(1+\gamma_{5}\right)$, and since $\gamma_{5} \cdot\left(1+\gamma_{5}\right) \equiv\left(1+\gamma_{5}\right), \quad L_{\mu}$ is invariant against this substitution. That is to say, the physical expressions involving $\int \boldsymbol{\alpha}$ and $\int \gamma_{5}$ can be obtained by taking the corresponding expressions for allowed $\beta$ decay, and making the replacements $C_{v} \int 1 \rightarrow-C_{A} \int \gamma_{5}, C_{A} \int \boldsymbol{\sigma} \rightarrow-C_{v} \int \boldsymbol{\alpha}$. Particularly, the simple interpretation described above for the allowed case applies also here.

In the case of the other four matrix elements, such a simple interpretation is not possible. In all of these cases, one may say that either electron or neutrino carries away one unit of orbital angular momentum, the other particle travelling away in an $s$ state. This statement is fallacious, however, because both electron and neutrino are relativistic particles, their "small" component having opposite parity to the "large" component for each eigenstate of $\mathbf{j}$, the total angular momentum. Therefore, a description in terms of eigenstates of $\mathbf{j}$ is more appropriate, and this is the way the calculation is actually done. However, one then leaves the ground of simple pictures of what is happening.

It is the objective of this report to derive expressions for various measurable quantities as bilinear functions of the six matrix elements given in Table I. This task 
is more complicated than in the case of electrodynamics because of the following reasons:

In an expression involving the matrix elements $\int \gamma_{5}$ and $\int \boldsymbol{\alpha}$, which are of order $v / c$, the lepton current may be treated in the "allowed" approximation, i.e., electron and neutrino wave functions may be replaced by their values for $r \rightarrow 0$. This does not hold for expressions involving the other four matrix elements, where the next term in the expansion of either the electron or the neutrino wave functions has to be taken. Therefore, the energy dependence of the resulting expressions is not homogeneous. (This energy dependence comes mainly from the electron and neutrino wave functions and their power-series expansions.)

The fact that electron and neutrino, two relativistic particles, constitute the lepton current which we want to expand in multipoles is another source of difficulties. We are not dealing with the wave function of one particle as in the electrodynamic case. Instead, the current is a rather complex algebraic expression in the two wave functions.

The third major difficulty arises from the fact that the electron is a charged particle and moves in the Coulomb field of the nucleus. This has a great bearing on the first-forbidden $\beta$ decay, as we see below, since the Coulomb interaction at the nuclear radius is of the order of a few Mev, and therefore of the same order or larger than the maximum energy of the $\beta$ 's. The distortion of the electron (or positron) wave function is appreciable, and in many cases more important than the next term of order $k r$ or $q r$ in the power-series expansion of these functions.

Summarizing we see that the various "forbidden" matrix elements in $\beta$ decay occur by expanding the lepton current $L_{\mu}$ into its multipoles, and by taking into account of the nuclear matrix elements of order $v / c$ in the appropriate manner. Generally speaking, the $n$ thforbidden $\beta$ decay $(n>1)$ is characterized by matrix elements of two types:

$$
\int Y_{n} r^{n} x\left\{\begin{array}{l}
1 \\
\boldsymbol{\delta}
\end{array}\right\} d \tau, \int Y_{n-1} r^{n-1}\left\{\begin{array}{c}
\gamma_{5} \\
\boldsymbol{\alpha}
\end{array}\right\} d \tau,
$$

where $Y_{n}$ is the $n$th spherical harmonic, and by the selection rules $\Delta J= \pm n, \pm(n+1) ; \Delta \pi=(-)^{n}$.

\section{PARTICLE PARAMETERS}

The actual derivation of the density matrix and the various measurable quantities as functions of the six matrix elements and the energy and momentum of electron and neutrino are given in Appendixes III and IV; in this chapter we give a brief outline of the procedure and the results in order to allow the reader to become familiar with the subject without going through the algebra.

The lepton current $L_{\mu}$ involves the relativistic wave functions for electron and neutrino; it must be the first task to write these functions properly. These continuum functions are the analogs to a plane wave in the case of a nonrelativistic particle of spin zero. Similar to the Rayleigh expansion of a plane wave, which is an expansion in eigenfunctions of the orbital angular momentum of the particle, they are given as a sum of eigenfunctions of the operator $\kappa_{\mathrm{op}}$, which specifies the total spin $j$ and the parity $\pi$ of the state. In Appendix I, we repeat briefly the derivation of these eigenfunctions, which are solutions to the field-free case for the neutrino and to the Coulomb-field case for the electron. The complete wave functions are given in Appendix III in terms of the definitions used in Appendix I. The eigenvalues of the operator $\kappa_{\mathrm{op}}$ are all integers $\kappa$ except zero, and are related to the total spin by

$$
\kappa^{2}=\left(j+\frac{1}{2}\right)^{2}
$$

and to the angular momentum of the "larger component" by

$$
\begin{aligned}
& \kappa>0: \kappa=l \\
& \kappa<0: \kappa=-l-1 .
\end{aligned}
$$

To each eigenfunction to $\kappa_{\mathrm{op}}$, there belong two radial functions $f_{\kappa}$ and $g_{\kappa}$, the "large" and "small" radial wave functions, which are simple Bessel functions in the case of the neutrino, and more complicated expressions for the case of the Coulomb field. They are given in Appendix I.

As a next step we confine attention to a particular coordinate system and decompose the expressions for $L_{\mu}$ and $B_{\mu}$ losing, of course, the apparent relativistic invariance of the theory. In this coordinate system (which we still can specify), the interaction density has the form (here and in the following we suppress the summation over the nucleons $i$ with the understanding that in all our expressions this sum has to be carried along) :

$$
\begin{array}{r}
M_{\beta}=\int\left[\psi_{f}^{*}\left(C_{V}-C_{A} \gamma_{5}\right) \tau^{-} \psi_{i}\right]\left[\psi_{e}^{*}\left(1+\gamma_{5}\right) \psi_{\nu}\right] d \tau \\
-\int\left[\psi_{f}^{*}\left(C_{V}-C_{A} \gamma_{5}\right) \boldsymbol{\alpha} \tau^{-} \psi_{i}\right]\left[\psi_{e}^{*} \boldsymbol{\alpha}\left(1+\gamma_{5}\right) \psi_{\nu}\right] d \tau \\
+ \text { herm. conj. }
\end{array}
$$

We rewrite this in the form,

$$
\begin{aligned}
M_{\beta}=\int d \tau_{1} \int d \tau_{2}\left[\psi_{f}^{*}\left(C_{V}-C_{A} \gamma_{5}\right) \tau^{-} \psi_{i}\right]_{\mathbf{r}=\mathbf{r}_{1}} \\
\cdot\left[\psi_{e}{ }^{*}\left(1+\gamma_{5}\right) \psi_{v}\right]_{\mathbf{r}=\mathbf{r}_{1}} \delta\left(\mathbf{r}_{1}-\mathbf{r}_{2}\right)+\cdots,
\end{aligned}
$$

and use the expansion

$$
\begin{aligned}
\delta\left(\mathbf{r}_{1}-\mathbf{r}_{2}\right)=\left(r_{1} r_{2}\right)^{-1} \delta\left(r_{1}-r_{2}\right) \sum_{L, M}(-)^{L+M} \\
\cdot\left[i^{L} Y_{L}^{M}\left(\mathbf{r}_{0}^{1}\right)\left(i^{L} Y_{L}^{-M}\left(\mathbf{r}_{0}^{2}\right)\right],\right.
\end{aligned}
$$

where $\mathbf{r}_{0}{ }^{i}=\mathbf{r}_{i} / \boldsymbol{r}_{i}$. The use of this expansion has the advantage that we can carry through the integration over the angles in the lepton current separately. Clearly, for first-forbidden transitions only the terms with $L=0,1$ contribute, as one sees by looking at Table 1 . The remaining radial integration involves the nuclear wave functions and the radial wave functions of electron and neutrino. 
TABLE II. Expressions for the $\beta$-decay multipole operators in cartesian coordinates, for vector-and axial-vector interaction and al lowed and first-forbidden transitions. The first column gives the reduced matrix element of the multipole operator, the second column its equivalent in cartesian coordinates, based on the definition (E. 3).

\begin{tabular}{|c|c|c|}
\hline \multirow[t]{2}{*}{ Allowed case } & {$\left[2 J_{i}+1\right]^{-\frac{1}{2}}\left\langle J_{f}\left\|C_{V} T_{000} \tau^{-}\right\| J_{i}\right\rangle$} & {$[4 \pi]^{-\frac{1}{2}} C_{V} \int 1$} \\
\hline & {$\left[2 J_{i}+1\right]^{-\frac{1}{2}}\left\langle J_{f}\left\|C_{A \gamma_{5}} T_{101} \tau^{-}\right\| J_{i}\right\rangle$} & {$[4 \pi]^{-\frac{1}{2}} C_{A} \int \boldsymbol{\sigma}$} \\
\hline \multirow[t]{6}{*}{ First-forbidden case } & {$\left[2 J_{i}+1\right]^{-\frac{1}{2}}\left\langle J_{f}\left\|C_{A \gamma_{5}} T_{000} \tau^{-}\right\| J_{i}\right\rangle$} & {$[4 \pi]^{-\frac{1}{2}} C_{A} \int \gamma_{5}$} \\
\hline & {$\left[2 J_{i}+1\right]^{-\frac{1}{2}}\left\langle J_{f}\left\|C_{V} T_{110 \tau^{-}}\right\| J_{i}\right\rangle$} & {$[3 / 4 \pi]^{\frac{1}{2}} C_{V} \int i(\mathbf{r} / r)$} \\
\hline & {$\left[2 J_{i}+1\right]^{-\frac{1}{2}}\left\langle J_{f}\left\|C_{V} T_{101} \tau^{-}\right\| J_{i}\right\rangle$} & {$[4 \pi]^{-\frac{1}{2}} C_{V} \int \boldsymbol{\alpha}^{\mathrm{a}}$} \\
\hline & {$\left[2 J_{i}+1\right]^{-\frac{1}{2}}\left\langle J_{f}\left\|C_{A \gamma_{5}} T_{011} \tau^{-}\right\| J_{i}\right\rangle$} & {$[4 \pi]^{-\frac{1}{2}} C_{A} \int[\boldsymbol{\sigma}(\mathbf{r} / r)] i^{-1}$} \\
\hline & {$\left[2 J_{i}+1\right]^{-\frac{1}{2}}\left\langle J_{f}\left\|C_{A} \gamma_{5} T_{111} \tau^{-}\right\| J_{i}\right\rangle$} & {$[3 / 8 \pi]^{\frac{1}{2}} C_{A} \int[\boldsymbol{\sigma} \times(\mathbf{r} / r)]^{\mathrm{b}}$} \\
\hline & {$\left[2 J_{i}+1\right]^{-\frac{1}{2}}\left\langle J_{f}\left\|C_{A \gamma_{5}} T_{211} \tau^{-}\right\| J_{i}\right\rangle$} & {$[3 / 2]^{\frac{1}{2}}[8 \pi]^{-\frac{1}{2}} C_{A} \int i B_{i j} / r^{\mathrm{c}}$} \\
\hline
\end{tabular}

a This differs from the corresponding entry in the table of Alder, Stech, and Winther (ALD 57) because our $\boldsymbol{\alpha}$ is by definition equal to minus the $\alpha$ of these authors.

b This differs from the corresponding entry in the table of Alder, Stech, and Winther (ALD 57) because of the reversed order of angular momentum coupling in their $\phi_{\Lambda L}$ and our $T_{K L} \boldsymbol{\gamma}$.

c Here, $\int_{i B_{i j / r}}=\int^{K}(i / r)\left(\sigma_{i} x_{j}+\sigma_{j} x_{i}-\frac{2}{3} \delta_{i j}(\boldsymbol{d r})\right.$ [see e.g., (KON 41)].

In the lepton as well as in the baryon currents the spherical harmonics coming from the expansion of the delta-function in (2.5) combine with the operators 1 or $\boldsymbol{\alpha}$ in (2.3) and (2.4) to "irreducible tensors"

and

$$
T_{L L 0}{ }^{M}=i^{L} Y_{L}^{M}
$$

$$
T_{K L 1}{ }^{M}=\sum_{v} c(1 L K ; M-v, v) \sigma^{M-v}\left(i^{L} Y_{L^{v}}\right) \gamma_{5}
$$

Having rewritten the interaction in terms of these irreducible tensors, it is easy to apply the Wigner-Eckart theorem and express all measurable quantities in terms of the reduced matrix elements of these irreducible tensors and geometrical coefficients. (Appendixes III and IV.)

The next step consists in setting up the "density matrix" of the interaction. We take the matrix element of $H_{\beta}$ between lepton and baryon states of specified magnetic quantum number and denote it by

$$
\left\langle J_{f}, m_{p} m_{e}\left|H_{\beta}\right| J_{i}, m_{i} m_{\nu}\right\rangle \text {. }
$$

The density matrix is defined by

$$
\begin{aligned}
\rho\left(m_{p}, m_{p}{ }^{\prime} ; m_{n}, m_{n}{ }^{\prime} ; m_{e}, m_{e}{ }^{\prime}, m_{\nu}, m_{\nu}{ }^{\prime}\right) & \\
= & \left\langle J_{f}, m_{p} m_{e}\left|H_{\beta}\right| J_{i}, m_{n} m_{\nu}\right\rangle \\
& \left\langle J_{f}, m_{p}{ }^{\prime} m_{e}{ }^{\prime}\left|H_{\beta}\right| J_{i}, m_{n}{ }^{\prime} m_{\nu}{ }^{\prime}\right\rangle^{*}
\end{aligned}
$$

where $m_{p}, m_{p}{ }^{\prime}$ are the magnetic quantum numbers of the final, $m_{n}$ and $m_{n}^{\prime}$ the magnetic quantum numbers of the initial state, and $m_{e}, m_{e}{ }^{\prime}$ and $m_{\nu}, m_{\nu}{ }^{\prime}$ refer to electron and neutrino, respectively. Since we are not going to consider recoil experiments we sum over $m_{\nu}=m_{\nu}{ }^{\prime}$ and integrate over all directions of the neutrino.

From the resulting expression, we can calculate formulas for the spectrum, the electron polarization from unoriented nuclei, the $\beta-\gamma$ angular correlation functions, etc. The procedure involves Racah techniques.
The expressions derived this way can be written as a product of one or two $F$ coefficients with one $B$ coefficient, a typical case being the angular distribution of electrons from oriented nuclei, which is given by

$$
\begin{aligned}
& A\left(\theta_{e}\right)=16 \pi \sum_{K K^{\prime} J} h_{J}\left(J_{i}\right)(-)^{K+K^{\prime}+J} F_{J} J^{0, J} \\
& \cdot\left(K, K^{\prime} ; J_{f}, J_{i}\right) B_{J}^{J, 0}\left(K, K^{\prime} ; J_{f}, J_{i}\right) P_{J^{0}}\left(\theta_{e}\right),
\end{aligned}
$$

where $h_{J}\left(J_{i}\right)$ specifies the initial polarization, and $\theta_{e}$ is the angle between the direction of polarization and the direction of the electron. In these expressions, $F_{J}{ }^{u, t}\left(K, K^{\prime} ; J_{f}, J_{i}\right)$ is a purely geometrical quantity; its index $J$ is usually connected with the angular distribution observed in the sense that with $F_{J}$ there comes a factor $P_{J}$, where $P_{J}$ is the Legendre polynominal of degree $J . J_{f}$ and $J_{i}$ denote the final and initial nuclear spin in the $\beta$ decay, $K$ and $K^{\prime}$ are numbers which we discuss below in connection with the $B$ 's, and $u$ and $t$ specify whether one observes-directly or indirectly-polarization or alignment of the initial and final nucleus: For polarized initial nuclei, $t$ takes all values between 0 and $J$, for unpolarized initial nuclei it is zero; and if one observes polarization or alignment of the final nucleus by looking at the angular distribution or circular polarization of subsequently emitted $\gamma$ radiation, $u$ runs from 0 to $J$, otherwise it is zero.

Whereas the quantities $F$ are purely geometrical in character, the quantities $B$ contain the dynamical information about the decay. $B_{J} u^{\prime t^{\prime}}$ depends on $J, u^{\prime}$, and $t^{\prime} . J$ has the same significance as in $F$. $t^{\prime}$ is zero if one does not measure the electron polarization, and one if it is measured. $u^{\prime}$ is an independent number if one does measure the electron polarization in coincidence with a subsequent radiation, otherwise it is zero or $J$. The quantities $B$ are often referred to as the particle parameters, because they are bilinear forms in both the nuclear and lepton reduced matrix elements 
FIRST-FORBID DEN B ETA DECAY

579

TABLE III. Values for the particle parameters $b_{J^{u, t}}^{u}\left(K, K^{1} ; J_{i}, J_{f}\right)$ for some cases of interest.

We introduce the following definitions:

$$
\begin{gathered}
L_{\kappa k^{\prime}}=f_{\kappa} f_{\kappa^{\prime}} \exp \left[i\left(\Delta_{\kappa}-\Delta_{\kappa^{\prime}}\right)\right]+g_{-\kappa} g_{-k^{\prime}} \exp \left[i\left(\Delta_{-\kappa}-\Delta_{-\kappa^{\prime}}\right)\right] \\
M_{\kappa k^{\prime}}=f_{\kappa} g_{-k^{\prime}} \exp \left[i\left(\Delta_{\kappa}-\Delta_{-\kappa^{\prime}}\right)\right]+g_{-\kappa} f_{\kappa^{\prime}} \exp \left[i\left(\Delta_{-\kappa}-\Delta_{+\kappa^{\prime}}\right)\right]
\end{gathered}
$$

and obtain for the parameters $b_{J}^{J, 0}\left(K, K^{1} ; J_{f}, J_{i}\right):{ }^{\mathrm{a}}$

$$
\begin{aligned}
& \begin{array}{llll}
J & K & K^{1}
\end{array} \\
& b_{J}^{J, 0}\left(K, K^{1} ; J_{f}, J_{i}\right) \\
& 0 \quad 0 \quad 0 \quad \frac{1}{2} C_{A^{2}}\left(\int \frac{(\boldsymbol{\sigma} \cdot \mathbf{r})}{i r}\right)^{2} L_{11}+\left[\frac{2}{-2} q R C_{A^{2}}\left(\int \frac{(\boldsymbol{\delta} \cdot \mathbf{r})}{i r}\right)^{2}+2 C_{A^{2}} \int \gamma_{5} \int \frac{\mathbf{\boldsymbol { \theta }} \cdot \mathbf{r}}{i r}\right] \operatorname{Re}\left(M_{1-1}\right) \\
& +\left[\frac{1}{18}(q R)^{2} C_{A^{2}}\left(\int \frac{(\boldsymbol{\delta} \cdot \mathbf{r})}{i r}\right)^{2}+\frac{1}{3}(q R) C_{A^{2}} \int \gamma_{5} \int \frac{\boldsymbol{0} \cdot \mathbf{r}}{i r}+\frac{1}{2} C_{A^{2}}\left(\int \gamma_{5}\right)^{2}\right] \operatorname{Re}\left(L_{1-1}\right)
\end{aligned}
$$

$0 \quad 1 \quad 1 \quad\left[\begin{array}{l}3 \\ -4\end{array} C_{A}{ }^{2}\left(\int \frac{(\mathbf{\sigma} \times \mathbf{r})}{r}\right)^{2}+\frac{3}{\sqrt{2}} C_{A} C_{V} \int \frac{i \mathbf{r}}{r} \int \frac{(\mathbf{\sigma} \times \mathbf{r})}{r}+3 C_{V} \int^{2} \frac{i \mathbf{r}}{r}\right] L_{11}$

$$
\begin{aligned}
& -\left[q R\left(\frac{1}{2} C_{A^{2}}\left(\int \frac{\boldsymbol{\sigma} \times \mathbf{r}}{r}\right)^{2}-C_{V}{ }^{2}\left(\int \frac{i \mathbf{r}}{r}\right)^{2}\right)-\sqrt{\left(\frac{3}{2}\right)} C_{A} C_{V} \int \alpha \int \frac{\boldsymbol{d} \times \mathbf{r}}{r}-\sqrt{3} C_{V^{2}} \int \alpha \int \frac{i \mathbf{r}}{r}\right] \operatorname{Re}\left(M_{1-1}\right) \\
& +\left[\frac{3}{4} C_{A^{2}}\left(\int \frac{\boldsymbol{\sigma} \times \mathbf{r}}{r}\right)^{2}-\frac{3}{\sqrt{2}} C_{A} C_{V} \int \frac{i \mathbf{r}}{r} \int \frac{\boldsymbol{\sigma} \times \mathbf{r}}{r}+6 C_{V^{2}}\left(\int \frac{i \mathbf{r}}{r}\right)^{2}\right] L_{-2-2} \\
& \left.+\left[(q R)^{2}\left(\frac{1}{4} C_{A^{2}}\left(\int \frac{\boldsymbol{0} \times \mathbf{r}}{r}\right)^{2}+C_{V^{2}}\left(\int \frac{i \mathbf{r}}{r}\right)^{2}\right)-\frac{2}{3} q R\left(C_{A} C_{V} \sqrt{\left(\frac{3}{2}\right.}\right) \int \boldsymbol{\alpha} \int \frac{\boldsymbol{\sigma} \times \mathbf{r}}{r}-\sqrt{3} C_{V^{2}} \int \boldsymbol{\alpha} \int \frac{i \mathbf{r}}{r}\right)\right] L_{-1-1}
\end{aligned}
$$

$0 \quad 2 \quad 2 \quad \frac{9}{32} C_{A^{2}}\left(\int \frac{B_{i j}}{r}\right)^{2}\left[\begin{array}{c}1 \\ L_{-2-2}+\frac{-}{9}(q R)^{2} L_{-1-1}\end{array}\right]$

$$
\begin{aligned}
& 1 \quad 0 \quad 1+\left[\frac{1}{\sqrt{ } 6} C_{A^{2}} \int \frac{\boldsymbol{\sigma} \times \mathbf{r}}{r} \int_{i r}^{\mathbf{\sigma} \cdot \mathbf{r}}+\frac{1}{\sqrt{3}} C_{A} C_{V} \int \frac{\boldsymbol{0} \cdot \mathbf{r}}{i r} \int \frac{i \mathbf{r}}{r}\right] \operatorname{Re}\left(M_{11}\right)
\end{aligned}
$$

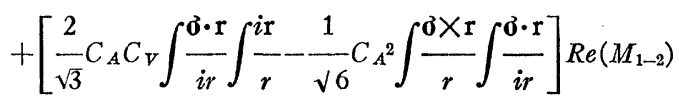

$$
\begin{aligned}
& +\left[\frac{2}{3 \sqrt{3}} q R C_{A} C_{V} \int \frac{(\boldsymbol{\sigma} \cdot \mathbf{r})}{i r} \int_{r}^{i \mathbf{r}}+\frac{1}{\sqrt{3}} C_{A} C_{V} \int \gamma_{5} \int_{r}^{i \mathbf{r}}+\frac{1}{\sqrt{ } 6} C_{A^{2}} \int \gamma_{5} \int \frac{\boldsymbol{\sigma} \times \mathbf{r}}{r}+\frac{1}{3} C_{A} C_{V} \int \frac{\boldsymbol{\delta} \cdot \mathbf{r}}{i r} \int \boldsymbol{\alpha}\right] R e\left(L_{1-1}\right) \\
& +\left[q R\left(\frac{2}{3 \sqrt{3}} C_{A} C_{V} \int \frac{(\boldsymbol{\sigma} \cdot \mathbf{r})}{i r} \int_{r}^{i \mathbf{r}}-\frac{1}{3 \sqrt{ } 6} C_{A^{2}} \int \frac{\boldsymbol{\sigma} \times \mathbf{r}}{r} \int \frac{(\boldsymbol{\sigma} \cdot \mathbf{r})}{i r}\right)+\frac{2}{3 \sqrt{3}} C_{A} C_{V} \int \gamma_{5} \int \frac{i \mathbf{r}}{r}-\frac{1}{3 \sqrt{ } 6} \int \gamma_{5} \int \frac{\boldsymbol{\sigma} \times \mathbf{r}}{r}\right] \\
& +\left[(q R)^{2}\left(\frac{1}{9 \sqrt{3}} C_{A} C_{V} \int_{i r}^{\boldsymbol{\delta} \cdot \mathbf{r}} \int_{r}^{i \mathrm{r}} \frac{1}{9 \sqrt{ } 6} C_{A^{2}} \int^{\boldsymbol{\delta} \times \mathbf{r}} \frac{(\boldsymbol{\sigma} \cdot \mathbf{r})}{i r}\right)\right. \\
& \operatorname{Re}\left(L_{-1-2}\right) \\
& \left.+(q R)\left(\frac{1}{3 \sqrt{3}} C_{A} C_{V} \int \gamma_{5} \int \frac{i \mathbf{r}}{r}-C_{A^{2}} \frac{1}{3 \sqrt{ } 6} \int \gamma_{5} \int \frac{\boldsymbol{\sigma} \times \mathbf{r}}{r}+\frac{1}{9} C_{A} C_{V} \int \frac{\boldsymbol{\sigma} \cdot \mathbf{r}}{i r} \int \boldsymbol{\alpha}\right)+\frac{1}{3} \int \gamma_{5} \int \boldsymbol{\alpha}\right] \operatorname{Re}\left(M_{-1-1}\right)
\end{aligned}
$$


580

HA NS A. WEIDENM ÜLLER

Table III (continued)

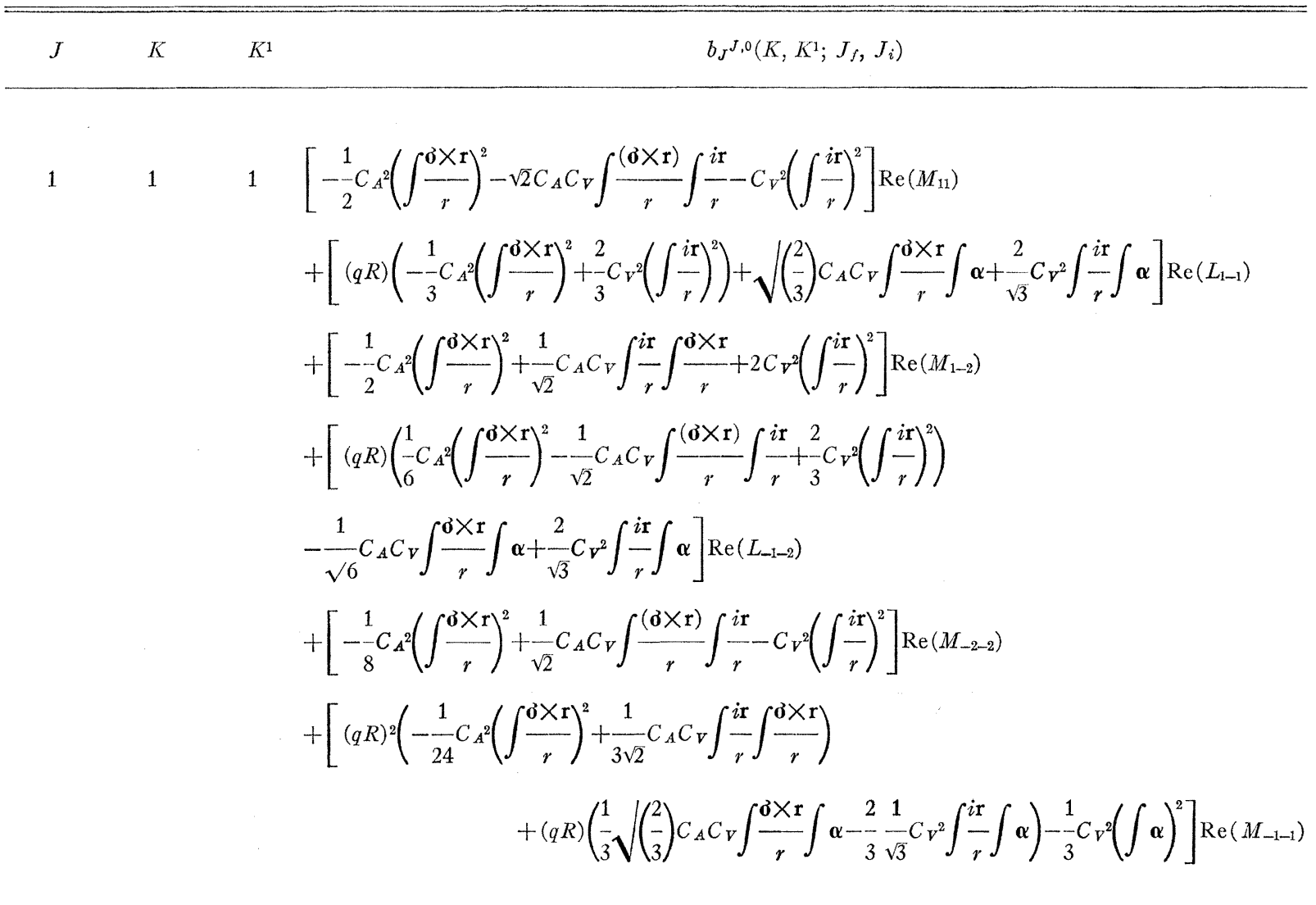

$1 \quad 1 \quad 2 \quad C_{A^{2}} \int \frac{B_{i j}}{r}\left\{\left[-\frac{\sqrt{15}}{4} C_{A} \int \frac{\boldsymbol{\sigma} \times \mathbf{r}}{r}+\frac{1}{2} \sqrt{\left(\frac{15}{2}\right) C_{V} \int_{r}^{i \mathbf{r}}}\right] \operatorname{Re}\left(M_{1-2}\right)\right.$

$$
\begin{aligned}
& \left.\left.+\left[(q R)\left(\frac{1}{4} \sqrt{\left(\frac{5}{3}\right.}\right) C_{A} \int \frac{\boldsymbol{\sigma} \times \mathbf{r}}{r}-\frac{1}{2} \sqrt{ }\left(\frac{5}{6}\right) C_{V} \int \frac{i \mathrm{r}}{r}\right)-\frac{1}{2} \sqrt{\left(\frac{5}{2}\right.}\right) C_{V} \int \boldsymbol{\alpha}\right] \operatorname{Re}\left(L_{-1-2}\right) \\
& +\left[\frac{1}{8} \sqrt{\left(\frac{3}{5}\right)} C_{A} \int \frac{\boldsymbol{\sigma} \times \mathrm{r}}{r}-\frac{1}{2} \sqrt{ }\left(\frac{3}{10}\right) C_{V} \int \frac{i \mathrm{r}}{r}\right] \operatorname{Re}\left(M_{-2-2}\right) \\
& \left.\left.+\left[(q R)^{2}\left(-\frac{1}{24} \sqrt{\left(\frac{5}{3}\right.}\right) C_{A} \int \frac{\mathbf{0} \times \mathbf{r}}{r}+\frac{1}{6} \sqrt{ }\left(\frac{5}{6}\right) C_{V} \int \frac{i \mathbf{r}}{r}\right)\right] \operatorname{Re}\left(M_{-1-1}\right)\right\}
\end{aligned}
$$

$1 \quad 2 \quad 2 \quad\left(\int \frac{B_{i j}}{r}\right)^{2}\left[-\frac{27}{160} \operatorname{Re}\left(M_{-2-2}\right)-\frac{3}{160}(q R)^{2} \operatorname{Re}\left(M_{-1-1}\right)\right]$

$2 \quad 0 \quad 2 \quad C_{A} \int i \frac{B_{i j}}{r}\left\{-\frac{3}{10 \sqrt{2}} C_{A} \int \frac{\mathbf{0} \cdot \mathbf{r}}{r} \operatorname{Re}\left(L_{-1-2}\right)+\left[-\frac{1}{10 \sqrt{2}}(q R) C_{A} \int \frac{\mathbf{0} \cdot \mathbf{r}}{i r}+\frac{3}{10 \sqrt{2}} C_{A} \int \gamma_{5}\right] \operatorname{Re}\left(M_{-1-2}\right)\right\}$ 


\begin{tabular}{|c|c|c|c|}
\hline$J$ & $K$ & $K^{1}$ & $b_{J}^{J, 0}\left(K, K^{1} ; J_{f}, J_{i}\right)$ \\
\hline 2 & 1 & 1 & 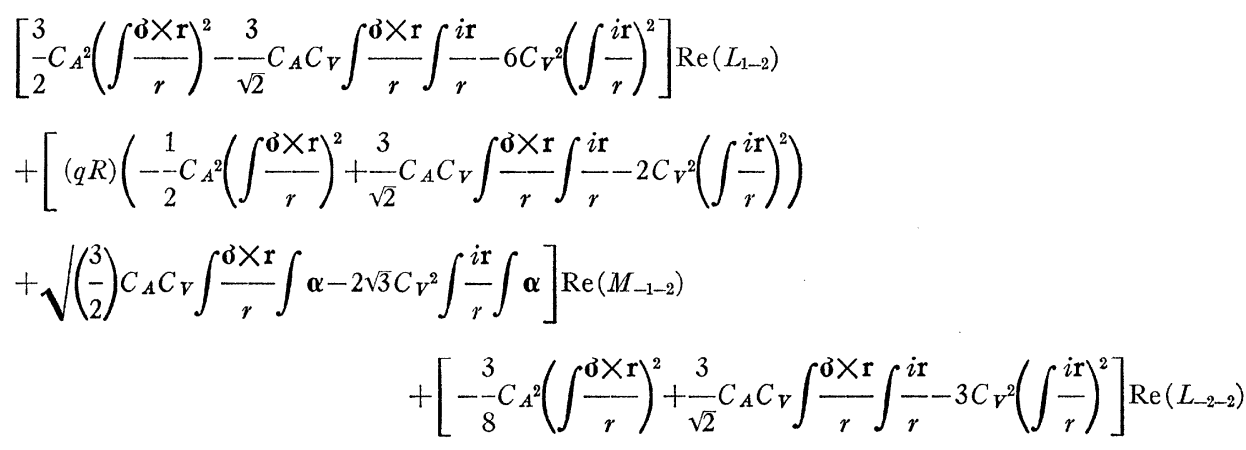 \\
\hline 2 & 1 & 2 & $\begin{array}{l}C_{A} \int \frac{B_{i j}}{r}\left\{\left[\frac{3}{2 \sqrt{ } 5} C_{A} \int \frac{\boldsymbol{\sigma} \times \mathbf{r}}{r}+\frac{3}{\sqrt{ } 10} C_{V} \int^{i \mathbf{r}} \frac{\operatorname{ra}}{r}\right] \operatorname{Re}\left(L_{1-2}\right)\right. \\
+\left[(q R)\left(\frac{1}{2 \sqrt{ } 5} C_{A} \int \frac{\boldsymbol{\sigma} \times \mathbf{r}}{r}-\frac{1}{\sqrt{ } 10} C_{V} \int \frac{i \mathbf{r}}{r}\right)+\sqrt{\left.\left(\frac{3}{10}\right) C_{V} \int \boldsymbol{\alpha}\right] \operatorname{Re}\left(M_{-1-2}\right)}\right. \\
\left.+\left[\frac{3}{4 \sqrt{ } 5} C_{A} \int \frac{\boldsymbol{\sigma} \times \mathbf{r}}{r}-\frac{3}{\sqrt{ } 10} C_{V} \int \frac{i \mathbf{r}}{r}\right] \operatorname{Re}\left(L_{-2-2}\right)\right\}\end{array}$ \\
\hline 2 & 2 & 2 & $\frac{63}{160} C_{A^{2}}\left(\int i \frac{B_{i j}}{r}\right)^{2} \operatorname{Re}\left(L_{-2-2}\right)$ \\
\hline 3 & 1 & 2 & 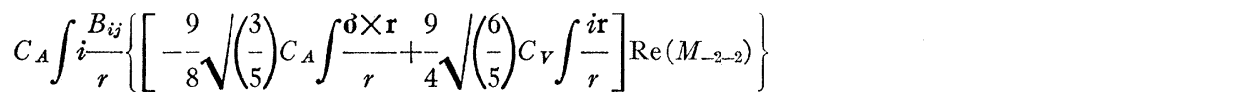 \\
\hline 3 & 2 & 2 & $-\frac{27}{160} C_{A^{2}}\left(\int i \frac{B_{i j}}{r}\right)^{2} \operatorname{Re}\left(M_{-2-2}\right)$ \\
\hline
\end{tabular}

a For $K \neq K^{1}$, we list $b_{J}^{J, 0}\left(K, K^{1} ; J_{f}, J_{i}\right)+b_{J}^{J, 0}\left(K^{1}, K ; J_{f}, J_{i}\right)$.

of the irreducible tensor operators $T_{K L \gamma}$, and this fact is denoted by their dependence on $K$ and $K^{\prime}$. The occurrence of particular particle parameters in particular expressions allows a simple interpretation in terms of coupling schemes which we discuss below.

It is obvious from the formulas given in Appendix IV that the knowledge of the quantities $F$ and $B$ enables one to calculate all the measurable quantities. The numbers $F$ have been given in the literature, our definition of the $F$ 's is related to the usual one (ALD 57) in the following way:

$$
F_{J}{ }^{0, J}\left(K, K^{\prime} ; J_{f}, J_{i}\right)=F_{J}\left(K, K^{\prime} ; J_{f}, J_{i}\right),
$$

where $F_{J}\left(K, K^{\prime} ; J_{f}, J_{i}\right)$ is defined and tabulated in references (ALD 57, FE 55). Furthermore, we have the relation

$$
F_{J}^{J, 0}\left(K, K^{\prime} ; J_{f}, J_{i}\right)=(-)^{K+K^{\prime}} F_{J^{0, J}}\left(K, K^{\prime} ; J_{i}, J_{f}\right)
$$

Therefore, all the quantities $F$ occurring in the expressions in Appendix IV are given in the literature except the quantities $F_{0}{ }^{J} J\left(K, K^{\prime} ; J_{f}, J_{i}\right)$, for which we give a separate table.

The explicit calculation of the particle parameters $B$ is given in Appendixes $\mathrm{V}$ and VI and proceeds as follows (Appendix V): It is a straightforward pro- 
TABLE IV. Values for the functions $L_{V V}$, and $M_{V V}$, in Konopinski-Uhlenbeck approximation [compare (BI 58b)].

We define $\eta=Z W \alpha / k ; \xi=Z \alpha / 2 R ; F(Z, W)=$ Fermi function; $R=$ nuclear radius

$$
S_{m n}=\frac{(m-1) !(n-1) !}{(2 m) !(2 n) !}\left\{1-i \eta\left[\psi_{1}(m)-\psi_{1}(n)\right]\right\} ; \quad \psi_{1}(m)=\frac{\Gamma^{\prime}(m)}{\Gamma(m)} \text { for } m, n \text { positive integer. }
$$

Then, if $m$ and $n$ are positive integers,

$$
\begin{aligned}
& L_{m n}=S_{m n} F(Z, W)(2 k R) l(m)+\imath(n)\left\{\frac{\xi^{2}}{k^{2}}+\left(\frac{m}{2 m+1}+\frac{n}{2 n+1}\right) \frac{\xi}{W}+\frac{m n}{(2 m+1)(2 n+1)}\right. \\
& \left.+\frac{1 Z \alpha}{2 W k}(m-n)\left[\frac{\xi^{2}}{m n}+\left(\frac{1}{(2 m+1) n}+\frac{1}{(2 n+1) m}\right) \xi W+\frac{W^{2}+1}{(2 m+1)(2 n+1)}\right]\right\} \\
& L_{m-n}=L_{-n m}{ }^{*}=S_{m n} F(Z, W)(2 k R) l(m)+\imath(n)\left[2 n \frac{\xi}{W}+\frac{2 m n}{2 m+1}+i \frac{Z \alpha}{k}\left(\frac{m-n}{m} \xi+\frac{m-n}{2 m+1} \frac{p^{2}}{W}-\frac{2 n}{2 m+1} \frac{1}{W}\right)\right] \\
& L_{-m-n}=S_{m n} F(Z, W)(2 k R)^{l(-m)+l(-n)}\left[4 m n+2 i \frac{Z \alpha}{W} k^{2}(m-n)\right] \\
& M_{m n}=S_{m n} F(Z, W)(2 k R) l(m)+l(n) \frac{1}{k W}\left\{\xi^{2}+\left(\frac{m}{2 m+1}+\frac{n}{2 n+1}\right) \xi W\right. \\
& \left.+\frac{m n}{(2 m+1)(2 n+1)} k^{2}+\frac{1 Z \alpha}{2} \frac{i}{k}(m-n)\left[\frac{\xi^{2} W}{m n}+\frac{\left(4 W^{2}-k^{2}\right) \xi}{(2 m+1)(2 n+1)}+\frac{k^{2} W}{(2 m+1)(2 n+1)}\right)\right] \\
& M_{m-n}=M_{-n m^{*}}=S_{m n} F(\dot{Z}, W)(2 k R)^{l(m)+l(-n)}\left[2 n \frac{\xi}{W}+\frac{2 m n}{2 m+1} \frac{k}{W}+i Z \alpha(m-n)\left(\frac{\xi}{m W}+\frac{1}{2 m+1}\right)\right] \\
& M_{-m-n}=S_{m n} F(Z, W)(2 k R)^{l(-m)+l(-n)}[4(m n k / W)+2 i Z \alpha(m-n)]
\end{aligned}
$$

cedure to evaluate the reduced lepton matrix elements and write them in terms of the functions $f_{\kappa}, g_{\kappa}$. Evaluation of the geometrical factors entering into the definition of the $B$ yields $B$ as a bilinear form in integrals over the nucleon coordinates, involving an integration over the functions $f_{k}(r), g_{k}(r)$. The resulting expressions for $B$ are listed in Table III. Here use has been made of the fact, that the reduced matrix elements of the tensor operators $T_{K L \gamma}$ are simply connected to the six nuclear matrix elements listed in Table $\mathrm{I}$. The connection between these two sets of matrix elements is given in Table II.

Since the dependence on energy of all our expressions is contained in the $B$ 's, it is desirable to display this dependence on electron and neutrino energy and momentum explicitly. Therefore, one further step is necessary: One must make a series expansion in powers of $r$ of the functions $f_{\kappa}$ and $g_{\kappa}$, which contain the energy dependence. This is described in detail in Appendix VI, together with corrections to these expansions which are valid for point nuclei. These corrections are due to the finite nuclear size and screening.

The results are collected in Table IV. This table gives all the information needed to calculate measurable quantities in terms of the nuclear matrix elements.
Questions arising in the case $\beta^{+}$emission are treated separately in Appendix VII. ${ }^{3}$

For an interpretation of the particle parameter $B$, it is useful to realize the following angular momentum coupling rules. They may simplify the understanding of the formulas for the spectrum, etc. $K$ and $K^{\prime}$, denote the multipolarity of the two operators, the reduced matrix elements of which are contained in $B . K$ and $K^{\prime}$ have to couple to $J$. Therefore, the maximum possible $J$ is $J_{\max } \leq 2 K_{\max }$, and a glance at Table I shows that $J_{\max } \leq 2$ for allowed, $\leq 4$ for first-forbidden decay. The definition of the $B$ 's in (C 9a), however, also shows that if $j_{e}$ is the total angular momentum carried away by the electron, and $j_{\nu}$ the same quantity for the neutrino, that

$$
\left|j_{e}-j_{\nu}\right| \leq K \leq j_{e}+j_{\nu}, \quad J_{\max } \leq 2 j_{e} .
$$

The first of these relations is obvious, because the two leptons have to carry away the total angular momentum $K$ in the nuclear transition; the second one is a conse-

\footnotetext{
${ }^{3}$ Since experiments on $K$ capture generally do not yield the information on nuclear structure in which we are interested, the appendixes of this paper do not contain formulas for $K$ capture, except for Appendix VII. We refer the reader to the review article by Brysk and Rose (BR 58).
} 
quence of the first and the fact that $K$ and $K^{\prime}$ couple to $J$. Since in allowed transitions $j_{e}$ is limited to $\frac{1}{2}$, we obtain $J \leq 1$ and have, in fact, only to deal with the values $J=0,1$. Similarly in first-forbidden transitions we have $j_{e} \leq \frac{3}{2}$, and therefore $J \leq 3$, which has the consequence that $J=4$ terms do not occur in first, but only in second- and higher-forbidden transitions.

It is now quite obvious which particle parameters are to be expected in the different expressions. In the spectrum shape factor, for example, we have no preferred direction and hence only $J=0$ is possible. Therefore, only such values of $K$ and $K^{\prime}$ will occur that couple to zero; that is, we will have $K=K^{\prime}$. This determines the possible interference terms of nuclear matrix elements that enter into this expression: Only terms of the same multipolarity will interfer. Similarly, for the observation of the $\beta$-circularly polarized $\gamma$ angular correlation, $J$ must be odd (because of parity conservation in the $\gamma$ transition) and has, therefore, the value 1 in allowed and the values 1 and 3 in firstforbidden transitions. $K$ and $K^{\prime}$ must, in an allowed transition, couple to 1 , and therefore we have two types of terms: Interference between Fermi- and GamowTeller matrix elements $\left(K=0, K^{\prime}=1\right)$, and the square of the Gamow-Teller matrix element $\left(K=1=K^{\prime}\right)$.

One can carry through a similar discussion for all cases and in each case see how the formulas can be easily interpreted.

\section{3. $\xi$ APPROXIMATION AND UNIQUE-FORBIDDEN TRANSITION}

As can be seen from Table IV, the general expression for any measurable quantity is quite a complicated function of the neutrino- and electron momenta and energies. There are, however, two limiting cases in which these expressions become fairly simple-the $\xi$ approximation and the unique-forbidden transitions.

The $\xi$ approximation is obtained from the formulas in Table IV by making the assumption $\alpha Z / R \gg W_{0}$, where $W_{0}$ is the maximum total energy of the $\beta$ 's, and has its name from the fact that $\alpha Z / 2 R=\xi$ is a common abbreviation in $\beta$-decay theory. In other words, we assume that the Coulomb energy of the electron at the nuclear radius is larger than its total energy, or that the distortions due to Coulomb forces in the wave function of the electron are much more important than the next term in the expansion of the plane wave, which is of order $k r$. For many decays, this is a reasonable approximation, and implies that terms of order $\alpha Z$ are kept, whereas terms of order $q R$ or $k R$ are to be dropped.

As a consequence all first-forbidden quantities have the same energy and angle dependence as allowed ones. A more precise formulation of the underlying approximation is that the product of $\xi$ with the particular linear combination of matrix elements associated with $\xi$ is large compared to the product of $k$ or $q$ with its particular linear combination of matrix elements. Since, as pointed out in Sec. 1, the nucleon matrix elements which are of order $v / c$ are treated like allowed matrix elements as far as the lepton current is concerned, i.e., electron and neutrino wave function are treated in the limit $r \rightarrow 0$, the $\xi$ approximation has relevance only for the other four matrix elements. These other matrix elements are associated with factors $\xi$, or $q$, or $k$, and only the first factor is being kept.

Since the $\xi$ approximation applies to many firstforbidden $\beta$ decays, and since it has the rather surprising consequence that in this approximation the energy and angle dependence of all quantities is the same as in the allowed case, it might be worth while to elucidate this result somewhat by looking at its causes.

A complete proof, showing that the forementioned statement holds provided that one does not measure the neutrino, is given in Appendix II. Here, we give a brief argument which makes this statement plausible and shows the nature of the $\xi$ approximation.

The assumption $\xi \gg q, k$ implies that we have to replace the neutrino wave function by 1 , as in the allowed case. For the electron wave function, we compare with the allowed case-in the allowed case we had to keep the lowest order terms, that is according to Appendix I:

$$
\begin{aligned}
\psi_{e}^{\mu}=(4 \pi)^{\frac{1}{2}} & \\
\cdot & \left\{\exp \left(-i \Delta_{-1}\right) f_{-1}+(-)^{\mu+\frac{1}{2}} \gamma_{5} g_{1} \exp \left(-i \Delta_{1}\right)\right\} \phi_{-1}^{(\mathrm{I}) \mu} .
\end{aligned}
$$

Here $\phi_{-1}$ has the angular momentum zero, spin $\frac{1}{2}$, and there are two such contributions: $\exp \left(-i \Delta_{-1}\right) f_{-1}$ for the "large" component of the $j=\frac{1}{2}, l=0$ state, and $\exp \left(-i \Delta_{1}\right) g_{1}$ from the "small" component of the $j=\frac{1}{2}$, $l=1$ state. (Small and large components of a Dirac wave function always have opposite parity!)

In the forbidden case, we have (for the four matrix elements in question to which the $\xi$ approximation is applicable) to keep the part of the electron wave function that contains $\mathbf{r}$ in order to get the corresponding matrix elements. From Appendix I, we infer that this part is given by

$$
\begin{gathered}
\psi_{e}^{\mu} \rightarrow(4 \pi)^{\frac{1}{2}}\left\{e^{-i \Delta_{1}} f_{1}(-)^{\mu+\frac{1}{2}}-\gamma_{5} g_{-1} e^{-i \Delta-1}\right\} \phi_{1}{ }^{(\mathrm{I}) \mu} \\
+(8 \pi)^{\frac{1}{2}}\left\{e^{-i \Delta-2} f_{-2}+\gamma_{5}(-)^{\mu+\frac{1}{2}} g_{2} e^{-i \Delta_{2}}\right\} \phi_{-2}(\mathrm{I}) \mu
\end{gathered}
$$

The wave functions with index \pm 1 come from $j=\frac{1}{2}$ states as before, the wave functions with index \pm 2 from $j=\frac{3}{2}$ states. From Appendix I, it also follows that with $r \rightarrow 0$ [neglecting terms of order $(Z \alpha)^{2}$ and higher]

$$
\left.\begin{array}{l}
e^{-i \Delta-1} f_{-1}, e^{-i \Delta_{1}} g_{1} \Rightarrow \text { const } \\
e^{-i \Delta-2} f_{-2}, e^{-i \Delta_{2}} g_{2} \Rightarrow \text { const } \cdot(k r) \\
e^{-i \Delta_{1}} f_{1}, e^{-i \Delta-1} g_{-1} \Rightarrow \text { const } \cdot(Z \alpha)
\end{array}\right\}
$$

where the constants are numbers independent of $Z \alpha$ 
and $r$; and different from zero for $W \neq 1$, where $W$ is the total electron energy.

By using the forms of $\phi_{-1}{ }^{(1) \mu}$ and $\phi_{1}^{(1) \mu}$ given in Appendix II, the part of the electron wave function essential for the allowed decay is given by

$$
\psi_{c}^{\mu}=\text { const } \times(1-\beta) X_{1}^{\mu}
$$

and for the first-forbidden decay in $\xi$ approximation by

$$
\psi_{e}^{\mu}=\operatorname{const} X(-)^{\mu+\frac{1}{2}} i(1-\beta)\left(\mathbf{d} \mathbf{r}_{0}\right)\left(\mathbf{d} \mathbf{k}_{0}\right) X_{2}^{\mu} \cdot(\alpha Z)
$$

where $\mathbf{r}_{0}=\mathbf{r} / r, \mathbf{k}_{0}=\mathbf{k} / k$. The $\mathbf{r}_{0}$ in (3.4b) is the relevant quantity which enters the nuclear matrix element, the usual method being that one writes $r$ instead or $\mathbf{r}_{0}$ and at the same time replaces $(\alpha Z)$ by $\alpha Z / R=2 \xi$, where $R=$ nuclear radius. This procedure is in line with the treatment of the nuclear matrix elements in the general case (see Appendix VI).

Equation (3.4) shows which part of the electron wave function is being kept in $\xi$ approximation, and at the same time makes it plausible that the energy dependence of the resulting expressions is the same one as in the allowed case. Details are given in Appendix II.

As shown there, we obtain the expressions for spectrum shape factor, $\beta-\gamma$ angular correlation, etc., from the allowed case by making the following substitutions for the allowed matrix elements:

$$
\begin{gathered}
-C_{v} \int 1 \rightarrow+C_{A} \int \gamma_{5}+\xi C_{A} \int(\boldsymbol{\sigma} \cdot \mathbf{r}) i^{-1} \\
C_{A} \int \boldsymbol{\sigma} \rightarrow-C_{v} \int \boldsymbol{\alpha}+\xi C_{A} \int \boldsymbol{\sigma} \times \mathbf{r}+\xi C_{v} \int i \mathbf{r} .
\end{gathered}
$$

This is very simple to understand: The scalar matrix element $C_{v} \int 1$ will have to be replaced by a linear combination of scalar matrix elements, $C_{A} \int \gamma_{5}$ and $C_{A} \int(\boldsymbol{\sigma} \cdot \mathbf{r}) i^{-1}$. The factor in front of $C_{A} \int \gamma_{5}$ must be a number independent of $\xi$ (it turns out to be one) since this is a relativistic matrix element, the factor in front of $C_{A} \int i^{-1}(\boldsymbol{\sigma} \cdot \mathbf{r})$ must be of order $\xi$, since this is a nonrelativistic matrix element in the nucleons. Similarly, we can argue for the case of the vector matrix element $\int \boldsymbol{\delta}$. The actual form of the linear combination has, of course, to be worked out in each of the two cases and turns out to be the one given above.

Incidentally, however, it becomes clear why the matrix element $B_{i j}$ does not contribute to the $\xi$ approximation. For the matrix element $B_{i j}$ to occur, electron and neutrino together must carry away at least two units of angular momentum. (Bij has the selection rule $\Delta J=2$.) Therefore, since the $\xi$ approximation assumes the neutrino to be in a $s_{\frac{1}{3}}$ state, the electron must at least be in a $p_{\frac{1}{2}}$ state. However, from (3.3) it is evident that this state does not contribute to the $\xi$ approximation.
In many ways the opposite extreme to the $\xi$ approximation is the "unique forbidden" case, where $\Delta J=2$, $\Delta \pi=-1$, so that only the matrix element $B_{i j}$ contributes. Since this is the only unknown quantity in such a decay, both energy and angular dependence of all the measurable quantities are uniquely determined. Particularly, the spectrum does not have allowed form, since we have just seen that $B_{i j}$ must be accompanied by a factor $k$ or $q$, so that the statistical factor is not the only energy dependent quantity which determines the spectrum as in the case of the $\xi$ approximation and allowed transitions.

All the formulas in Table IV can be easily specified to the case of either the $\xi$ approximation or the uniqueforbidden decay, by neglecting terms of order $k$ and $q$ or eliminating all matrix elements besides $B_{i j}$, respectively.

The considerations of this paragraph have the following important consequence: If the $\xi$ approximation holds exactly, then all the measurable quantities will have the same behavior as in the allowed case, and will depend only on the ratio of two linear combinations of nuclear matrix elements which occur in (3.5). A measurement of the $\beta$ circularly polarized $\gamma$-angular correlation gives, for example, this ratio. This number together with the $\log \mathrm{ft}$ which determines the absolute value of the two linear combinations in (3.5), is all we can ever measure in $\xi$ approximation and is, in fact, not enough information to determine the nuclear matrix elements uniquely. From the point of view of the nuclear physicist, we therefore have to concentrate on those first-forbidden transitions which show deviations from the $\xi$ approximation.

\section{DISCUSSION OF THE MEASURABLE OUANTITIES, SELECTION RULES, AND CANCELLATIONS}

As pointed out in Sec. 3, we can hope to obtain information about the nuclear matrix elements only in the case that the $\xi$ approximation fails. We expect this to happen under several different circumstances:

(1) For light nuclei, where the transition energies are generally large and $Z$ small so that not necessarily is $\xi \gg W_{0}$.

(2) In case that the particular linear combination of matrix elements which is multiplied with the leading term in the $\xi$ approximation happens to be small, i.e., if the matrix elements cancel each other.

(3) If the matrix elements in front of the leading term all by themselves are very small, because an intrinsic nuclear selection rule (which goes beyond the simple selection rule for the total spin) inhibits the matrix element. Such a rule could be derived from the shell, or collective model. In such a case, it may turn out that the matrix element $B_{i j}$ plays an important role, and we expect significant changes in the energyand angular dependence of various measurable quantities.

The causes discussed in (1) and (2) have essentially 
the same effect: The leading term in the $\xi$ approximation is not the only one to consider; we have to take into account the next term, but the $B_{i j}$ terms are still small. In these two cases, which we consider together, we talk about a "cancellation effect" (KOT 59b). On the other hand, we describe the phenomenon discussed under (3) as the "selection rule effect."

In the following, we discuss how the matrix elements enter into the various expressions, how deviations from the $\xi$-approximation such as discussed above influence those expressions, and how selection rules of the type (3) occur in nuclei.

\section{A. Spectrum of the $\beta$ Particles}

As shown in Appendix II, in $\xi$ approximation, the spectrum has the allowed form, i.e., it is determined by the phase-space factors of the leptons. In general, the shape correction factor-that is, the spectrum divided by the phase-space factor-has the following form (Konopinski-Uhlenbeck approximation):

$$
C(W)=k\left(1+a W+b W^{-1}+c W^{2}\right) .
$$

Here, $a, b$, and $c$ vanish in the $\xi$ approximation. In fact, $k$ is of order $\xi^{2}$, ak and $b k$ are of order $\xi$, and $c k$ is of $\operatorname{order}^{4} \xi^{0} . a$ and $b$ are independent of $W$. If the cancellation effect is predominant, we should therefore expect $a$ and $b$ to play a significant role, whereas for the selection rule effect, $c$ is the most important quantity (it contains terms of order $B_{i j}{ }^{2}$ ).

In practice, a good analysis of the spectrum turns out to be very difficult. In particular, one often deals with complex spectra where there are less energetic decays occurring to one or more higher excited levels so that one cannot measure the spectrum very precisely except in the vicinity of the end point energy. Also, an inspection of (4.1) shows that over a small range of energies it is extremely hard to obtain good values for $a, b$, and $c$ from an analysis of the experimental data, even if they have small errors, because the effects of $a, b$, and $c$ partly tend to cancel each other so that one can construct a fairly straight line for $C(W)$ even for relatively large values of $a, b$, and $c . \mathrm{Au}^{198}$ (SI 58 , SI 60, ST $60 \mathrm{~b}$ ) is a good example of this behavior.

It is also interesting that a unique-forbidden shape factor is not necessarily an indication for an unique forbidden transition, as was pointed out by Kotani (KOT 59b).

\section{B. Log ft Value}

This number may give some indication as to whether we have a deviation from the $\xi$ approximation: Whereas for the case of the $\xi$ approximation the $\log \mathrm{ft}$ values group around 6.0, significantly larger values may hint on a deviation.

${ }^{4}$ This symbol $k$ should not be confused with the electron momentum $k$ as used in the previous section.

\section{Longitudinal Polarization of the Electrons}

In $\xi$ approximation, this polarization turns out to be given by $\mp v / c$, as in the allowed case where the plus or minus sign is determined by the charge of the $\beta$ particle. In case the selection rule effect holds, we would still expect the polarization to be given by this expression because also in $B_{i j}$ approximation it is $\mp v / c$. For the cancelation effect, which presumably is responsible for the peculiar $R a E$ decay (see, e.g., GE $59 \mathrm{a}$, GE $59 \mathrm{~b})$, we expect a deviation from the $\mp v / c$ rule, which has indeed been found experimentally (BU 58a, Bu 58b, WE 58).

\section{Electron Distribution from Oriented Nuclei}

For first-forbidden transitions, this distribution function has the form

$$
W(\theta)=a+b P_{1}(\cos \theta)+c P_{2}(\cos \theta)+d P_{3}(\cos \theta) .
$$

$a$ and $b$ are of order $\xi^{2}$, but involve also terms of order $\xi^{1}$ and $\xi^{0} . c$ is of order $\xi^{1}$ and contains terms of order $\xi^{0}$, and $d$ comes solely from the $B_{i j}$ approximation and is of order $\xi^{0} \times p^{3} / W$. In the $\xi$ approximation, one should therefore expect only $a$ and $b$ to be present. If a selection rule effect occurs, then one should look for terms in $\cos ^{2} \theta$ and $\cos ^{3} \theta$ (if these terms are possibly existent, which still depends on the nuclear spins involved in the transition, the coefficients $b, c$, and $d$ depend also on geometrical factors), whereas a cancellation effect should only give terms up to order $\cos ^{2} \theta$. If a precise measurement of this angular distribution as a function of electron energy could be performed, it would yield much information about nuclear matrix elements. On the other hand, the information obtainable with this experiment is equivalent to the information which we obtain using the $\beta-\gamma$ angular correlation and circular polarization correlation function.

\section{E. Electron-Gamma Correlation Functions}

The $\beta-\gamma$ angular correlation function has the form

$$
W_{1}\left(\theta_{\beta \gamma}\right)=1+\epsilon P_{2}\left(\cos \theta_{\beta \gamma}\right),
$$

where $\epsilon$ contains a factor $p^{2} / W$ and is of order $\xi^{-1}$. Therefore, the angular correlation is isotropic in $\xi$ approximation, and a measurement of $W$ which yields a nonzero $\epsilon$ is a strong indication of a failure of the $\xi$ approximation.

Except for geometrical coefficients, $\epsilon$ in Eq. (4.3) is equal to $c / a$ in Eq. (4.2). It has the following form:

$$
\epsilon=\frac{k}{C(W)} \frac{p^{2}}{W}(R+\epsilon \cdot W)
$$

where $R$ is of order $\xi^{1}$, and $e$ is of order $\xi^{0}$. In the $B_{i j}$ approximation, $\epsilon$ is negative and has a unique energy dependence. However, fairly small deviations from a pure $B_{i j}$ approximation may change $\epsilon$ considerably, so that it may have either positive or negative values. It 
may also be negative in the case of the cancellation effect. In view of the complicated energy dependence of $\epsilon$, ( $R$ and $e$ are energy independent), ${ }^{5}$ a measurement of $\epsilon$ alone will not suffice to give enough information about the nuclear matrix elements, unless it is accompanied by a measurement of the $\beta-\gamma$-circular polarization correlation function. This has the following form:

$$
W_{2}\left(\theta_{\beta \gamma} ; \tau\right)=\omega \cdot \tau \cdot \cos \theta_{\beta \gamma} \cdot(p / W),
$$

where $\tau=+1$ for right-, $\tau=-1$ for left-handed circularly polarized light, and $\omega$ is of the form:

$$
\omega=\frac{k}{C(W)} \frac{f+g W+h W^{2}+l p^{2} P_{2}\left(\theta_{\beta \gamma}\right)}{W_{1}\left(\theta_{\beta \gamma}\right)} .
$$

Since we have discussed $k, C(W)$, and $W_{1}\left(\theta_{\beta \gamma}\right)$ before, we only need to consider $f, g, h$, and $l$ as functions of $\xi ; f$ is of order $\xi^{2}, g$ of order $\xi$, and $h$ and $l$ are of order $\xi^{0}$. In the $\xi$ approximation $\omega$ is, therefore, constant with respect to both angle and energy. In allowed transitions, a determination of $\omega$ measures the ratio of Fermi and Gamow-Teller matrix element. Similarly, in forbidden transitions with $\xi$ approximation, a measurement of $\omega$ determines the ratio of two linear combinations of forbidden matrix elements, the one which, according to Sec. 3 and Appendix II replaces $\int 1$, and the one which replaces $\int \delta$ in the formulas for allowed transitions. Since $\int 1$ is replaced by a certain linear combination of matrix elements of scalar operators $(\Delta J=0)$, and similarly $\int \boldsymbol{\alpha}$ by a certain combination of matrix elements of vector operators $(\Delta J= \pm 1,0)$ for forbidden transitions with $\Delta J= \pm 1, \omega$ has the value it is expected to have in allowed transitions for $\int 1=0$, namely $\pm \frac{1}{3}$, where the sign is equal to the charge of the emitted $\beta$ particle.

In case of the cancellation effect, we expect mainly $g$ to come into play, whereas in case of the $B_{i j}$ approximation $h$ and $l$ are important. Therefore, for the selection rule effect we should expect $\omega$ to have a stronger angular dependence than in the cancellation effect. On the other hand, Kotani (KOT 59b) has pointed out that it depends on the spin change of the transition and, therefore, on the geometrical factors entering into $g$, $h$, and $l$, and the combination of matrix elements possibly contributing to them, whether $\omega$ for either cancellation or selection rule effect depends more strongly on angle or energy.

\section{F. Longitudinally Polarized $\beta-\gamma$ Angular Correlation Function}

In coincidence with the $\gamma$ ray, the longitudinal polarization of the $\beta$ 's becomes angular dependent and has the form

$$
P_{\text {long }}\left(\theta_{\beta \gamma}\right)=P_{\text {long }}{ }^{(0)}+\alpha P_{2}\left(\cos \theta_{\beta \gamma}\right) .
$$

Here, $\alpha$ contains terms of order $\xi^{1}$ and $\xi^{0}$, the first of

${ }^{5}$ This statement holds true except for Coulomb corrections. which is essentially given by $\left(W / p^{2}\right) \epsilon$ in Eq. (4.4). The term $P_{\text {long }}{ }^{(0)}$ is the expression for the longitudinal polarization without measuring the $\gamma$ ray; it turns out, that the term $\alpha P_{2}\left(\cos \theta_{\beta \gamma}\right)$, (where $\alpha$ itself is angular dependent) changes the polarization in the most cases presumably only by a few per cent.

\section{G. Transversely Polarized $\beta-\gamma$ Angular Correlation Function}

This function, measured either in or perpendicular to the $\beta-\gamma$ plane, provides more information than the longitudinal polarization, because it is zero in both the $\xi$ and the $B_{i j}$ approximation. However, the degree of polarization to be expected is for most cases not very large, in units of $v / c$ it will seldom amount to more than 10 or $20 \%$.

\section{THEORETICAL ESTIMATES OF THE NUCLEAR MATRIX ELEMENTS; SELECTION RULES AS CONSEQUENCES OF VARIOUS NUCLEAR MODELS}

Two different approaches have been made in order to estimate the values of the six nuclear matrix elements occurring in the first-forbidden $\beta$ decay. Some authors (e.g., AH 52a, AH 52b; AH 53, PU 51, and PE 53) establish relations between nuclear matrix elements as a consequence of commutation laws with the nuclear Hamiltonian. Another line of thought (AH 52a, HE $54, \mathrm{KON} 54$, RO 54a, RO 54b) tries to reduce the matrix elements to their nonrelativistic form, and then applies a nuclear model in order to evaluate them. In this approach, from nuclear models we obtain selection rules more specific than the general selection rules deduced from the behavior of the operators under rotation and the parity operation.

In the first approach, one tries to find a relation between matrix elements with the same transformation properties; that is, one tries to calculate the constants $A$ and $B$ in the relations

$$
\begin{aligned}
\int \boldsymbol{\alpha} & =A \int i \mathbf{r} \\
\int \gamma_{5} & =B \int i^{-1}(\boldsymbol{\sigma} \cdot \mathbf{r}) .
\end{aligned}
$$

Relations of this form must obviously hold for these four matrix elements. Values of $A$ and $B$ are derived in the following way.

The total nuclear Hamiltonian has the three parts

$$
H=\sum_{i} H_{\text {Dirac }}(i)+H_{\text {Coul }}+H_{\text {inter }},
$$

where $H_{\text {Dirac }}(i)$ is the free particle Dirac-Hamiltonian for particle $i, H_{\text {Coul }}$ the Coulomb interaction and $H_{\text {inter }}$ the nucleon-nucleon non-Coulomb interaction. Let us now consider the operator $\tau_{k} \mathbf{r}_{k}$, for example $\left(\tau_{k}\right.$ is the plus or minus component of the isotopic spin operator):

$$
\begin{aligned}
&\left\langle f\left|\left(W_{f}-W_{i}\right) \mathbf{r}_{k} \tau_{k}\right| i\right\rangle=\left\langle f\left|\left[H, \mathbf{r}_{k} \tau_{k}\right]\right| i\right\rangle \\
&=\left\langle f\left|\left[H_{\text {Dirac }}(k), \mathbf{r}_{k} \tau_{k}\right]\right| i\right\rangle+\left\langle f\left|\left[H_{\text {Coul }}, \mathbf{r}_{k} \tau_{k}\right]\right| i\right\rangle \\
&+\left\langle f\left|\left[H_{\text {inter }}, \mathbf{r}_{k} \tau_{k}\right]\right| i\right\rangle .
\end{aligned}
$$


We know, that $\left[H_{\text {Dirac }}(k), \mathbf{r}_{k} \tau_{k}\right]=i \boldsymbol{\alpha}_{k} \tau_{k}$. The two other commutators are evaluated in the following way:

$$
\begin{aligned}
\left\langle f\left|\left[H_{\text {Coul }}, \mathbf{r}_{k} \tau_{k}\right]\right| i\right\rangle=\sum_{n}\left\{\left\langle f\left|H_{\text {Coul }}\right| n\right\rangle\left\langle n\left|\mathbf{r}_{k} \tau_{k}\right| i\right\rangle\right. \\
\left.-\left\langle f\left|\mathbf{r}_{k} \tau_{k}\right| n\right\rangle\left\langle n H_{\text {Coul }} \mid i\right\rangle\right\} \\
\approx\left\{\left\langle f\left|H_{\text {Coul }}\right| f\right\rangle-\left\langle i\left|H_{\text {Coul }}\right| i\right\rangle\right\}\left\langle f\left|\mathbf{r}_{k} \tau_{k}\right| i\right\rangle
\end{aligned}
$$

and similarly for $H_{\text {inter }}$. It seems justified to neglect nondiagonal terms of the Coulomb interaction [this was tested by Ahrens \& Feenberg (AH 52a) using determinantal wave functions], and also of the interaction Hamiltonian, the argument being that $\left\langle f\left|\mathbf{r}_{k} \tau_{k}\right| n\right\rangle$ connects the state $|f\rangle$ with states $|n\rangle$ which are not too different from $|i\rangle$ (because $\left\langle f\left|\mathbf{r}_{k} \tau_{k}\right| i\right\rangle$ is possible). The validity of the shell model shows, however, that $\left\langle n\left|H_{\text {inter }}\right| i\right\rangle$ must be small. Finally, $\left\{\left\langle f\left|H_{\text {Coul }}\right| f\right\rangle-\left\langle i\left|H_{\text {Coul }}\right| i\right\rangle\right\}$ can be calculated from the Coulomb energy difference, and $\left\{\left\langle f\left|H_{\text {inter }}\right| f\right\rangle-\right.$ $\left.\left\langle i\left|H_{\text {inter }}\right| i\right\rangle\right\}$ from the symmetry energy. This way, one arrives at the following relations:

$$
\frac{\Lambda \alpha Z}{2 R} \int i \mathbf{r}=\int \boldsymbol{\alpha} ; \frac{\Lambda \alpha Z}{2 R} \int \frac{1}{i}(\boldsymbol{d r})=-\int \gamma_{5},
$$

where $R$ is the nuclear radius, $Z$ the charge, and

$$
\Lambda=\left(1+\frac{W_{i}-W_{f}}{m c^{2}} \frac{A^{\frac{1}{3}}}{Z}\right)
$$

[this result is taken from AH 52a; Pursey (PU 51) obtains a slightly different result using a different nuclear Hamiltonian, and so do Rose and Osborn (RO 54a)]. Measurements of the spectrum of $\operatorname{Pr}^{144}$ (see Sec. 6) give the ratio $\int \gamma_{5} / \int(\boldsymbol{\sigma} \cdot \mathbf{r}) / i$, which seems to agree with these estimates in order of magnitude (PE $60)$.

The other approach reduces the "relativistic" matrix elements $\int \gamma_{5}$ and $\int \boldsymbol{\alpha}$ to their nonrelativistic form and then applies nuclear models to calculate all of the six matrix elements. The reduction is most simply done by a Foldy-Wouthuysen transformation and gives in lowest order in $1 / M$ where $M$ is the nucleon mass (RO 54a)

$$
\int \alpha \rightarrow-(1 / M) \int \mathrm{p}, \int \gamma_{5} \rightarrow-(1 / M) \int(\boldsymbol{\alpha} \cdot \mathrm{p})
$$

(see also HE 54, KON 54, AH 52a). For the pseudoscalar interaction, compare Sec. $6 .^{6}$ From the result of the transformation, it is clear that the "relativistic" matrix elements deserve the name "momentum type" matrix elements in contrast to the "coordinate type" matrix elements $\int i \mathbf{r}, \int(\boldsymbol{\sigma} \cdot \mathbf{r}) / i, \int[\mathbf{\sigma} \times \mathbf{r}]$ and $\int B_{i j}$. This form of the matrix elements is now subject to selection rules derived from specific nuclear models. If one adopts the nuclear shell model, then various reasons can inhibit

\footnotetext{
${ }^{6}$ In case one considers the influence of third-order terms on first-order forbidden $\beta$ decay the usual replacement of the relativistic operators may lead into difficulties. See (RO 54a).
}

the $\beta$ transitions (BR 51, BR 53, KI 54, RO 54b, TA 51 , TA 54a, TA 54b):

1. The "coefficient of fractional parentage" between the two states involved in the transition may be very small, because we have either strong configurational mixing in one of the states, or the two core wave functions (that is, the wave functions for the whole nucleus but the last particle) have a small overlap for another reason. King and Peaslee (KI 54) find that these "unfavored" transitions have matrix elements, which on the average are a factor of three smaller than the ones for "favored" transitions.

2 . The $\beta$-decaying nucleon makes a transition from a state $j$ to a state $j^{\prime}$ and $\Delta j=\left|j-j^{\prime}\right|>\Delta I=\left|I_{i}-I_{f}\right|$, where $I_{i}$ and $I_{f}$ are the initial and final spins of the nucleus. In this case, the single-particle matrix elements may be inhibited. If, for example, $2 \geq \Delta j \geq \frac{3}{2}$, and $\Delta j>\Delta I$, then the matrix element $B_{i j}$ will not be inhibited whereas the others are. This may lead to the "selection rule" effect. King and Peaslee (KI 54) report that for $\Delta I=\Delta j$, the nuclear matrix elements have the same order of magnitude no matter whether $\Delta j=0,1$, or 2. However, in cases where $\Delta I<\Delta j$ the squares of the matrix elements are reduced by a factor of about ten. This effect is often referred to as " $j$ forbiddenness".

Alga et al. (AL 55a, AL 55b, AL 57a, AL 57b, DA 60 , VO 57) study the selection rules which are a consequence of the collective and Nilsson model. For nuclei with $155<A<185$ and $A>225$, the level spectrum consists of rotational bands, which for even-even nuclei follow the spin sequence $I=0,2,4, \cdots$ for the lowest band, $I=K, K+1, \cdots$ for the higher bands, where $K$ is an intrinsic quantum number and integer, and for odd $A$ nuclei have bands of the form $I=K, K+I, \cdots$ where $K$ is half-integer. If $L$ is the multipolarity of the $\beta$-decay operator, then the additional selection rule

$$
\left|K_{i}-K_{f}\right|=\Delta K \leq L
$$

holds (" $K$ forbiddenness"). Since $K$ is not a very good quantum number except for large deformations, there will only be a retardation of the transitions with $L<\Delta K$. This may also lead to the "selection rule" effect. For $\beta$ transitions leading to states of the same rotational band for which the intrinsic wave function is the same to a very good approximation, one obtains the branching ratio for a given multipolarity as a simple ratio of Clebsch-Gordan coefficients (AL 55b). In the simple case that either $K_{i}$ or $K_{f}$ is zero this ratio reads

$$
\frac{B\left(L, I_{i} \rightarrow I_{f}\right)}{B\left(L, I_{i} \longrightarrow I_{f}^{\prime}\right)}=\frac{C^{2}\left(I_{i} L I_{f} ; K_{i} K_{f}-K_{i}\right)}{C^{2}\left(I_{i} L I_{f}^{\prime} ; K_{i} K_{f}-K_{i}\right)}
$$

and has a slightly more complicated form for $K_{i} \neq 0$, $K_{f} \leftarrow 0$, and $L \geq K_{i}+K_{f}$. Davydov (DA 60) considers the extension of these selection rules to the case of non axially symmetrical nuclei.

Alaga et al. (AL 55a, AL 57a, AL 57c) give selection rules for single-particle transitions in a deformed nuclear 
potential (Nilsson model); they consider only the case of large deformations and give the selection rules for the various $\beta$-decay operators in terms of the asymptotic quantum numbers of the Nilsson scheme.

\section{PSEUDOSCALAR INTERACTION}

The first-forbidden decay seems to offer the best possibility to detect the pseudoscalar interaction or to put upper limits on its existence. Although throughout this paper, we neglect this interaction, we here summarize the present evidence against its existence (AL $57 \mathrm{~b}, \mathrm{BH} 60$, GE 58a, GE 58b, TA 60). Since in the $\mathrm{O}^{-} \rightarrow \mathrm{O}^{+}$transitions like the $\mathrm{Pr}^{144}$ and $\mathrm{Ho}^{166}$ decay only the two axial vector matrix elements $\int \gamma_{5}$ and $\int(1 / i)(\boldsymbol{d} \cdot \mathbf{r})$ can contribute besides a possible pseudoscalar interaction, it is these nuclei that have been the object of a search for this interaction. The only experimental data obtainable are the $\log \mathrm{ft}$, the spectrum, and the longitudinal electron polarization. One tries to fit them with two parameters, $\int(1 / i) \boldsymbol{\sigma} \cdot \mathbf{r}$ and $\lambda=$ $\int \gamma_{5} / \int(1 / i) \boldsymbol{\sigma} \cdot \mathbf{r}$, e.g., and any significant failure of the fit indicates the presence of a pseudoscalar term.

The only well-established experimental data on $0^{-} \rightarrow 0^{+}$transitions are the ones on $\operatorname{Pr}^{144}$ (CO 60, FR 57, GE 58, GR 58, HE 58, ME 60) and on $\mathrm{Ho}^{166}$ (BU 59 , CO 58). The data on $\operatorname{Pr}^{144}$ have been analyzed by Tadic (TA 60); he did not use finite-size corrections for the electron wave functions and obtained an inconclusive result. Both the $\mathrm{Ho}^{166}$ and $\mathrm{Pr}^{144}$ data have been analyzed independently by Bhalla (BH 60 ), who has made use of the Foldy-Wouthuysen transformation in order to obtain the proper reduction of the pseudoscalar interaction (this was also done by TA 60), and calculated finite deBroglie wavelength and finite nuclear size effects with a digital computer. In this paper, which uses the two component theory of the neutrino, an attempt is made to obtain upper limits on the pseudoscalar interaction as a function of $\lambda$, the ratio of the axial vector matrix elements, and it is concluded that the absence of the pseudoscalar interaction is in agreement with existing experimental data; for $-200 \leq$ $\lambda \leq 200,\left|C_{P} / C_{A}\right| \leq 90$, where $C_{P}$ is the pseudoscalar coupling constant.

\section{SPECIAL CASES: $\mathrm{Sb}^{124}, \mathrm{Au}^{198}, \mathrm{Bi}^{210}(\mathrm{RaE})$}

As demonstrations of the possibility of learning something about the nuclear matrix elements in beta-decay, we consider the well-investigated decays of $\mathrm{Sb}^{124}, \mathrm{Au}^{198}$, and $\mathrm{Bi}^{210}$. These are (besides the $0^{-} \rightarrow 0^{+}$decays described in the previous section) the only first-forbidden decays that have been fully analyzed so far.

\section{1. $\mathrm{Sb}^{124}$ (HA 60, KOT 59b, MO 59, ST 52, ST 60, YA $52 b$ )}

This nucleus decays via a $3^{-}(\beta) 2^{+}(\gamma) 0^{+}$transition, the maximum energy of the beta rays is $W_{0}=5.6$, and there is another beta decay with an end-point energy of $W_{0}=4.1$, which obscures measurements at low energies. The log-ft value is unusually large, $\log \mathrm{ft}=$ 10.6 , and the spectrum shows deviations from the allowed form. The $\beta-\gamma$ correlation shows a large asymmetry $(|\epsilon| \leq 40 \%)$. By a combination of the measurements of the log-ft value, the $\beta-\gamma$ angular correlation and the $\beta$ circularly polarized $\gamma$-angular correlation, it has been possible to determine the value of the four nuclear matrix elements which are important in this decay: $\int i \mathbf{r}, \int i B_{i j}, \int \boldsymbol{\alpha}, \int(\boldsymbol{\sigma} \times \mathbf{r})$. In particular, the $\beta-\gamma$ angular correlation shows that there is no $P_{4}$ term, which supports the view that this is a first forbidden decay. The values of the matrix elements are the following ones: $\hbar=c=m=1, R=$ nuclear radius)

$$
\begin{gathered}
\left|\int i B_{i j}\right| / R=(1.20 \pm .15) \times 10^{-2} \\
\left|\int i \mathbf{r}\right| / R=(1.2 \pm 1.2) \times 10^{-3} \\
\left|\int(\boldsymbol{\delta} \times \mathbf{r})\right| / R=(0.1 \pm 0.4) \times 10^{-3} \\
\left|\int \boldsymbol{\alpha}\right|=(3.1 \pm 2.4) \times 10^{-4} \\
\int \boldsymbol{\alpha} / \int i B_{i j}<0 .
\end{gathered}
$$

Clearly, this nucleus shows a "selection rule" effect, and no "cancellation effect." The "coordinate type" matrix elements of multipolarity 1 are strongly suppressed. The same is true for the matrix element $\int \boldsymbol{\alpha}$, whereas $(1 / R) \int i B_{i j}$ dominates the other three matrix elements. In a "normal" situation, one would expect the coordinate-type matrix elements to be of order one, and $(1 / R) \int i B_{i j}$ and $\int \boldsymbol{\alpha}$ to be of order 0.1 . The $\mathrm{Sb}^{124}$ nucleus is a very fortunate example for two reasons: the beta decay involves a spin change of one unit. There are, therefore, only four relevant matrix elements, and the deviations from the $\xi$ approximation are very large; larger, in fact, than the average deviation from the $\xi$ approximation for the nuclei listed by Kotani (KOT $59 \mathrm{~b})$.

\section{2. $\mathrm{Au}^{198}$ (GE 58, SI 58, SI 60, ST 60b)}

Ninety-nine percent of the beta decay proceeds from the ground state of $\mathrm{Au}^{198}\left(2^{-}\right)$to the first-excited state $\left(2^{+}\right)$of $\mathrm{Hg}^{198}$ with an end-point energy of $960 \mathrm{kev}$. The $\log \mathrm{ft}$ of the transition is $\log \mathrm{ft}=7.46$, and the subsequent E2 transition to the ground state of $\mathrm{Hg}^{198}\left(0^{+}\right)$ has an energy of $611 \mathrm{kev.}$

The $\xi$ value for $A u^{198}$ is $\xi \cong 16$, so that (except for a cancellation or selection rule effect) the $\xi$ approximation should describe the situation very satisfactorily. We 
expect an error of $1 / \xi \cong 6 \%$ (The $\log \mathrm{ft}$ is fairly small.) This is indeed the case.

The spectrum has statistical shape within $\pm 5 \%$; the $\beta$-circularly polarized $\gamma$-angular correlation coefficient is proportional to $p / W$, as expected, and the $\beta-\gamma$ angular correlation coefficient which is approximately $3 \%$ at the end-point energy is explicable by the Coulomb corrections to the $\xi$ approximation. (Here small terms of order $1 / \xi$ come into play and produce the anisotropy).

Also a measurement of the two transverse polarizations of the electrons in coincidence with the $\gamma$ rays is in accord with the $\xi$ approximation.

This implies that in this nucleus, it is not possible to determine nuclear matrix elements separately. On the other hand, the reported measurements did establish the decay scheme and the validity of the $\xi$ approximation.

\section{3. $\operatorname{RaE}\left(\mathrm{Bi}^{210}\right)$. (ALI 59, BAN 60, BI 58a, BU 58a, BU 58b, GEI 58, GE 59a, GE 59b, LEW 57a, NA 56, NE 59, PL 59, WE 58, WU 53, YA 53c)}

RaE decays from its $1^{-}$state to the $0^{+}$ground state of $\mathrm{Po}^{210}$. The spectrum is known to show deviations from the allowed form, and the polarization has been found to be larger than $-v / c$ and to have a peculiar energy dependence.

Since we are dealing with a $\Delta I=1$ yes transition, the following matrix elements can contribute: $\int \boldsymbol{\alpha}, \int i \mathbf{r}$, $\int \boldsymbol{\sigma} \times \mathbf{r}$. This is true only if we neglect third-order and finite nuclear-size corrections (see Appendix VI). Most of the analyses of the spectrum and polarization (the only measurable quantities besides the $\log \mathrm{ft}$ ) have been made on this basis. If one takes the finite nuclear size into account, five different matrix elements might contribute and the inclusion of third order terms might increase this number even more.

The end point energy of the electron is given by $W_{0}=3.3$, and $\xi \approx 15$, so that, similar to the case of $\mathrm{Au}^{198}$, one might expect the $\xi$ approximation to hold. This is not the case, as the experiments show, and it is the cancellation effect, which is responsible for this fact.

An analysis of the data shows that one can fit the spectrum and the measured values of the polarization by just two parameters,

$$
\xi_{1}=\frac{C_{v} \int \text { ir }}{C_{A} \int \boldsymbol{\delta} \times \mathbf{r}} \text { and } \eta_{1}=\frac{1}{\xi} \frac{C_{v} \int \alpha}{C_{A} \int \boldsymbol{\delta} \times \mathbf{r}} .
$$

This analysis yields for $\xi_{1}$ the best value $\xi_{1}=0.3$, and for the "leading term" in the $\xi$ approximation the quantity

$$
x=\xi \cdot \eta_{1}-2 \xi \frac{1+\xi_{1}}{1+S}
$$

where $S=\left[1-(Z \alpha)^{2}\right]^{3}$, the value $x=3.3$. This shows indeed that a cancellation effect takes place. Newby et al. (NE 59) and Banerjee and Zeh (BAN 60) have discussed this situation on the basis of the shell model and obtained a satisfactory understanding of the data.

This nucleus is also suited for a test whether invariance under time reversal holds (LEW 57); the corresponding experiments (WE 59) are not in disagreement with this invariance requirement.

\section{A $2^{-}(\beta) 2^{+}(\gamma) 0^{+}$TRANSITION AS ILLUSTRATION}

The preceding chapters and the appendixes contain the pertinent formulas for $\beta$ decay and their discussion. It is felt, however, that an elucidation of the content of the previous chapters by means of an example might be instructive. In this chapter, we give a number of curves for spectrum shape factor, $\beta-\gamma$ angular correlation coefficient, and $\beta$ circularly polarized $\gamma$-angular correlation coefficient calculated for various choices of the six nuclear matrix elements, for a transition of the type $2^{-}(\beta) 2^{+}(\gamma) 0^{+}$, and for an end-point $\beta$ energy $W_{0}=2.5$. This choice of parameters corresponds approximately to the $\beta$ decay of $\mathrm{Rb}^{86}$. It is not the purpose of these figures, however, to help in an analysis of the $\beta$ decay of this nucleus. Instead, they are to demonstrate the dependence of the experimental quantities mentioned above on the nuclear matrix elements.

Such a demonstration seems advisable for various reasons: It becomes obvious that, generally speaking, the measurable quantities depend strongly on these matrix elements in the sense that they are very sensitive to a change of even one of them. The graphs show, on the other hand, that the measurement of one of the quantities alone can not determine the nuclear matrix elements uniquely. The curves might also be of help for the experimentalist who wants to decide at which angles he should measure a correlation function, how precise the measurement should be, and how accurately he should determine the energy dependence of the correlation coefficient, for example.

In the use of these figures a certain caution is required because they are typical only for 2-2-0 transitions. Usually the form of any of the curves changes little if one changes the end-point energy; this is not true if one changes the decay scheme. Therefore, one should avoid general conclusions about the behavior of the functions displayed. The actual form of the curves also depends on the charge $Z$ of the nucleus under consideration.

The curves are calculated in the KonopinskiUhlenbeck approximation, for two different sets of choices of nuclear matrix elements: (a) Deviations from $\xi$ approximation. Here, we assume throughout that $\int i B_{i j}=0$, and that a certain cancellation effect takes place (Figs. 1-8). (b) Deviations from $B_{i j}$ approximation. Here, the largest matrix element is the 


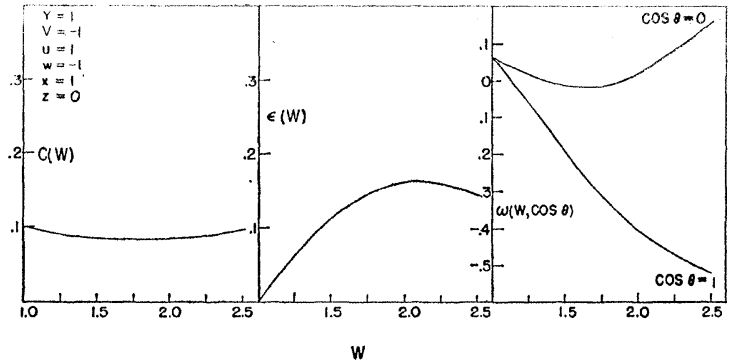

FIG. 1.

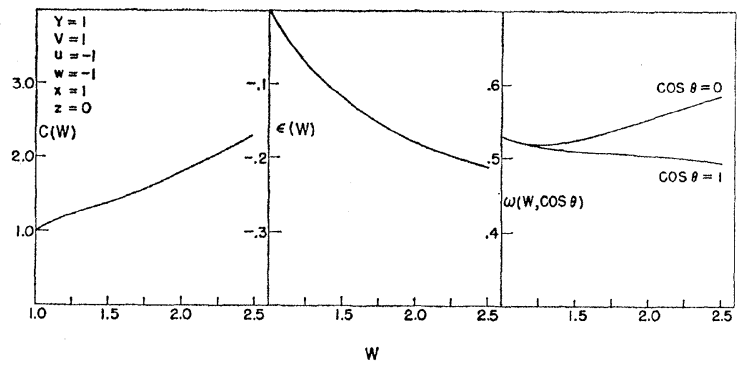

FIG. 2.

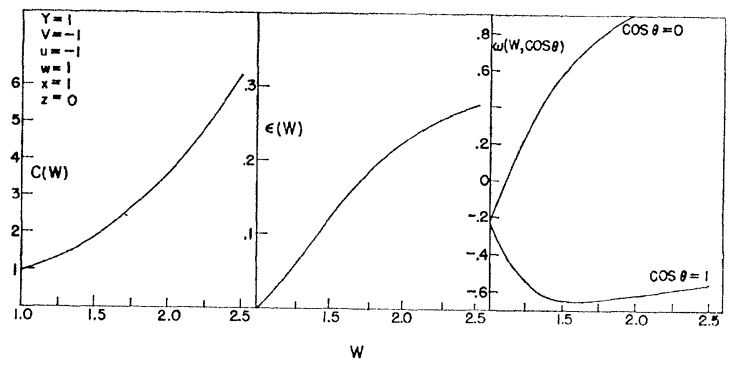

FIG. 3.

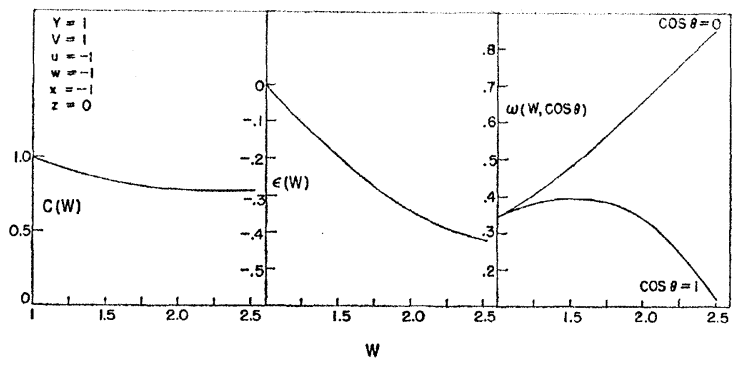

FIG. 4.

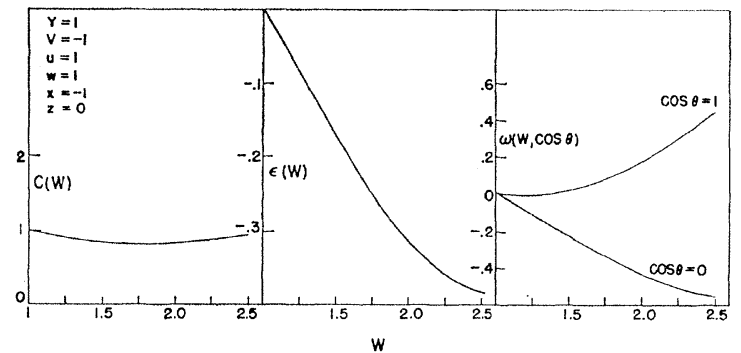

FIG. 5.

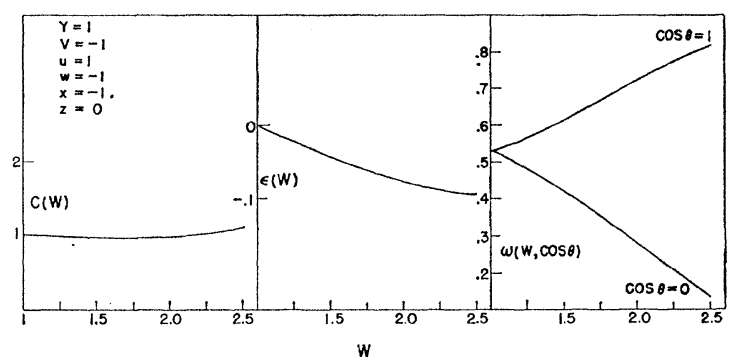

FIG. 6.

FIGs. 1 to 12 . The spectrum shape factor $C(W)$, the $\beta-\gamma$ angular correlation anisotropy coefficient $\epsilon(W)$, and the $\beta$ circularly polarized $\gamma$-correlation coefficient $\omega(W, \cos \theta)$ for a 2-2-0 transition. $W$ is the electron energy in units $\hbar=m=c=1$, and $\theta$ the angle between electron and $\gamma$ ray. The twelve figures show the three functions for various choices of nuclear matrix elements $V, Y, x, u, w$, and $z$ as defined in this section. The value of the nonvanishing nuclear matrix elements is indicated in the upper left-hand corner of each figure.

matrix element $\int i B_{i j}$, and we vary the values for the two matrix elements leading in the $\xi$ approximation. The other three matrix elements are assumed to be negligible (Figs. 9-12).

Each figure contains three curves: The spectrum shape factor $C(W)$ normalized so that $C(1)=1$, the coefficient of the $\beta-\gamma$ angular correlation function $\epsilon(W)$ and the coefficient $\omega$ of the $\beta$ circularly polarized $\gamma$ angular correlation function. $\omega(W, \cos \theta)$ is a function of both energy and angle; the curves show $\omega(W$, $\cos \theta=0)$ and $\omega(W, \cos \theta=1)$ as functions of energy. The quantities $C(W), \epsilon(W)$ and $\omega(W, \cos \theta)$ have been defined in Sec. 4.

At the upper left-hand side, each figure contains a list of equations. These equations define the particular set of parameters for which the figure was calculated. The meaning of the symbols used in these figures is the following (KOT 59b) :

$$
\begin{aligned}
& V=C_{A} \int \gamma_{5}+\xi C_{A} \int(\boldsymbol{\sigma} \cdot \mathbf{r}) / i \\
& Y=C_{V} \int \boldsymbol{\alpha}-\xi C_{A} \int[\boldsymbol{\sigma} \times \mathbf{r}]-\xi C_{V} \int i \mathbf{r} \\
& x=+C_{V} \int i \mathbf{r} \\
& u=+C_{A} \int[\mathbf{\sigma} \times \mathbf{r}] \\
& w=+C_{A} \int(\boldsymbol{\sigma} \cdot \mathbf{r}) / i \\
& z=-C_{A} \int i B_{i j}
\end{aligned}
$$




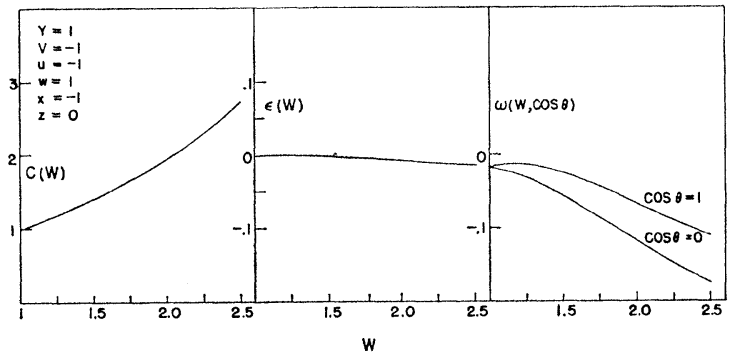

FIG. 7.

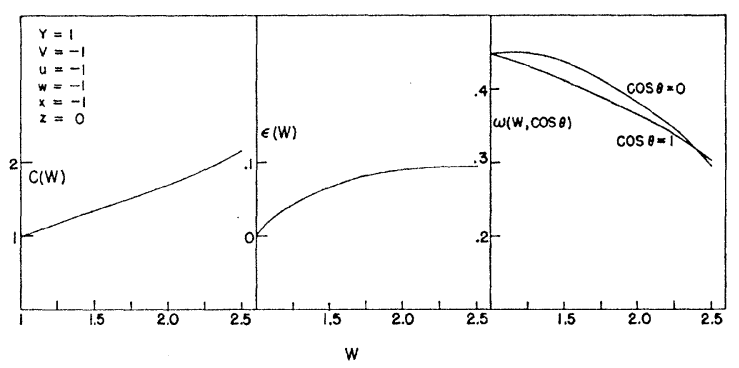

FIG. 8.

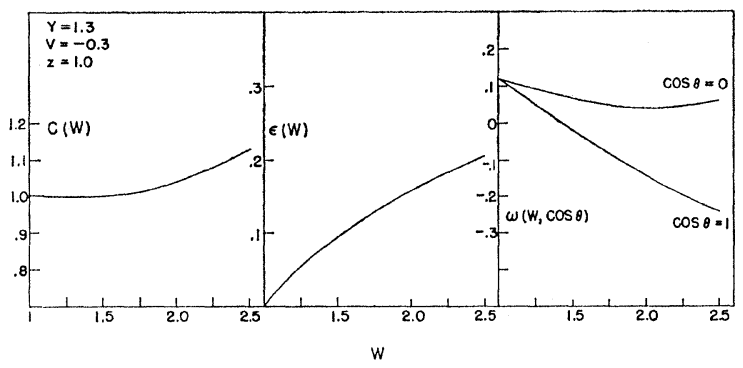

FIG. 9.

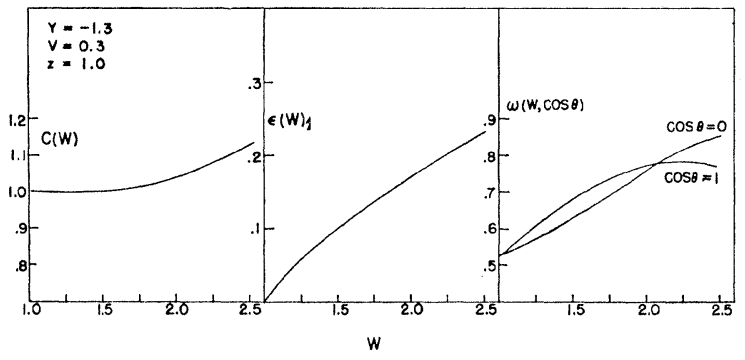

FIg. 10.

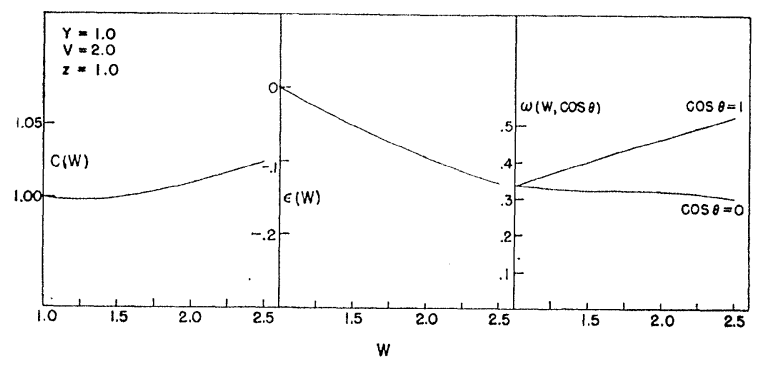

FIG. 11.

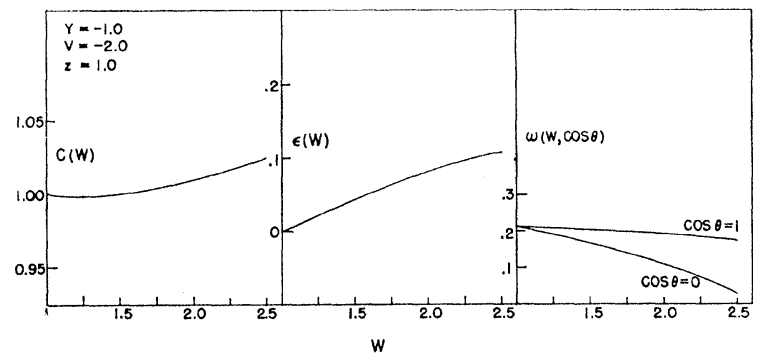

FIG. 12.

(caption on opposite page.)

\section{ACKNOWLEDGMENTS}

The author expresses his deep gratitude to Professor F. Boehm, without whose permanent encouragement and interest this paper would have never been completed, and who carefully read the manuscript.

For the treatment of the $\beta$-decay density matrix in Appendixes I and II, he owes thanks to Professor J. H. D. Jensen (University of Heidelberg) for many remarks and comments. He wants to acknowledge the kind hospitality of the California Institute of Technology.

\section{APPENDIX I. RELATIVISTIC COULOMB WAVE FUNCTIONS}

In this appendix we describe the construction of a solution of Dirac's equation for the case of the continuous spectrum. Although the results are well known (YO 59) we feel that because of the different notations used in the literature a consistent representation of the results may be useful. The method of derivation employed here, which does not make use of a particular representation of the Dirac matrices, gives a good insight into the nature of these solutions and a better understanding of the well-known fact to be proven in Appendix II, that in $\xi$ approximation first-forbidden and allowed $\beta$ decay give the same answers.

We first construct the spin eigenfunction [eigenfunction of the operator $\left.\chi_{\mathrm{op}}=\beta(\boldsymbol{\sigma} \cdot \mathrm{L}+1)\right] \quad$ (Part 1), then by means of the Dirac equation its radial dependence (Part 2), and from the asymptotic conditions the linear combination which is equivalent to a plane wave in the field-free case (Part 3).

\section{Construction of Spin Eigenfunctions}

We define the usual operators

$$
\begin{gathered}
\mathbf{L}=[\mathbf{r} \times \mathbf{p}], \mathbf{p}=(1 / i) \nabla, \\
\chi_{\mathrm{op}}=\beta(\boldsymbol{\sigma} \cdot \mathbf{L}+1),
\end{gathered}
$$

where $\beta^{2}=1, \sigma^{2}=3,[\beta, \boldsymbol{\sigma}]=0$. (For the definition of the Dirac matrices, see Appendix VIII.) Since $\alpha=$ $+\gamma_{5} \boldsymbol{\delta}, \gamma_{5}^{2}=1$, and $\left[\gamma_{5}, \beta\right]=2 \gamma_{5} \beta$, we see that $\chi_{\mathrm{op}}$ commutes with $(\boldsymbol{\alpha} \cdot \mathbf{p})$ and therefore with the Dirac- 
Hamiltonian for any central potential. Since furthermore

$$
\chi_{\mathrm{op}}^{2}=\left(j_{\mathrm{op}}^{2}\right)+\frac{1}{4},
$$

we infer that $\chi_{\text {op }}$ has the eigenvalues $\chi= \pm 1, \pm 2$, $\pm 3, \cdots$, and that $\chi^{2}=\left(j+\frac{1}{2}\right)^{2}$, where $j(j+1)$ is eigenvalue to $j_{\text {op }}{ }^{2}=\left(\mathbf{L}+\frac{1}{2} \mathbf{\sigma}\right)^{2}$.

We want to construct functions $\phi_{\chi}{ }^{\mu}$ which are defined by

$$
\left.\begin{array}{c}
\chi_{\mathrm{op}} \phi_{\chi}{ }^{\mu}=+\chi \phi_{\chi}{ }^{\mu} \\
j_{z} \phi_{\chi}{ }^{\mu}=\mu \phi_{\chi}{ }^{\mu} \cdot
\end{array}\right\}
$$

We decompose the problem by defining

$$
\begin{aligned}
\phi_{\chi}{ }^{(\mathrm{I}) \mu} & =\frac{1}{2}(1-\beta) \phi_{\chi}{ }^{\mu}, \\
\phi_{\chi}{ }^{(\mathrm{II}) \mu} & =\frac{1}{2}(1+\beta) \phi_{\chi}{ }^{\mu} .
\end{aligned}
$$

In the representation

$$
\beta=\left(\begin{array}{rr}
-1 & 0 \\
0 & 1
\end{array}\right)
$$

this corresponds to picking out the "upper" and "lower" component of the wave function. Since $(1-\beta) \cdot(1+\beta)=0$, Eq. (A4) yields, using Eq. (A2) :

$$
\left.\begin{array}{r}
(\boldsymbol{\sigma} \cdot \mathbf{L}+1) \phi_{\chi}{ }^{(\mathrm{I}) \mu}=-\chi \phi_{\chi}{ }^{(\mathrm{I}) \mu} ;(1+\beta) \phi_{\chi}{ }^{(\mathrm{I}) \mu}=0, \\
(\boldsymbol{\sigma} \cdot \mathbf{L}+1) \phi_{\chi}{ }^{(\mathrm{II}) \mu}=\chi \phi_{\chi}{ }^{(\mathrm{II}) \mu} ;(1-\beta) \phi_{\chi}{ }^{(\mathrm{II}) \mu}=0 .
\end{array}\right\}
$$

Since $\left[\left(\boldsymbol{\alpha} \cdot \mathbf{r}_{0}\right), \chi_{\text {op }}\right]=0$, where $\mathbf{r}_{0}=\mathbf{r} /|\boldsymbol{r}|$, we know that if $\phi_{\chi}^{(I) \mu}$ obeys Eq. (A7), then

$$
\phi_{\chi}^{(\mathrm{II}) \mu}=+i\left(\boldsymbol{\alpha} \cdot \mathbf{r}_{0}\right) \phi_{\chi}^{(\mathrm{I}) \mu}
$$

also fulfills Eq. (A7). By confining attention for the moment to the special case $\mu= \pm \frac{1}{2}$, it is easy to see that

$$
\phi_{\chi}{ }^{(\mathrm{I}) \mu}=\frac{1}{2}(1-\beta)(\boldsymbol{d} \cdot \mathbf{L}-\chi) Y_{l}{ }^{0}\left(\mathbf{k}_{0}, \mathbf{r}_{0}\right) X_{\frac{1}{2}}{ }^{\mu}
$$

fulfills Eq. (A7), if we choose $\chi=l$ or $\chi=-l-1$. Here, $\mathbf{k}_{0}$ is an arbitrary unit vector, and $X_{\frac{1}{2}}{ }^{\mu}$ is defined by the equations

$$
\begin{aligned}
\sigma_{z} X_{\frac{1}{2}}{ }^{\mu} & =2 \mu X_{\frac{1}{2}}{ }^{\mu}, \\
\sigma^{2} \cdot X_{\frac{1}{2}}{ }^{\mu} & =3 X_{\frac{1}{2}}{ }^{\mu} .
\end{aligned}
$$

$Y_{l}{ }^{0}\left(\mathbf{k}_{0}, \mathbf{r}_{0}\right)$ is a usual spherical harmonic (see Appendix VIII) with $\mathbf{k}_{0}$ as the $z$ axis. For the two possible choices for $\chi$ one obtains $(\chi \neq 0)$ :

$$
\begin{aligned}
& \text { for } \quad \chi=l: \quad j=l-\frac{1}{2} \quad \chi=j+\frac{1}{2} \text {, } \\
& \chi=-l-1: \quad j=l+\frac{1}{2} \quad \chi=-\left(j+\frac{1}{2}\right) .
\end{aligned}
$$

We normalize $\phi_{\chi}^{(\mathrm{I}) \mu}$ by defining

$$
\begin{aligned}
\phi_{\chi}(\mathrm{I}) \mu=(-\operatorname{Sg} \chi)^{\mu-\frac{1}{2} \frac{1}{2}}(1-\beta) & \\
& \frac{(\boldsymbol{\sigma} \cdot \mathbf{L})-\chi}{[|x|(2 l+1)]^{\frac{1}{2}}} i^{l} Y_{l^{0}}\left(\mathbf{k}_{0}, \mathbf{r}_{0}\right) X_{\frac{1}{2}}^{\mu} .
\end{aligned}
$$

This is convenient, because in this definition $\phi_{\chi}^{(\mathrm{I}) \mu}$ is for $\mu= \pm \frac{1}{2}$ identical with

$\phi_{\chi}^{(\mathrm{I}) \mu}=\frac{1}{2}(1-\beta) \sum_{\tau} c\left(l \frac{1}{2} j ; \tau, \mu-\tau\right) Y_{l^{\tau}} X_{\frac{1}{2}}^{\mu-\tau} \cdot i^{l}$,

if we choose $j$ and $l$ according to Eq. (A9) and the $z$ axis to be given by the direction of $\mathbf{k}_{0}$.

It is easy to show that $(\operatorname{Sg} \chi \equiv \operatorname{sign} \chi) i\left(\boldsymbol{\delta} \mathbf{r}_{0}\right) \phi_{\chi}{ }^{\mu}=$ Sg $\chi \phi_{-\chi}{ }^{\mu}$ and that therefore a correspondingly reasonable choice for the normalization of $\phi_{\chi}^{(\mathrm{II}) \mu}$ is given by Eq. (A8).

Finally we note that any linear combination of the form

$$
\psi_{\chi}^{\mu}=\left(f_{\chi}+i g_{\chi}\left(\boldsymbol{\alpha} \cdot \mathbf{r}_{0}\right)\right) \phi_{\chi}^{(\mathrm{I}) \mu}
$$

is eigenfunction to $\chi_{\mathrm{op}}$ with eigenvalue $\chi, f_{\chi}$, and $g_{\chi}$ being arbitrary.

\section{Determination of the Radial Wave Functions}

We now use the Dirac equation (see Appendix VIII) to determine the quantities $f_{\chi}$ and $g_{\chi}$ as functions of $r$. We write this equation for a central potential $V(r)$ in the form (we use $\hbar=c=m=1$ throughout)

$$
[+(\boldsymbol{\alpha} \cdot \mathbf{p})+\beta+E-V(\boldsymbol{r})] \psi_{\chi}^{\mu}(\boldsymbol{r})=0
$$

$\psi_{\chi}^{\mu}(r)$ is eigenfunction to $\chi_{\mathrm{op}}$ and therefore given by Eq. (A11).

We use the identity

$(1 / i r)(\boldsymbol{\sigma} \cdot \mathbf{L})\left(\boldsymbol{\alpha} \cdot \mathbf{r}_{0}\right)$

$$
=-(2 / i r)\left(\boldsymbol{\alpha} \cdot \mathbf{r}_{0}\right)-(1 / r)(\mathbf{r} \cdot \mathbf{p})\left(\boldsymbol{\alpha} \cdot \mathbf{r}_{0}\right)+(\boldsymbol{\alpha} \cdot \mathbf{p}) .
$$

Inserting Eq. (A11) into Eq. (A12), we obtain

$$
\begin{aligned}
((1 / i \boldsymbol{r})[(\boldsymbol{\sigma} \cdot \mathbf{L}) & +2+i(\mathbf{r} \cdot \mathbf{p})] i g_{\chi}(r)+[\beta+E-V(r)] f_{\chi}(r) \\
+ & \left\{(1 / i r)[\boldsymbol{\sigma} \cdot \mathbf{L}+2+i(\mathbf{r} \cdot \mathbf{p})] f_{\chi}(\boldsymbol{r})\right. \\
& \left.\left.+i[\beta+E-V(r)] g_{\chi}(r)\right\}\left(\boldsymbol{\alpha} \cdot \mathbf{r}_{0}\right)\right) \phi_{\chi}(\mathrm{I}) \mu=0,
\end{aligned}
$$

or by multiplying with $\frac{1}{2}(1+\beta)$ and $\frac{1}{2}(1-\beta)$, using Eq. (A7) :

$\left(\frac{d}{d r}-\left(\frac{\chi-1}{r}\right)\right) g_{\chi}(r)+(E-V(r)-1) f_{\chi}(r)=0$,

$\left(-\frac{d}{d r}-\frac{\chi+1}{r}\right) f_{\chi}(r)+(E-V(r)+1) g_{\chi}(r)=0$.

Upon introducing the radial wave functions $F_{\chi}(r)=$ $r f_{\chi}(r), G_{\chi}(r)=r g_{\chi}(r)$, we have

$$
\left.\begin{array}{l}
\frac{d}{d r} F_{\chi}(r)=-\frac{\chi}{r} F_{\chi}+(E+1-V) G_{\chi}, \\
\frac{d}{d r} G_{\chi}(r)=-(E-1-V) F_{\chi}+\frac{\chi_{G}}{r} .
\end{array}\right\}
$$

By Eq. (A11) and the solutions of Eqs. (A14) which are integrable at the origin, our solution $\psi_{\chi}{ }^{\mu}$ is defined for every $\chi$ and $\mu= \pm \frac{1}{2}$. 
In the particular case of a Coulomb field, the functions $f_{\chi}(r)$ and $g_{x}(r)$ are given by (YO 59)

$$
\begin{aligned}
& f_{\chi}=[(W+1) / 2 W]^{\frac{1}{2}}(k r)^{\gamma-1} C(\gamma, \eta) \operatorname{Re}\left(\lambda_{\chi}\right), \\
& g_{\chi}=-[(W-1) / 2 W]^{\frac{1}{2}}(k r)^{\gamma-1} C(\gamma, \eta) \operatorname{Im}\left(\lambda_{\chi}\right) .
\end{aligned}
$$

Here the units $\hbar=m=c=1$ have been used, $k^{2}=W^{2}-1$, $W$ is the energy of the particle, and

$$
\begin{gathered}
\gamma=+\left[\chi^{2}-(Z \alpha)^{2}\right]^{\frac{1}{2}}, \\
\eta=(\alpha Z W / k)>0 \text { for electrons, } \\
\lambda_{\chi}=(\gamma+i \eta) \exp [i(\varphi-k r)] \\
\cdot{ }_{1} F_{1}(\gamma+1+i \eta, 2 \gamma+1 ; 2 i k r), \\
e^{2 i \varphi}=e^{-\pi i}\{[\chi-i(Z \alpha / k)] /(\gamma+i \eta)\},-\pi<\varphi \leq 0, \\
C(\gamma, \eta)=2^{\gamma} e^{\pi \eta / 2}[|\Gamma(\gamma+i \eta)| / \Gamma(2 \gamma+1)], \\
\alpha=e^{2} / \hbar c=e^{2} .
\end{gathered}
$$

The Coulomb potential is given in the form $V(r)=$ $-Z e^{2} / r$ and the solutions given here correspond to positive energies (negatons). In case of positrons, one has to replace $Z$ by $-Z$. The negative energy negaton wave function can be obtained from the positive energy solution for a repulsive Coulomb interaction (positron wave function) by charge conjugation:

$$
\psi_{-E}(Z)=i \alpha_{2} \beta \psi_{+E} *(-Z)
$$

The asymptotic behavior of $f_{\chi}(r)$ and $g_{\chi}(r)$ is given by $f_{\chi}(r) \Rightarrow[(W+1) / 2 W]^{\frac{1}{2}} j_{l(\chi)}\left(k r+\eta \ln 2 k r+\Delta_{\chi}\right)$,

$$
g_{\chi}(r) \Rightarrow \operatorname{Sg} \chi[(W-1) / 2 W]^{\frac{1}{2}} j_{l(x)}\left(k r+\eta \ln 2 k r+\Delta_{\chi}\right)
$$

where

$$
\begin{aligned}
\Delta_{\chi}=\varphi+(\pi / 2)[1-\gamma & +l(\chi)] \\
& -\operatorname{Arg}[\Gamma(\gamma+i \eta)]=\delta_{\chi}+(\pi / 2) l(\chi)
\end{aligned}
$$

is the relativistic Coulomb phase shift.

\section{Determination of the Plane-Wave Solution}

We have to form such a linear combination

$$
\psi^{\mu}=\sum_{\chi \neq 0} C_{\chi} \psi_{\chi}^{\mu}
$$

that $\psi^{\mu}$ becomes asymptotically equal to a plane wave plus incoming (or outgoing) spherical waves. ${ }^{7}$ In order to do this we expand a plane wave solution $\left(\mathbf{k}_{0}=\mathbf{k} /|k|\right.$,

\footnotetext{
${ }^{7}$ Because of the logarithmic radial dependence of (A15b), this cannot be fulfilled. This has been discussed by Gordon (GO 28). In the following we take the logarithmic terms formally into account. See also Appendix VI.
}

$u_{\frac{1}{2}}^{\mu}=$ spinor solution of Dirac's equation)

$$
\begin{aligned}
& {[(W+1) / 2 W]^{\frac{1}{3}} \cdot \exp (i \mathbf{k} \cdot \mathbf{r}) u_{\frac{1}{2}}^{\mu}=\exp (i \mathbf{k} \cdot \mathbf{r})} \\
& \cdot\left\{[(W+1) / 2 W]^{\frac{1}{2}}-\left(\boldsymbol{\alpha} \cdot \mathbf{k}_{0}\right)[(W-1) / 2 W]^{\frac{1}{2}}\right\} \\
& \cdot \frac{1}{2}(1-\beta) X_{\frac{1}{3}}{ }^{\mu}
\end{aligned}
$$

into eigenfunctions $\phi_{\chi}{ }^{\mu}$. Since for the comparison we need only the "large" components, we multiply the plane wave solution first by $\frac{1}{2}(1-\beta)$ :

$$
\begin{aligned}
& \frac{1}{2}(1-\beta)[(W+1) / 2 W]^{\frac{1}{2}} \exp (i \mathbf{k} \cdot \mathbf{r}) X_{\frac{1}{2}}{ }^{\mu} \\
& =[(W+1) / 2 W]^{\frac{1}{2}} \sum_{l=0}^{\infty}[4 \pi(2 l+1)]^{\frac{1}{2}} i^{l} j_{l}(k r) Y_{l}^{0} \\
& \left(\mathbf{k}_{0}, \mathbf{r}_{0}\right) \frac{1}{2}(1-\beta) X_{\frac{1}{2}}{ }^{\mu} \\
& =[(W+1) / 2 W]^{\frac{1}{2}} \sum_{l=0}^{\infty}[4 \pi|\chi|]^{\frac{1}{2}} i^{l} j_{l}(k r) \\
& \frac{l-\boldsymbol{d} \cdot \mathbf{L}+l+1+\boldsymbol{o} \cdot \mathbf{L}}{[(2 l+1)|\chi|]^{\frac{1}{2}}} \frac{1}{2}(1-\beta) Y_{l}{ }^{0}\left(\mathbf{k}_{0}, \mathbf{r}_{0}\right) X_{\frac{1}{2}}^{\mu} \\
& =[(W+1) / 2 W]^{\frac{1}{2}} \sum_{\chi \neq 0}[4 \pi|\chi|]^{\frac{1}{2}} j_{l(\chi)}(k r) \\
& (-\operatorname{Sg} \chi)^{\mu+\frac{1}{2}} \phi_{\chi}^{(\mathrm{I}) \mu} .
\end{aligned}
$$

Multiplying Eq. (A16) by $\frac{1}{2}(1-\beta)$ and taking the asymptotic value, one obtains [we assume that the $\mathbf{k}_{0}$ occurring in Eq. (A16) is identical to the unit vector $\mathbf{k}_{0}=\mathbf{k} /|k|$ in Eq. (A17)]

$$
[(W+1) / 2 W]^{\frac{1}{2}} \sum_{\chi \neq 0} C_{\chi} j_{l(x)}\left(k r+\eta \ln 2 k r+\Delta_{\chi}\right) \phi_{\chi}^{(\mathrm{I}) \mu} \text {. }
$$

In case we want to construct a solution which behaves asymptotically like a plane wave plus incoming scattered waves, we have to equate the outgoing parts of Eqs. (A17) and (A18) [after multiplying Eq. (A17) by $\exp ( \pm i \eta \ln 2 k r)]$

$$
C^{\text {in }}=[4 \pi|\chi|]^{\frac{1}{2}}(-\operatorname{Sg} \chi)^{\mu+\frac{1}{2}} \exp \left(-i \Delta_{\chi}\right), \quad \text { (A19a) }
$$

otherwise the ingoing parts [after multiplying Eq. (A17) by $\exp (-i \eta \ln 2 k r)]$

$$
C_{\chi}^{\text {out }}=[4 \pi|\chi|]^{\frac{1}{2}}(-\operatorname{Sg} \chi)^{\mu+\frac{1}{2}} \exp \left(i \Delta_{\chi}\right)=C_{\chi}{ }^{\text {in } *} \text {. }
$$

The two solutions which we obtain are therefore

$$
\begin{aligned}
\psi^{( \pm) \mu}=\sum_{\chi \neq 0}[4 \pi|\chi|]^{\frac{1}{2}}( & -\operatorname{Sg} \chi)^{\mu+\frac{1}{2}} \\
\cdot & \exp \left( \pm i \Delta_{\chi}\right)\left[f_{\chi}+i g_{\chi}\left(\boldsymbol{\alpha} \cdot \mathbf{r}_{0}\right)\right] \phi_{\chi}^{(\mathrm{I}) \mu},
\end{aligned}
$$

where the plus or minus sign stands for out- or ingoing scattered waves. Observing that

$$
\left[f_{\chi}+i(\boldsymbol{\alpha} \cdot \mathbf{r}) g_{\chi}\right] \phi_{\chi}{ }^{(\mathbf{I}) \mu}=\psi_{\chi}{ }^{\mu} \text { and that }
$$

$$
c\left(l(\chi), \frac{1}{2}, j(\chi) ; o, \mu\right)=(-\operatorname{Sg} \chi)^{\mu+\frac{1}{2}}\{|\chi| /[2 l(\chi)+1]\}^{\frac{1}{2}}
$$

we obtain the equivalent representation

$$
\begin{array}{r}
\psi^{( \pm) \mu}=\sum_{\chi \neq 0}\{4 \pi[2 l(\chi)+1]\}^{\frac{1}{2}} c\left(l(\chi) \frac{1}{2} j(\chi) ; o, \mu\right) \\
\cdot \exp \left( \pm i \Delta_{\chi}\right) \psi_{\chi}^{\mu} .
\end{array}
$$


TABLE V. How to replace the matrix elements $\int \sigma, \int 1$ in "allowed" expressions, if one wants to write down the corresponding expression for a first-forbidden transition in $\xi$ approximation.

\begin{tabular}{cccc}
\hline \hline & Allowed & 1st-forbidden terms of order $v / c$ & $\begin{array}{c}\text { 1st-forbidden terms of order } \xi \text { according } \\
\text { to }(\mathrm{B} 10)\end{array}$ \\
\hline$A_{i}$ & $-i C_{A} \int \boldsymbol{\sigma}$ & $i C_{V} \int \boldsymbol{\alpha}$ & $+C_{V} \int \mathbf{r}-i C_{A} \int(\boldsymbol{\sigma} \times \mathbf{r})$ \\
$A_{4}$ & $C_{V} \int 1$ & $-C_{A} \int \gamma_{5}$ & $+i C_{A} \int\left(\boldsymbol{\sigma} \cdot \mathbf{r}_{0}\right)$
\end{tabular}

Therefore the rule holds

$$
\begin{aligned}
-C_{V} \int 1 & \rightarrow C_{A} \int \gamma_{5}+\xi C_{A} \int \frac{1}{i}(\boldsymbol{\sigma} \cdot \mathbf{r}) \\
& C_{A} \int \boldsymbol{\sigma} \rightarrow-C_{V} \int \boldsymbol{\alpha}+\xi C_{V} \int i \mathbf{r}+\xi C_{A} \int(\boldsymbol{\sigma} \times \mathbf{r})
\end{aligned}
$$

\section{APPENDIX II. DENSITY MATRIX IN COULOMB FIELD}

The method discussed in this section is related to (JA 59) and (DE 59). The density matrix (or Casimir operator) is defined by

$$
\rho^{( \pm)}\left(\mathbf{r}, s ; \mathbf{r}^{\prime}, s^{\prime}\right)=\sum_{\mu} \psi^{( \pm) \mu}(\mathbf{r}, s) \psi^{*( \pm) \mu}\left(\mathbf{r}^{\prime}, s^{\prime}\right)
$$

Here, $\psi^{*}$ denotes the hermitian conjugate, and $\mathbf{r}$ and $s$ are space and spin variables. Clearly the knowledge of $\rho^{( \pm)}$is sufficient to calculate all the quantities of interest in $\beta$ decay.

From Appendix I, we now write the general form of the density matrix in the Coulomb (or any central) field. Starting from this form, we derive the density matrices pertinent to allowed and first-forbidden $\beta$ decay. The latter one is specialized to the so-called $\xi$ approximation, and it is shown that all experiments have a dependence on the nuclear matrix elements for first-forbidden $\beta$ decay, which can be obtained directly from the formulas for allowed decay by substituting for every allowed matrix element a certain linear combination of first-forbidden ones.

We define the operator

$$
\begin{aligned}
O_{\chi}^{ \pm} & =(-\operatorname{Sg} \chi) \exp \left( \pm i \Delta_{\chi}\right)\left[f_{\chi}(\boldsymbol{r})+i g_{\chi}(\boldsymbol{r}) \cdot\left(\boldsymbol{\alpha} \cdot \mathbf{r}_{0}\right)\right] \\
& \cdot\{4 \pi /[2 l(\chi)+1]\}^{\frac{1}{2}} \imath^{l(\chi)}(\boldsymbol{\alpha} \cdot \mathbf{L}-\chi) Y_{l}\left(\mathbf{k}_{0}, \mathbf{r}_{0}\right) \frac{1}{2}(1-\beta) .
\end{aligned}
$$

According to Eqs. (B1) and (A20), $\rho$ is then given by

$$
\rho^{( \pm)}=\sum_{\chi, \chi^{\prime} \neq 0} O_{\chi}^{( \pm)}(\mathbf{r}) O_{\chi^{\prime}}{ }^{*( \pm)}\left(\mathbf{r}^{\prime}\right)
$$

since $\sum_{\mu} X_{\frac{1}{2}}^{\mu} X_{\frac{1}{2}} *_{\mu}=1$.

In the case of allowed $\beta$ decay, neither electron nor neutrino carry away any angular momentum. It is furthermore assumed, that $k R \ll 1$ and $q R \ll 1$, where $q$ is the neutrino momentum and $R$ the nuclear radius. This approximation replaces the plane wave for the neutrino by one, and for the electron wave function equations Eq. (A15) show that the only remaining terms in Eq. (B2) are the ones with $\chi= \pm 1$. We have in this approximation

$$
f_{ \pm 1}=[(W+1) / 2 W]^{\frac{1}{2}}(k r)^{\gamma-1} C(\gamma, \eta) \operatorname{Re}\left(\lambda_{ \pm 1}\right),
$$$$
g_{ \pm 1}=-[(W-1) / 2 W]^{\frac{1}{2}}(k r)^{\gamma-1} C(\gamma, \eta) \operatorname{Im}\left(\lambda_{ \pm 1}\right) \text {, }
$$

where

$$
\lambda_{ \pm 1}=(\gamma+i \eta) \exp \left(i_{\varphi_{ \pm 1}}\right), \gamma=\left[1-(Z \alpha)^{2}\right]^{\frac{1}{2}} .
$$

In this case, we also have from Eqs. (A8) and (A10)

$$
\begin{aligned}
& \phi_{-1}(\mathrm{I}) \mu=(4 \pi)^{-\frac{1}{2} \frac{1}{2}}(1-\beta) X_{\frac{1}{2}}{ }^{\mu} \text {, } \\
& \phi_{+1}(\mathrm{I}) \mu=(-)^{\mu+\frac{1}{2}} \cdot i \cdot(4 \pi)^{-\frac{\lambda}{2} \frac{1}{2}}(1-\beta)\left(\boldsymbol{\sigma} \cdot \mathbf{r}_{0}\right)\left(\boldsymbol{\sigma} \cdot \mathbf{k}_{0}\right) X_{\frac{1}{2}}{ }^{\mu} \text {, } \\
& \phi_{1}{ }^{(\mathrm{II}) \mu}=i\left(\boldsymbol{\alpha} \cdot \mathbf{r}_{0}\right) \phi_{1}^{(\mathrm{I}) \mu}=(-)^{\mu-\frac{1}{2}}(4 \pi)^{-\frac{1}{2}} \\
& \cdot \frac{\gamma_{5}}{2}(1-\beta)\left(\boldsymbol{d} \cdot \mathbf{k}_{0}\right) X_{\frac{1}{2}}^{\mu},
\end{aligned}
$$$$
\phi_{-1}(\mathrm{II}) \mu=i\left(\boldsymbol{\alpha} \cdot \mathbf{r}_{0}\right) \phi_{-1}(\mathrm{I}) \mu=i /(4 \pi)^{\frac{1}{2}} \frac{\gamma_{5}}{2}(1-\beta)\left(\boldsymbol{\sigma} \cdot \mathbf{r}_{0}\right) X_{\frac{1}{2}}{ }^{\mu}
$$

The lepton matrix element $\left(\psi_{\epsilon}\left|O_{\mu}\right| \psi_{v}\right)$, is a spacedependent function which has to be inserted as a transition operator between the nuclear states. Since we have approximated the neutrino wave by 1 , and since the operator $O_{\mu}$ is space independent, the space dependence of this matrix element stems only from $\psi_{e}$. Equation (B5) shows that we have two types of orbital dependence - terms independent of $\boldsymbol{r}_{0}$ and terms linear in $\mathbf{r}_{0}$. Since the latter terms would require a change of parity of the nuclear states, we discard these terms in an allowed transition (they become important in a forbidden transition), and keep only $\phi_{1}{ }^{(\mathrm{II}) \mu}$, and $\phi_{-1}^{(\mathrm{I}) \mu}$. This way, we obtain for $\rho^{( \pm)}: \rho^{( \pm)}$allowed= $O^{( \pm)} \cdot O^{( \pm)} *$, where

$O^{ \pm}=\left\{\exp \left( \pm i \Delta_{-1}\right) \cdot f_{-1}-\exp \left( \pm i \Delta_{1}\right) g_{1}\left(\boldsymbol{\sigma} \cdot \mathbf{k}_{0}\right) \gamma_{5}\right\} \frac{1}{2}(1-\beta)$.

Using Eqs. (A15), a straightforward calculation shows, that for a Coulomb field (according to Appendix VI, we put $r=r^{\prime}=R$, the nuclear radius):

$$
\rho_{\text {allowed }}^{( \pm)}=\frac{1}{2} F(Z, E)\left\{1-\frac{\beta \gamma}{W}-\frac{\boldsymbol{\alpha} \cdot \mathbf{k}}{W} \pm i \beta \frac{\alpha Z}{k} \cdot \frac{(\boldsymbol{\alpha} \cdot \mathbf{k})}{W}\right\} .
$$


Here we have defined the Fermi function by

$$
F(Z, E)=2(1+\gamma)(2 k R)^{2 \gamma-2} e^{\pi \eta} \frac{|\Gamma(\gamma+i \eta)|^{2}}{|\Gamma(2 \gamma+1)|^{2}}
$$

For positrons, $Z$ has to be replaced by $-Z$ in the total expression (B6). If we are interested in the density matrix for electrons in negative energy states, we have to replace the $Z$ in (B6) by $-Z$ and the term $-\beta \gamma / W$ by its negative.

The first-forbidden $\beta$ decay in the $\xi$ approximation gives the same answers as the allowed $\beta$ decay, except for the fact that the allowed matrix elements have to be replaced by a certain linear combination of the firstforbidden ones (in case one does not observe the direction of the neutrino).

In order to show this, we first derive the density matrix for the first-forbidden $\beta$ decay. As shown in Sec. 1 , in the first-forbidden $\beta$ decay we have two types of matrix elements: Matrix elements which are of order $v / c$ in the nucleon velocities and occur by replacing the Fermi operator 1 by $\boldsymbol{\alpha}$ and the Gamow-Teller operator o by $\gamma_{5}$, and matrix elements which occur by replacing the Fermi operator 1 by $\mathbf{r}$ and the Gamow-Teller operator $\boldsymbol{d}$ by either one of the three operators $(\boldsymbol{\sigma} \cdot \mathbf{r})$, $[\boldsymbol{\sigma} \times \mathbf{r}]$, and the symmetric tensor with trace zero, $\sigma_{i} x_{j}+\sigma_{j} x_{i}-\frac{2}{3}(\boldsymbol{\sigma} \cdot \mathbf{r}) \delta_{i j}$. In the first case, electron and neutrino carry away no angular momentum, whereas in the second case the operator $\mathbf{r}$ origins from the fact that either electron or neutrino carry away one unit of angular momentum.

The $\xi$ approximation in first-forbidden $\beta$ decay is, physically speaking, characterized by the assumption that the Coulomb potential, which influences the electron, is large compared to its and the neutrino's kinetic energy. As discussed in Sec. 3, the formulation of this assumption is $\xi=Z \alpha / 2 R \gg W_{0}$, where $R$ is the nuclear radius, and $W_{0}$ is the maximum $\beta$-energy. (More accurately one should say that the underlying assumption of the $\xi$ approximation is that the product of $\xi$ with its particular linear combination of nuclear matrix elements is large compared to the product of the $k$ or $q$ with their particular linear combination of nuclear matrix elements. This condition is not always fulfilled even though the mathematical condition stated above may very well be satisfied.)

This condition implies that the neutrino carries away no angular momentum; therefore, we have to replace its plane wave function by one, exactly as in the allowed case. Inspection of Eq. (A15) shows, on the other hand, that terms with $\chi= \pm 1$ are of the same order in powers of $(k R)$, and are the only terms to be kept in the $\xi$ approximation. The corresponding wave functions are listed in Eqs. (B4) and (B5).

Contrary to the case of allowed transitions, however, we now have to take both parts of the wave functionthe parity conserving and the parity nonconserving part. ${ }^{8}$ The density matrix therefore consists of three parts: if we decompose $\psi^{(1) \mu}=\psi_{\mathrm{c}}^{( \pm) \mu}+\psi_{\text {n.c. }}{ }^{( \pm) \mu}$ into its parity-conserving and nonconserving parts, we obtain from Eq. (B1)

$$
\rho^{( \pm)}=\rho_{\text {c.e. }}{ }^{( \pm)}+\rho_{\text {ne.ne. }}{ }^{( \pm)}+\rho_{\text {c.nc. }}{ }^{( \pm)}+\rho_{\text {nc.. } .}{ }^{( \pm)} .
$$

The part denoted by $\rho_{\text {c.c. }}{ }^{ \pm}$was already given in Eq. (B6); a similar calculation shows that

$$
\left.\begin{array}{r}
\rho_{\text {nc. nc. }}{ }^{( \pm)}=\left(\boldsymbol{\alpha} \cdot \mathbf{r}_{0}\right)[(1-\gamma) /(1+\gamma)] \rho_{\text {c.c. }}{ }^{( \pm)}\left(\boldsymbol{\alpha} \cdot \mathbf{r}_{0}{ }^{\prime}\right), \\
\rho_{\text {c.ne. }}{ }^{( \pm)}=\left[\rho_{\text {ne. } .}{ }^{( \pm)}\right]^{*}=(+)[\alpha Z /(1+\gamma)] \rho_{\text {c.c. }}{ }^{( \pm)} \\
\cdot i\left(\boldsymbol{\alpha} \cdot \mathbf{r}_{0}{ }^{\prime}\right) .
\end{array}\right\}
$$

From Eq. (B8) we see that $\rho_{\text {nc.nc. }}$ differs from $\rho_{\text {c.c. }}$ by a constant factor, $(1-\gamma) /(1+\gamma)$, and by the fact that it is multiplied on both sides with $+\left(\boldsymbol{\alpha} \cdot \mathbf{r}_{0}\right)$, whereas $\rho_{\text {n..c. }}$ and $\rho_{\text {c.ne. }}$ differ from $\rho_{\text {c.c. }}$ by another constant factor and an either right-hand or left-hand side factor $i\left(\boldsymbol{\alpha} \cdot \mathbf{r}_{0}\right)$.

If we define the four vector

$$
A_{\mu}{ }^{m_{p} m_{n}}=C_{V}\left(\psi_{p}{ }^{* m_{p}} \gamma_{4} \gamma_{\mu} \psi_{n}{ }^{m_{n}}\right)-C_{A}\left(\psi_{p}{ }^{* m_{p}} \gamma_{4} \gamma_{\mu} \gamma_{5} \psi_{n}{ }^{m_{n}}\right),
$$

then the density matrix for the $\beta$ decay interaction is given by (see Appendix III) :

$$
\left.\begin{array}{rl}
\rho^{m_{p} m_{p}} m_{n} m_{n} \mu_{e} \mu_{e} \mu_{\mu} \mu_{\nu} \mu_{\nu^{\prime}}= & \sum_{\mu, \rho} A_{\mu}{ }^{m_{p}, m_{n}} A_{\rho}{ }^{* m_{p^{\prime}}, m_{n}{ }^{\prime}} \rho_{\nu} \\
& \cdot\left(1+\gamma_{5}\right) \gamma_{\mu} \gamma_{4} \rho_{e} \gamma_{4} \gamma_{\rho}\left(1+\gamma_{5}\right) .
\end{array}\right\}
$$

Here $\rho_{e}$ and $\rho_{\nu}$ are electron and neutrino density matrices, respectively. Our task is to show that the form of Eq. (B9) remains unchanged if we multiply $\rho_{e}$ on either side by $\left(\boldsymbol{\alpha} \cdot \mathbf{r}_{0}\right)$.

Now we have

$$
\begin{aligned}
-\sum_{\mu} i\left(\boldsymbol{\alpha} \cdot \mathbf{r}_{0}\right) \gamma_{4} \gamma_{\mu}\left(1+\gamma_{5}\right) A_{\mu}=+\left(\boldsymbol{\sigma} \cdot \mathbf{r}_{0}\right)\left(1+\gamma_{5}\right)(\boldsymbol{\sigma} \cdot \mathbf{A}) \\
-i\left(\boldsymbol{\sigma} \cdot \mathbf{r}_{0}\right)\left(1+\gamma_{5}\right) A_{4}=-i\left(\boldsymbol{\sigma} \cdot \mathbf{r}_{0}\right) A_{4}\left(1+\gamma_{5}\right) \\
+i\left[\left(\mathbf{r}_{0} \times \mathbf{A}\right) \cdot \boldsymbol{\sigma}\right]\left(\mathbf{1}+\gamma_{5}\right)+\left(\mathbf{r}_{0} \cdot \mathbf{A}\right)\left(1+\gamma_{5}\right) .
\end{aligned}
$$

If we redefine

we have

$$
\left.\begin{array}{l}
\mathbf{A}_{\imath}{ }^{\prime}=-\left[\mathbf{r}_{0} A_{4}-\left(\mathbf{r}_{0} \times \mathbf{A}\right)\right] \\
A_{4}{ }^{\prime}=\left(\mathbf{r}_{0} \cdot \mathbf{A}\right),
\end{array}\right\}
$$

$$
-\sum_{\mu} i\left(\boldsymbol{\alpha} \cdot \mathbf{r}_{0}\right) \gamma_{4} \gamma_{\mu}\left(1+\gamma_{5}\right) A_{\mu}=\sum_{\mu} \gamma_{4} \gamma_{\mu}\left(1+\gamma_{5}\right) A_{\mu}{ }^{\prime} .
$$

That means, that by the simple replacement $A_{\mu} \rightarrow A_{\mu}{ }^{\prime}$, we obtain the same form of the density matrix, or: by changing the definition of nuclear matrix elements, we can switch from the formulas for the allowed to the formulas for the first-forbidden $\beta$ decay in the $\xi$ approxi-

\footnotetext{
${ }^{8}$ More precisely, both the part of zeroth order in $\mathbf{r}_{0}$ taken in the allowed case and the part of first order in $\mathbf{r}_{0}$ neglected before will come into play, the first one for the matrix elements $\int \gamma_{5}$ and $\int \boldsymbol{\alpha}$, the other one for the other matrix elements mentioned above.
} 
mation. The same is true for either left- or right-hand multiplication of $\rho_{o}$ with either $\left(\boldsymbol{\alpha} \cdot \mathbf{r}_{0}\right)$ or $\left(\boldsymbol{\alpha} \cdot \mathbf{r}_{0}{ }^{\prime}\right)$, and therefore our statement made above has been shown to be true.

The change in the matrix elements which has to be made can also easily be deduced from the above formulas: Since $(1-\gamma) /(1+\gamma) \approx(Z \alpha)^{2} / 4$ and $Z \alpha /(1+\gamma)$ $\approx Z \alpha / 2$, our density matrix is unchanged for the parity conserving part of the electron wave function, and multiplied by $-i \xi(\boldsymbol{\alpha} \cdot \mathbf{r})$ or its hermetian conjugate for the parity nonconserving part. For the nuclear matrix elements, this leads immediately to a prescription for the replacement of matrix elements.

Table $\mathrm{V}$ indicates which allowed matrix elements have to be replaced by which forbidden ones if one wants to write down formulas for forbidden decay.

From Table $\mathrm{V}$ it becomes apparent that all terms which are not of order $v / c$ in the nucleon velocity are multiplied by $\xi=Z \alpha / 2 R$, where $R$ is the nuclear radius (see Appendix VI). This explains the term $\xi$ approximation and, at the same time, shows that this word is not a particularly characteristic one because of the occurrence of terms of order $v / c$ which are independent of $\xi$.

\section{APPENDIX III. DERIVATION OF DENSITY MATRIX FOR $\beta$-DECAY INTERACTION}

We derive here the formulas of $\beta^{-}$emission. The cases of the orbital electron capture and $\beta^{+}$emission are considered in Appendix VII. We define the $\gamma$ matrices in the usual way:

$\gamma_{i}=-i \beta \alpha_{i} ; \gamma_{4}=-\beta ; \sigma_{i}=+\gamma_{5} \alpha_{i}$

$$
\gamma_{5}=+\gamma_{1} \gamma_{2} \gamma_{3} \gamma_{4} ; i=1,2,3 \text {. }
$$

The interaction Hamiltonian then has the form (we sum automatically over indices that occur twice, and suppress the summation over the nucleons, which has to be carried along implicitly throughout all our expressions) :

$$
\begin{aligned}
& H_{\beta}=C_{V}\left(\psi_{p}{ }^{*} \gamma_{4} \gamma_{\mu} \tau-\psi_{n}\right)\left[\psi_{e}^{*} \gamma_{4} \gamma_{\mu}\left(1+\gamma_{5}\right) \psi_{\nu}\right] \\
& -C_{A}\left(\psi_{p}^{*} \gamma_{4} \gamma_{\mu} \tau^{-} \gamma_{5} \psi_{n}\right)\left[\psi_{e}^{*} \gamma_{4} \gamma_{\mu}\left(1+\gamma_{5}\right) \psi_{\nu}\right]+\text { herm. conj. } \\
& =-C_{V}\left(\psi_{p}^{*} \boldsymbol{\alpha} \tau-\psi_{n}\right)\left[\psi_{e}^{*} \boldsymbol{\alpha}\left(1+\gamma_{5}\right) \psi_{\nu}\right] \\
& +C_{V}\left(\psi_{p}^{*} \tau^{-} \psi_{n}\right)\left[\psi_{e}^{*}\left(1+\gamma_{5}\right) \psi_{\nu}\right] \\
& +C_{A}\left(\psi_{p}^{*} \boldsymbol{\partial} \tau \psi_{n}\right)\left[\psi_{e}^{*} \boldsymbol{\partial}\left(1+\gamma_{5}\right) \psi_{\nu}\right] \\
& -C_{A}\left(\psi_{p}^{*} \gamma_{5} \tau^{-} \psi_{n}\right)\left[\psi_{e}^{*}\left(1+\gamma_{5}\right) \psi_{\nu}\right]+\text { herm. conj. } \\
& =-\left[\psi_{p}{ }^{*}\left(C_{V}-C_{A} \gamma_{5}\right) \boldsymbol{\alpha} \tau^{-} \psi_{n}\right]\left[\psi_{e}{ }^{*} \boldsymbol{\alpha}\left(1+\gamma_{5}\right) \psi_{\nu}\right] \\
& +\left[\psi_{p}{ }^{*}\left(C_{V}-C_{A} \gamma_{5}\right) \tau^{-} \psi_{n}\right]\left[\psi_{e}^{*}\left(1+\gamma_{5}\right) \psi_{\nu}\right]+\text { herm. conj. }
\end{aligned}
$$

Here, $\psi_{p}$ and $\psi_{n}$ represent the initial and final states of the nucleus in a $\beta^{-}$-decay, and $\psi_{e}$ and $\psi_{\nu}$ are the negaton and neutrino wave functions. $\tau^{-}$is the component of the isotopic spin operator which changes a neutron into a proton. The integration is over lepton and nucleon space coordinates simultaneously; therefore, we write explicitly

$$
\begin{aligned}
H_{\beta}=-\int d \tau_{N} d \tau_{L}\left[\psi_{p}^{*}\left(C_{V}-C_{A} \gamma_{5}\right) \boldsymbol{\alpha} \tau \psi_{n}\right]_{\mathbf{r}_{N}} \\
\left(\psi_{e}^{*} \boldsymbol{\alpha}\left(1+\gamma_{5}\right) \psi_{\nu}\right]_{\mathbf{r}_{L}} \delta\left(\mathbf{r}_{N}-\mathbf{r}_{L}\right)+\int d \tau_{N} d \tau_{L} \\
{\left[\psi_{p}^{*}\left(C_{V}-C_{A} \gamma_{5}\right) \tau \psi_{n}\right]_{\mathbf{r}_{N}}\left(\psi_{e}^{*}\left(1+\gamma_{5}\right) \psi_{\nu}\right] \mathbf{r}_{L} \delta\left(\mathbf{r}_{N}-\mathbf{r}_{L}\right) . }
\end{aligned}
$$

Now, we use the formal identity

$$
\begin{aligned}
\delta\left(\mathbf{r}_{1}-\mathbf{r}_{2}\right)=\left(r_{1} r_{2}\right)^{-1} \delta\left(r_{1}-r_{2}\right) & \sum_{L, M}(-)^{L+M} i^{L} \\
& Y_{L}^{M}\left(\mathbf{r}_{0}^{1}\right) i^{L} Y_{L^{M}}\left(\mathbf{r}_{0}^{2}\right)
\end{aligned}
$$

where $\mathbf{r}_{0}=\mathbf{r} / r$, and introduce the definition:

$$
\begin{aligned}
& T_{K L 1}{ }^{M}=\sum_{v} c(1, L, K ; M-v, v) \gamma_{5} \sigma^{M-v} i^{L} Y_{L^{v}} \\
& T_{K L 0}{ }^{M}=\sum_{v} c(0, L, K ; M-v, v) i^{L} Y_{L^{v}}
\end{aligned}
$$

or, in compact form,

$T_{K L \gamma}{ }^{M}=\sum_{v} c(\gamma, L, K ; M-v, v)\left[\gamma_{5} \sigma^{M-v}\right]^{\gamma} i^{L} Y_{L} v$,

where $\gamma=0,1$. The operators $T_{K L \gamma}{ }^{M}$ are irreducible tensor operators of rank $K$, and help us to carry through our main objective: The multipole expansion of the $\beta$ interaction and the classification of the degree of forbiddenness of a transition with the help of this expansion. Introducing Eqs. (C2a) and (C2b) into Eq. (C2), we obtain ${ }^{9}$

$$
\begin{aligned}
& H_{\beta}=\sum_{K L \gamma \mu}(-)^{K+\mu} \int_{0}^{\infty} r^{2} d r \\
& \begin{aligned}
\left\{\int d \Omega_{N}\left[\psi_{p}{ }^{*}\left(C_{V}-C_{A} \gamma_{5}\right) T_{K L}{ }^{\mu} \tau^{-} \psi_{n}\right]\right\}_{r_{N}=r} \\
\cdot\left\{\int d \Omega_{L}\left[\psi_{e}{ }^{*}\left(1+\gamma_{5}\right) T_{K L \gamma}{ }^{-\mu} \psi_{\nu}\right]\right\}_{r_{L}=r}
\end{aligned}
\end{aligned}
$$

Here, $\gamma$ takes the values zero and one, $K$ and $L$ take all integer non-negative values, and $\mu$ is integer and restricted by $-K \leq \mu \leq K$.

Now we insert the Coulomb-field solution for $\psi_{e}$, obtained in Appendix I, and the corresponding planewave solution for $\psi_{\nu}$, and apply the Wigner-Eckart theorem. According to Appendix I we have

$$
\begin{aligned}
\psi_{e}^{( \pm) m_{e}} & =\sum_{\chi \neq 0}\{4 \pi[2 l(\chi)+1]\}^{\frac{1}{2}} c\left[l(\chi) \frac{1}{2} j(\chi) ; 0 m_{e}\right] e^{ \pm i \Delta_{x}} \psi_{\chi}^{m_{e}}, \\
\psi_{\nu}^{m_{\nu}} & =\sum_{\chi \neq 0}\{4 \pi[2 l(\chi)+1]\}^{\frac{1}{2}} c\left[l(\chi) \frac{1}{2} j(\chi) ; 0 m_{\nu}\right] \psi_{\chi}^{m_{\nu}} .
\end{aligned}
$$

Here, $\psi_{x}{ }^{m_{e}}$ was defined in Eq. (A11), whereas $\psi_{x}{ }^{m_{\nu}}$ is the corresponding solution obtained by $Z \rightarrow 0$ :

$\psi_{\chi}{ }^{m_{\nu}}=\sqrt{2}^{-1}\left[j_{l(\chi)}(q r)+\operatorname{Sg} \chi_{\nu} i\left(\boldsymbol{\alpha} \cdot \mathbf{r}_{0}\right) j_{l(-\chi)}(q r)\right] \phi_{\chi}{ }^{m_{\nu}}$. (C4a)

${ }^{9}$ From now on we restrict the discussion to $\beta^{-}$emission. The case of $\beta^{+}$emission or $\beta^{-}$capture is treated in Appendix VII. 
$k$ and $q$ are negaton and neutrino momentum, respectively, and we use the units $\hbar=m=c=1 . j_{l}$ are spherical Bessel functions. Equations (C4) assume that the direction of quantization is chosen along the direction of propagation of the particles; if we refer instead to one common coordinate system, the first equation of (C4) reads, for example,

$$
\begin{aligned}
& \psi_{e}^{( \pm) m_{e}}=\sum_{\chi \neq 0} \sum_{v} D_{j(\chi)}{ }^{v m_{e}}\{\{4 \pi[2 l(\chi)+1]\}^{\frac{1}{2}} \\
& \cdot c\left[l(\chi) \frac{1}{2} j(\chi) ; 0 m_{e}\right] \exp \left( \pm i \Delta_{\chi}\right) \psi_{\chi}{ }^{v} .
\end{aligned}
$$

Here, $D_{j(\chi)^{v m}}(\theta)$ is the rotation matrix in the conventional definition; it depends on the magnetic quantum numbers $v$ and $m_{e}$ in the two coordinate systems, and on the Euler angles connecting these two systems, in the way defined by Rose (see Appendix VIII). It fulfills the orthogonality relation

$$
\int d \Omega D_{j}{ }^{v m} D_{j^{\prime}}{ }^{* v^{\prime} m^{\prime}}=[4 \pi /(2 j+1)] \delta_{j j^{\prime}} \delta_{v v^{\prime}} \delta_{m m^{\prime}}
$$

For the evaluation of $H_{\beta}$, we realize that it depends on the four magnetic quantum numbers $m_{e}$ for the electron, $m_{\nu}$ for the neutrino, $m_{n}$ and $m_{p}$ for the initial and final nuclear states. If we define the reduced matrix elements by the relation ${ }^{10}$

$$
\begin{aligned}
\left(j m\left|\begin{array}{ll}
O & \\
& \Lambda
\end{array}\right| j^{\prime} m^{\prime}\right) & \\
& =(-)^{j-m}\left(\begin{array}{ccc}
j & \Lambda & j^{\prime} \\
-m & \mu & m^{\prime}
\end{array}\right) j\left\|0_{\Lambda}\right\| j^{\prime}
\end{aligned}
$$

where

$$
\left(\begin{array}{ccc}
j & \Lambda & j^{\prime} \\
& & \\
-m & \mu & m^{\prime}
\end{array}\right)
$$

are Wigner $3-j$ coefficients (see Appendix VIII), we obtain for $H_{\beta}^{11}$ :

$$
\begin{aligned}
& H_{\beta}=\sum_{K L \gamma \mu}(-)^{K+\mu} \sum_{\chi_{\varepsilon}, \chi_{\nu} \neq 0} \sum_{v_{e} v_{\nu}} D_{j\left(\chi_{e}\right)} *_{e} m_{e} m_{e}\left(\theta_{e}\right) D_{j\left(\chi_{\nu}\right)}{ }^{v_{\nu} m_{\nu}}\left(\theta_{\nu}\right) \\
& \cdot 4 \pi \cdot\left\{\left[2 l\left(\chi_{e}\right)+1\right]\left[2 l\left(\chi_{\nu}\right)+1\right]\right\}^{\frac{1}{2}} \\
& \cdot c\left[l\left(\chi_{e}\right) \frac{1}{2} j\left(\chi_{e}\right) ; 0 m_{e}\right] c\left[l\left(\chi_{\nu}\right) \frac{1}{2} j\left(\chi_{\nu}\right) ; 0 m_{\nu}\right]
\end{aligned}
$$

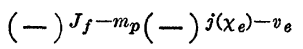

$$
\begin{aligned}
& \times\left(\begin{array}{ccc}
J_{f} & K & J i \\
-m_{p} & \mu & m_{J}
\end{array}\right)\left(\begin{array}{ccc}
j\left(\chi_{e}\right) & K j & \left(\chi_{\nu}\right) \\
-v_{e} & -\mu & v_{\nu}
\end{array}\right) \exp \left(i \Delta_{\chi_{e}}\right) \\
& \cdot \int_{0}^{\infty} r^{2} d r\left\langle J_{f}\left\|\left(C_{\nu}-C_{A} \gamma_{5}\right) T_{K L \gamma} \tau^{-}\right\| J i\right\rangle_{r_{N}=r} \\
& \left\langle j\left(\chi_{e}\right)\left\|\left(1+\gamma_{5}\right) T_{K L_{\gamma}}\right\| j\left(\chi_{\nu}\right)\right\rangle_{r_{L}=r} .
\end{aligned}
$$

${ }^{10}$ With this definition, the reduced matrix elements will be real (see reference ALD 57). They are not, however, symmetric. Instead, since

we have

$$
T_{K L \gamma}{ }^{* M}=(-)^{K-\gamma-M} T_{K L \gamma^{-M}},
$$

$$
\left.\left\langle j_{1}\left\|T_{K L \gamma}\right\| j\right\rangle^{*}=(-)\right)^{K-\gamma+j-j_{1}}\left\langle j\left\|T_{K L \gamma}\right\| j_{1}\right\rangle
$$

${ }^{11}$ Here, and from now on, we use only incoming scattered waves for the solution of the electron wave function, as corresponds to the physical condition.
This way, $H_{\beta}$ can be written as a sum of products. Some terms in the product contain all the dependence on the magnetic quantum numbers and angles, and therefore all the information necessary to calculate the form of the various functions one can measure (spectrum, angular correlation functions, etc.). The residual information which contains all the dynamics of the process is contained in the residual matrix elements, the evaluation of which is considered in the next appendix.

By multiplying $H_{\beta}$ with its complex conjugate, we obtain the density matrix for $\beta$ decay. Since in the experiments on first-forbidden decay the direction of the neutrino usually is not observed, we integrate this product over the neutrino angles and sum over the neutrino magnetic quantum numbers.

We define

$$
\begin{array}{r}
C_{K L \gamma}\left[J_{i}, J_{f} ; j\left(\chi_{e}\right), j\left(\chi_{\nu}\right)\right] \\
=\int_{0}^{\infty} r^{2} d r\left\langle J_{f}\left\|\left(C_{V}-C_{A} \gamma_{5}\right) T_{K L \gamma} \tau^{-}\right\| J i\right\rangle \\
\left\langle j\left(\chi_{e}\right)\left\|\left(1+\gamma_{5}\right) T_{K L \gamma}\right\| j\left(\chi_{\nu}\right)\right\rangle
\end{array}
$$

and obtain

$$
\begin{aligned}
& \rho\left(m_{p} m_{p}^{\prime} ; m_{n} m_{n}^{\prime} ; m_{e} m_{e}^{\prime}\right) \\
& =(4 \pi)^{3} \sum_{K K^{\prime} L L^{\prime} \gamma \gamma^{\prime} \chi_{e} \chi_{e} \chi_{\nu} J}(-)^{J+j\left(\chi_{\nu}\right)-j\left(\chi_{e}\right)-j\left(\chi_{e}\right)} \\
& \cdot(-)^{+m_{p}-m_{n}-m_{e}}\left\{\left[2 l\left(\chi_{e}\right)+1\right]\left[2 l\left(\chi_{e}^{\prime}\right)+1\right]\right\}^{\frac{1}{2}} \\
& \cdot c\left[l\left(\chi_{e}\right) \frac{1}{2} j\left(\chi_{e}\right) ; 0 m_{e}\right] c\left[l\left(\chi_{e}^{\prime}\right) \frac{1}{2} j\left(\chi_{e}^{\prime}\right) ; 0 m_{e}^{\prime}\right] \\
& \cdot c\left[j\left(\chi_{e}\right) j\left(\chi_{e}^{\prime}\right) J ;-m_{e}, m_{e}^{\prime}\right]\left(2 J_{i}+1\right)^{-1} \\
& \cdot c\left(J_{f} K J_{i} ;-m_{p}, m_{p}-m_{n}\right) c\left(J_{f} K^{\prime} J_{i} ;-m_{p}{ }^{\prime}, m_{p}{ }^{\prime}-m_{n}{ }^{\prime}\right) \\
& \cdot c\left(K K^{\prime} J ; m_{p}-m_{n},-m_{p}{ }^{\prime}+m_{n}{ }^{\prime}\right) \\
& \text { - } W\left[j\left(\chi_{e}\right) K j\left(\chi_{e}^{\prime}\right) K^{\prime} ; j\left(\chi_{\nu}\right) J\right] D_{J}^{m_{p}-m_{p}{ }^{\prime}-m_{n}+m_{n}, m_{e} e^{\prime}-m_{e}} \\
& \text { - } C_{K L \gamma}\left[J_{i} J_{f} ; j\left(\chi_{e}\right) j\left(\chi_{\nu}\right)\right] C_{K^{\prime} L^{\prime} \gamma^{\prime}}{ }^{*} \\
& \cdot\left[J_{i} J_{f} ; j\left(\chi_{e}^{\prime}\right) j\left(\chi_{\nu}\right)\right] \times \exp \left[+i\left(\Delta_{\chi_{e}}-\Delta_{\chi^{\prime}}\right)\right]
\end{aligned}
$$

Application of some Racah algebra yields the final form of the density matrix from which it is easily possible to calculate all the measurable quantities which do not imply a measurement of the neutrino directions: In Eq. (C8) we have a product of three Clebsch-Gordan coefficients involving only $l\left(\chi_{e}\right), l\left(\chi_{e}^{\prime}\right)$, $j\left(\chi_{e}\right), j\left(\chi_{e}^{\prime}\right)$ and $J$, and another product of three such coefficients involving only $K, K^{\prime}, J_{i}, J_{f}$, and $J$. Both these products can be written separately as a sum over a product involving a $9-j$ coefficient and in this way we obtain the following final formula for $\rho$ : We define 
the particle parameters $B_{J}^{u, t}\left(K, K^{\prime}\right)$ by $\left[j_{c} \equiv j\left(\chi_{c}\right)\right.$ etc. $]$

$$
\begin{aligned}
& B_{J^{u, l}}\left(K, K^{\prime} ; J_{f}, J_{i}\right)=4 \pi^{2} \frac{2 u+1}{2 J_{i}+1} \frac{[2(2 t+1)]^{\underline{1}}}{\left[(2 K+1)\left(2 K^{\prime}+1\right)(2 J+1)\right]^{1}}\left(\begin{array}{ccc}
K & K^{\prime} & J \\
1 & -1 & 0
\end{array}\right)^{-1} \sum_{L L^{\prime} \gamma \gamma^{\prime} x_{e} \chi_{e}^{\prime} \chi_{\nu}}(-)^{j_{v}+j_{e}+l_{e}^{\prime}-1} \\
& \cdot\left\{\left[2 l\left(\chi_{e}\right)+1\right]\left[2 l\left(\chi_{e}^{\prime}\right)+1\right]\left[2 j\left(\chi_{e}\right)+1\right]\left[2 j\left(\chi_{e}^{\prime}\right)+1\right]\right\}^{2}\left(\begin{array}{ccc}
l\left(\chi_{e}\right) & l\left(\chi_{e}^{\prime}\right) & u \\
0 & 0 & 0
\end{array}\right)\left\{\begin{array}{ccc}
K & K^{\prime} & J \\
j\left(\chi_{e}^{\prime}\right) & j\left(\chi_{e}\right) & j\left(\chi_{\nu}\right)
\end{array}\right\}\left\{\begin{array}{ccc}
l\left(\chi_{e}\right) & l\left(\chi_{e}^{\prime}\right) & u \\
\frac{1}{2} & \frac{1}{2} & t \\
j\left(\chi_{e}\right) & j\left(\chi_{e}^{\prime}\right) & J
\end{array}\right\} \\
& \cdot \exp \left[+i\left(\Delta_{\chi_{e}}-\Delta_{\chi^{\prime}{ }_{e}}\right) C_{K L \gamma}\left[J_{i} J_{f} ; j\left(\chi_{e}\right) j\left(\chi_{v}\right)\right] C_{K^{\prime} L^{\prime} \gamma^{\prime}} *\left[J_{i} J_{f} ; j\left(\chi_{\theta}^{\prime}\right) j\left(\chi_{\nu}\right)\right]\right.
\end{aligned}
$$

and the quantities $F_{J^{u, t}}\left(K, K^{\prime} ; J_{f}, J_{i}\right)$ by

$F_{J^{u, t}}\left(K, K^{\prime} ; J_{f}, J_{i}\right)=\left[\left(2 J_{i}+1\right)\left(2 J_{f}+1\right)(2 J+1)(2 K+1)\left(2 K^{\prime}+1\right)(2 u+1)(2 t+1)\right]^{\frac{1}{2}}$

and obtain

$$
\cdot(-)^{-K-J-1}\left(\begin{array}{ccc}
K & K^{\prime} & J \\
1 & -1 & 0
\end{array}\right)\left\{\begin{array}{lll}
J_{i} & J_{f} & K \\
t & u & J \\
J_{i} & J_{f} & K^{\prime}
\end{array}\right\}
$$

$$
\begin{aligned}
& \rho\left(m_{p} m_{p}{ }^{\prime} ; m_{n} m_{n}{ }^{\prime} ; m_{e} m_{e}{ }^{\prime}\right)=16 \pi \sum_{u u^{\prime} t t^{\prime} K K^{\prime} J}\left[\left(2 J_{i}+1\right) / 2\left(2 J_{f}+1\right)\right]^{\frac{1}{2}}(-)^{K+K^{\prime}+J_{i}+\frac{1}{2}+m_{p^{\prime}}-m_{n}-m_{e}+t+u^{\prime}-J-J_{f}} \\
& \cdot c\left(t, u, J ; m_{n}-m_{n}{ }^{\prime}, m_{p}{ }^{\prime}-m_{p}\right) c\left(J_{f} J_{f} u ; m_{p},-m_{p}{ }^{\prime}\right) c\left(J_{i} J_{i} t ; m_{n},-m_{n}{ }^{\prime}\right) c\left(t^{\prime} u^{\prime} J ; m_{e}{ }^{\prime}-m_{e}, 0\right) c\left(\frac{1}{2} \frac{1}{2} t^{\prime} ; m_{e},-m_{e}{ }^{\prime}\right)
\end{aligned}
$$

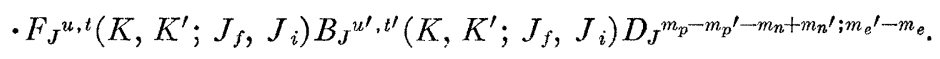

Equation (C9c) shows that except for geometrical factors the density matrix is completely characterized by the particle parameters $B_{J}$. The interpretation of the $B_{J}$ 's is fairly simple: The interaction was expanded into multipoles. Each term of the expansion is characterized by an irreducible tensor $T_{K L \gamma}$ of rank $K$. Each $B_{J}$ contains a sum over all possible products of the reduced nuclear matrix elements of two tensor operators $T_{K L \gamma}$ and $T_{K^{\prime} L^{\prime} \gamma^{\prime}}$ with $K$ and $K^{\prime}$ fixed (they are numbers that characterize $B_{J}$ ). The possible values of $K$ and $K^{\prime}$ are given by the selection rule

$$
\left|J_{f}-J_{i}\right| \leq K, K^{\prime} \leq J_{f}+J_{i}
$$

The operators $T_{K L \gamma}$ and $T_{K^{\prime} L^{\prime} \gamma^{\prime}}$ occur not only in the nuclear matrix elements, but also in the reduced lepton matrix elements, where they stand between a wave function for a neutrino with spin $j\left(\chi_{v}\right)$ and a wave function for an electron with spin $j\left(\chi_{e}\right)$ and $j\left(\chi_{e}^{\prime}\right)$, respectively. Of course, for given $K$ and $K^{\prime}$ the numbers $j\left(\chi_{e}\right), j\left(\chi_{e}^{\prime}\right)$, and $j\left(\chi_{v}\right)$ are limited by

$$
\begin{gathered}
\left|j\left(\chi_{e}\right)-j\left(\chi_{v}\right)\right| \leq K \leq j\left(\chi_{e}\right)+j\left(\chi_{v}\right), \\
\left|j\left(\chi_{e}^{\prime}\right)-j\left(\chi_{v}\right)\right| \leq K^{\prime} \leq j\left(\chi_{e}^{\prime}\right)+j\left(\chi_{v}\right) .
\end{gathered}
$$

Besides $K$ and $K^{\prime}$, also $J$ is a number that characterizes $B_{J}$-it gives the degree of $J$ the Legendre polynominal in the angular distribution of the electron (or its polarization) in the final expression. Naturally,

$$
\left|j\left(\chi_{e}\right)-j\left(\chi_{e}^{\prime}\right)\right| \leq J \leq j\left(\chi_{e}\right)+j\left(\chi_{e}^{\prime}\right) \text {. }
$$

On the other hand, $J$ also fulfills the selection rule

$$
\left|K-K^{\prime}\right| \leq J \leq K+K^{\prime}
$$

For fixed numbers, $J, K, K^{\prime}$, the choice of which is restricted by Eqs. (C10) and (C13), each $B$ contains a sum over all lepton matrix elements with the spins of the leptons restricted by Eqs. (C11) and (C12). Clearly, this sum is infinite.

The meaning of the auxiliary indices $u$ and $t$ becomes clear when one looks at Eq. (C9c): If we do not measure the spin of the electron, $t=0$ and $u=J$. In this case, the definition of $B_{J}^{J, 0}\left(K, K^{\prime}\right)$ is such that it reduces to the particle parameter $b_{J}\left(K, K^{\prime}\right)$ defined by Alder, Stech, and Winther (ALD57):

$$
B_{J}{ }^{, 0}\left(K, K^{\prime} ; J_{f}, J_{i}\right) \equiv b_{J}\left(K, K^{\prime}\right)
$$

If we measure the spin of the electron, $t=1$, and $B_{J}$ is a straightforward generalization of $b_{J}\left(K, K^{\prime}\right)$. In that case, $u$ can have the values $J-1, J$, and $J+1$.

The density matrix depends also on the geometrical quantities $F_{J}{ }^{u, t}\left(K, K^{\prime} ; J_{j}, J_{i}\right)$. Here, $t=0$ for unaligned nuclei, and $u=0$ if we do not consider the case of a radiation following the formation of the final nucleus. Otherwise, $u$ and $t$ depend on the degree of polarization and angular distribution of the radiation (i.e., its multipolarity), respectively. We have defined $F_{J}$, such that $F_{J}{ }^{0, J}\left(K, K^{\prime} ; J_{f}, J_{i}\right) \equiv F_{J}\left(K, K^{\prime}, J_{f}, J_{i}\right)$ as defined by Alder, Stech, and Winther (ALD57).

The calculation of various measurable quantities 
from Eq. (C9c) is now extremely simple and is carried through in the following appendix.

\section{APPENDIX IV. CALCULATIONS OF VARIOUS MEASURABLE OUANTITIES AS FUNCTIONS OF PARAMETERS $B_{J}$ AND $\boldsymbol{F}_{J}$}

In this appendix, we give formulas for spectrum shape, $\beta-\gamma$ correlation function, etc., in terms of the parameters $B_{J}$ and $F_{J}$. The experimental quantities of interest are: (a) the shape of the spectrum; (b) the electron polarization from unoriented nuclei; (c) the $\beta$ - $\gamma$ angular correlation function; (d) the $\beta$-circularly polarized $\gamma$ angular correlation function; (e) the polarized $\beta-\gamma$ angular correlation functions for various directions of the $\beta$ polarization; (f) the angular distribution of electrons from oriented nuclei; and $(\mathrm{g})$ the angular distribution of $\gamma$ 's, following the $\beta$ decay from oriented nuclei.

In order to calculate these quantities, we first define the alignment or polarization of the initial nucleus, and a density matrix for the $\gamma$ decay corresponding to the $\beta$-decay density matrix defined in the preceding appendix.

For a characterization of the alignment or polarization of the initial nucleus, we assume that the occupation numbers of the magnetic substates $a_{m_{n}}$ are known, if we choose the quantization axis along the alignment axis. (We have, of course, $\sum_{m} a_{m}=2 J_{i}+1$.)

The density matrix for a $\gamma$ radiation from a nuclear level with spin $J_{f}$ to another level with spin $J_{f f}$ is given by (ALD 57)

$$
\rho_{\gamma}\left(m_{p} m_{p}{ }^{\prime} \tau\right)=\sum_{k \lambda \lambda^{\prime}}(2 k+1)^{\frac{1}{2}} \tau^{k}(-)^{J_{f}+m_{p} F_{k}{ }^{0, k}}\left(\lambda, \lambda^{\prime}, J_{f f}, J_{f}\right)\left(2 J_{f}+1\right)^{-3}\left(\begin{array}{ccc}
J_{f} & J_{f} & k \\
-m_{p} & m_{p}{ }^{\prime} & m_{p}-m_{p}{ }^{\prime}
\end{array}\right) \delta_{\lambda} \delta_{\lambda^{\prime}} D_{k}{ }^{*} m_{p^{\prime}-m_{p^{\prime}, 0}\left(\theta_{\gamma}\right)}
$$

Here, $F_{k}{ }^{0, k}$ has been defined in Eq. (C9b), and $\delta_{\lambda}$ is the usual transition amplitude for $2^{\lambda}$ pole radiation. In Eq. (D1), the summation over the final nuclear magnetic substates has already been carried through. The symbol $\tau$ denotes the circular polarization of the $\gamma$ ray, it is +1 for right-handed and -1 for left-handed circularly polarized light.

Thus we obtain:

(a) For the spectrum shape factor (KON 41)

$$
\begin{aligned}
& 32 \pi^{2} C=\frac{1}{2 J_{i}+1_{m_{p} m_{p} m_{n} m_{n} m_{n}^{\prime} m_{e} m_{e^{\prime}}}} \delta_{m_{e} m_{e^{\prime}}} \delta_{m_{p} m_{p^{\prime}}} \delta_{m_{n} m_{n^{\prime}}} \int d \Omega_{e} \rho\left(m_{p} m_{p}{ }^{\prime} ; m_{n} m_{n}{ }^{\prime} ; m_{e} m_{e}{ }^{\prime}\right) \\
& =4^{3} \pi^{2} \sum_{K} F_{0}^{0,0}\left(K, K ; J_{f}, J_{i}\right) B_{0}^{0,0}\left(K, K ; J_{f}, J_{i}\right)
\end{aligned}
$$

or

$$
C=2 \sum_{K} B_{0}^{0,0}\left(K, K ; J_{f}, J_{i}\right)
$$

(b) For the electron polarization from unoriented nuclei

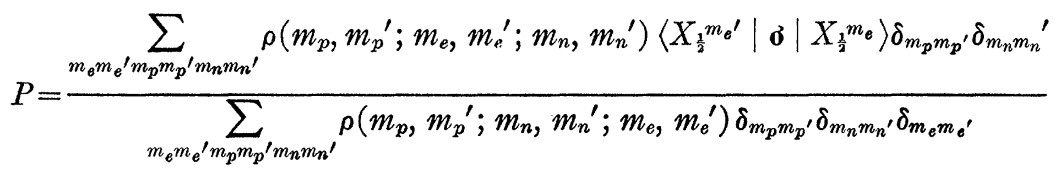

Since the summation over $m_{p}=m_{p}{ }^{\prime}$ and $m_{n}=m_{n}{ }^{\prime}$ gives $u=t=0$ and therefore $J=0$, which has the consequence $m_{e}=m_{e}^{\prime}$, the direction of the electron polarization is given by its direction of motion and has the value

$$
P=\frac{+1}{\sqrt{3}} \frac{\sum_{K} B_{0}^{1,1}\left(K, K ; J_{f} J_{i}\right)}{\sum_{K} B_{0}^{0,0}\left(K, K ; J_{f}, J_{i}\right)} .
$$

(c) For the $\beta-\gamma$ angular correlation function

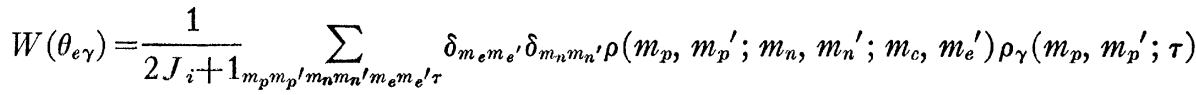

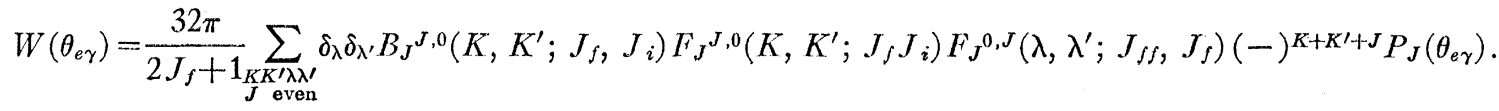


(d) For the $\beta$ circularly polarized $\gamma$-angular correlation function

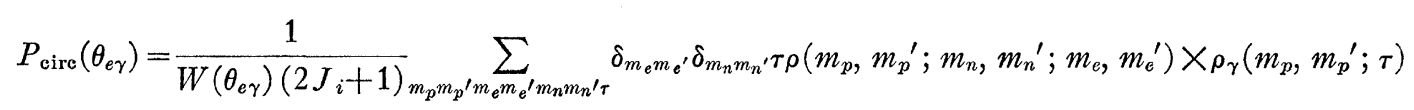

or

$$
\begin{aligned}
P_{\text {eirc }}\left(\theta_{e \gamma}\right)=\frac{1}{W\left(\theta_{e \gamma}\right)} \frac{32 \pi}{2 J_{f}+1} \sum_{\substack{K K^{\prime} \lambda \lambda^{\prime \prime} \\
\text { dd }}} \delta_{\lambda} \delta_{\lambda^{\prime}} B_{J}^{J, 0}\left(K, K^{\prime} ; J_{f}, J_{i}\right) F_{J^{J, 0}}\left(K, K^{\prime} ; J_{f}, J_{i}\right) \\
\cdot F_{J}^{0, J}\left(\lambda, \lambda^{\prime} ; J_{f f}, J_{f}\right)(-)^{K+K^{\prime}+J} P_{J}\left(\theta_{e \gamma}\right) .
\end{aligned}
$$

(e) For the angular correlation between the polarization of the $\beta$ 's and subsequently emitted $\gamma$ rays:

This correlation function has three components corresponding to the three possible directions of the polarization vector of the negatons. If we choose the direction of propagation of the electrons as quantization axis, we obtain

$$
P_{\mu}\left(\theta_{e \gamma}\right)=\frac{1}{W\left(\theta_{e \gamma}\right)} \cdot \sum_{m_{p} m_{p}^{\prime} m_{n} m_{n}^{\prime} m_{e} m_{e}^{\prime} \tau} \delta_{m_{n} m_{n}}\left\langle X_{\frac{1}{2}}^{m_{e}}{ }^{\prime}\left|\boldsymbol{\delta}_{\mu}\right| X_{\frac{1}{2}}^{m_{e}}\right\rangle \rho\left(m_{p}, m_{p}{ }^{\prime} ; m_{n}, m_{n}^{\prime} ; m_{e}, m_{e}{ }^{\prime}\right) \times \rho_{\gamma}\left(m_{p}, m_{p}{ }^{\prime} ; \tau\right)
$$

or

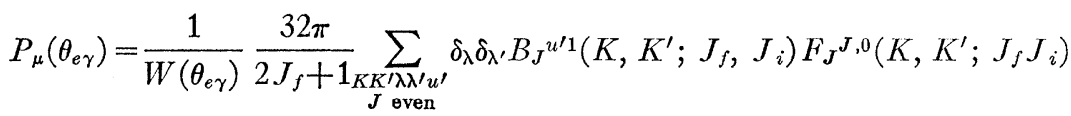

$$
\begin{aligned}
& \cdot F_{J}^{0 J}\left(\lambda, \lambda^{\prime} ; J_{f f}, J_{f}\right)(-)^{K+K^{\prime}+u^{\prime}} c\left(1 u^{\prime} J ; \mu, 0\right) D_{J}{ }^{*_{\mu, 0},}\left(\theta_{e \gamma}\right) .
\end{aligned}
$$

Here, $\mu=-1,0,1$. The longitudinal polarization is, therefore, given by $P_{0}$, the transverse polarization in the reaction plane by

$$
P_{t \| l}=i \sqrt{2}\left(P_{1}+P_{-1}\right)
$$

and the polarization perpendicular to the reaction plane by

$$
P_{t \perp}=\sqrt{2}\left(P_{-1}-P_{1}\right) \text {. }
$$

According to their definition the quantities $B_{j}^{u, t}$ are real for $u+t-J=$ even, and imaginary for $u+t-J=$ odd.

(f) For the angular distribution of electrons from oriented nuclei: $\left(\theta_{e}=\right.$ angle between axis of alignment and $\beta$-momentum),

With

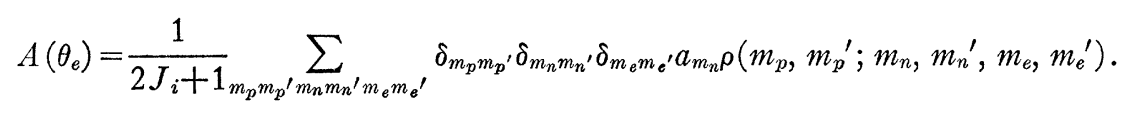

it becomes

$$
h_{J}\left(J_{i}\right)=\sum_{m_{n}} a_{m_{n}}(-)^{J_{i}-m_{n}} \frac{1}{\left(2 J_{i}+1\right)^{\frac{1}{2}}} c\left(J_{i} J_{i} J ; m_{n},-m_{n}\right)
$$

$$
A\left(\theta_{e}\right)=16 \pi \sum_{K K^{\prime} J} h_{J}\left(J_{i}\right)(-)^{K+K^{\prime}+J} F_{J^{0}}^{0, J}\left(K, K^{\prime} ; J_{f}, J_{i}\right) B_{J}^{J, 0}\left(K, K^{\prime} ; J_{f}, J_{i}\right) P_{J^{0}}\left(\theta_{e}\right) .
$$

(g) For the angular distribution of $\gamma$ rays following the $\beta$ decay of oriented nuclei:

$$
B\left(\theta_{\gamma}\right)=\frac{1}{2 J_{i}+1} \sum_{m_{p} m_{p^{\prime}} m_{n} m_{n^{\prime}} m_{e} m_{e}{ }^{\prime} \tau} a_{m_{n}} \delta_{m_{p} m_{p}} \delta_{m_{n} m_{n}{ }^{\prime}} \delta_{m_{e} m_{e^{\prime}}} \int \rho\left(m_{p}, m_{p}{ }^{\prime} ; m_{n}, m_{n}{ }^{\prime} ; m_{e}, m_{e}{ }^{\prime}\right) d \Omega_{e} \rho_{\gamma}\left(m_{p}, m_{p}^{\prime} ; \tau\right)
$$

or, with the same definition of the quantities $h_{J}\left(J_{i}\right)$ as above,

$$
\begin{array}{r}
B\left(\theta_{\gamma}\right)=128 \pi^{2} \sum_{K K^{\prime} \lambda \lambda^{\prime} J} \delta_{\lambda} \delta_{\lambda^{\prime}} B_{0}^{0,0}\left(K, K^{\prime} ; J_{f}, J_{i}\right) F_{0}^{J, J}\left(K, K^{\prime} ; J_{f}, J_{i}\right) h_{J}\left(J_{i}\right) F_{J^{0}}^{0, J}\left(\lambda, \lambda^{\prime} ; J_{f f}, J_{f}\right) \\
\cdot \frac{(-)^{K+K^{\prime}+J}}{\left[\left(2 J_{f}+1\right)(2 J+1)\right]^{\frac{1}{2}}} P_{J}\left(\theta_{\gamma}\right) .
\end{array}
$$

For the application of these formulas, one should also make use of the relations

$$
(-)^{K+K^{\prime}} F_{J}{ }^{J, 0}\left(K, K^{\prime} ; J_{f}, J_{i}\right)=F_{J}{ }^{0, J}\left(K, K^{\prime} ; J_{i}, J_{f}\right)=F_{J}\left(K, K^{\prime} ; J_{i}, J_{f}\right)
$$

where $F_{J}\left(K, K^{\prime} ; J_{i}, J_{f}\right)$ is the quantity tabulated in the literature (ALD 57 , FE 55 ); of Table III or IV, and of Table VI for the quantities $F_{0}{ }^{J J}\left(K, K^{\prime} ; J_{i}, J_{f}\right)$. 


\section{APPENDIX V. CALCULATIONS OF THE REDUCED LEP- TON MATRIX ELEMENTS. EXPRESSIONS FOR THE BJ'S IN TERMS OF REDUCED NUCLEAR MATRIX ELEMENTS AND ELECTRON AND NEUTRINO RADIAL WAVE FUNCTIONS}

We now derive an expression for the $B_{J}$ 's where they appear as a simple function of the reduced nuclear matrix elements involved and the electron and neutrino radial wave functions $f_{\kappa}(k R), g_{\kappa}(k R)$ and $j_{l(k)}(q R)$.

For this purpose we first calculate the reduced lepton matrix elements $\left\langle j\left(\kappa_{e}\right)\left\|\left(1+\gamma_{5}\right) T_{K L \gamma}\right\| j\left(\kappa_{v}\right)\right\rangle$. These matrix elements depend on $r$. According to Eq. (C8a), they have to be multiplied with the nuclear matrix elements, also functions of $r$, and integrated over $r$.

We use the definition (C6) of the reduced matrix elements, the definition $(\mathrm{C} 2 \mathrm{~b})$ of the tensor operators $T_{K L \gamma}$ and the definitions (A11) and (C4a) of the negaton and neutrino wave function. A straightforward application of Racah techniques yields

$$
\begin{aligned}
& (4 \pi)^{\frac{1}{2}} \cdot\left\langle j\left(\kappa_{e}\right)\left\|\left(1+\gamma_{5}\right) T_{K L \gamma}\right\| j\left(\kappa_{\nu}\right)\right\rangle=f_{\kappa_{e}}{ }^{*} j_{l}\left(\kappa_{\nu}\right) G_{K L \gamma}\left(\kappa_{e}, \kappa_{\nu}\right)+\operatorname{Sg} \kappa_{e} g_{\kappa_{e}}{ }^{*} j_{l}\left(\kappa_{\nu}\right) G_{K L \gamma}\left(-\kappa_{e},+\kappa_{\nu}\right) \\
& +f_{\kappa_{e}}{ }^{*} j_{l}\left(-\kappa_{\nu}\right) G_{K L \gamma}\left(\kappa_{e},-\kappa_{\nu}\right)+\operatorname{Sg} \kappa_{e} g_{\kappa_{e}}{ }^{*} j_{l}\left(-\kappa_{\nu}\right) G_{K L \gamma}\left(-\kappa_{e},-\kappa_{\nu}\right) \text {. } \\
& G_{K L \gamma}\left(\kappa_{e}, \kappa_{\nu}\right)=\left\{(2 \gamma+1)(2 K+1)\left[2 l\left(\kappa_{e}\right)+1\right]\left[2 l\left(\kappa_{\nu}\right)+1\right]\left[2 j\left(\kappa_{e}\right)+1\right]\left[2 j\left(\kappa_{\nu}\right)+1\right]\right\}^{\frac{1}{2}} i^{l\left(\kappa_{\nu}\right)+L-l\left(\kappa_{e}\right)} c\left(l\left(\kappa_{e}\right), l\left(\kappa_{\nu}\right), L ; 00\right) \\
& \cdot(-)^{j\left(\kappa_{\nu}\right)-j\left(\kappa_{e}\right)+l\left(\kappa_{e}\right)} \times\left\{\begin{array}{ccc}
K & \gamma & L \\
j\left(\kappa_{e}\right) & \frac{1}{2} & l\left(\kappa_{e}\right) \\
j\left(\kappa_{\nu}\right) & \frac{1}{2} & l\left(\kappa_{\nu}\right)
\end{array}\right\} .
\end{aligned}
$$

As a next step, we connect the definition of the reduced nuclear matrix elements with their definition in cartesian coordinates by means of the formula

$$
\left\langle J_{f}\left\|\left(C_{V}-C_{A} \gamma_{5}\right) T_{K \gamma L}\right\| J_{i}\right\rangle=\left(2 J_{i}+1\right)^{\frac{1}{2}}\left\langle J_{f}\left\|\left(C_{V}-C_{A} \gamma_{5}\right) T_{\text {cart }}\right\| J_{i}\right\rangle
$$

Table II gives an explicit representation of the relation (E3) for allowed and first-forbidden operators. This table can be calculated easily by using explicit formulas for the Clebsch-Gordan coefficients involved in the definition of the $T_{K L \gamma}$, and by using only the 0 component of the operator, which has to be compared with the $z$ component of the corresponding cartesian operator.

By combining Table II, and Eqs. (C9a), (E1), and (E2), we can express the quantities $B_{J}{ }^{u t}$ as a simple bilinear form in the expressions of the form

$$
\begin{gathered}
\int r^{2} d r\left[\left\langle J_{f}\left\|C_{V} T_{\text {cart }}\right\| J_{i}\right\rangle_{r_{n}=r} f_{\kappa_{e}}(r) j_{l(k v)}(r)\right], \text { etc., and } \\
\int r^{2} d r\left[\left\langle J_{f}\left\|C_{A} \gamma_{5} T_{\text {cart }}\right\| J_{i}\right\rangle_{r_{n}=r} f_{\kappa_{e}}(r) j_{l(k v)}(r)\right], \text { etc. }
\end{gathered}
$$

For the most important cases occurring in Eqs. (D2) to (D8) of Appendix IV, this has been done explicitly in Table III. The actual calculation of Table III is quite cumbersome for some of the cases since it involves the explicit evaluation of the $6 j-$ and $9 j-$ symbols used in Eqs. (C9a) and (E2).

Since the definition of the $B_{J^{u t}}$ involves an infinite sum over the neutrino and electron spins and momenta, the following further approximation has been made in the calculation of Table III:

In the case of allowed transitions, only terms of zeroth (lowest) power in the neutrino momentum have been kept, i.e., only terms multiplied by $j_{0}(q R)$, where we have put $j_{0}(q R)=1$. Also the electron spin has been limited to $j_{e}=\frac{1}{2}$ or $\kappa_{e}= \pm 1$. This is because Eq. (A15) shows that $f_{\kappa}, g_{\kappa} \approx(k r)^{\left[x^{2}-Z \alpha^{2}\right]-1}$.

A similar approximation has been used in the case of first-forbidden transitions.
The remaining radial integrals do not occur as such explicitly in Table III. For instance, the expression $\left|\int 1\right|^{2} L$ is supposed to mean

$$
\begin{aligned}
\left|\int\left\langle J_{f}\|1\| J_{i}\right\rangle_{r} g_{1}(k r) r^{2} d r\right|^{2} & \\
& +\left|\int\left\langle J_{f}\|1\| J_{i}\right\rangle_{r} f_{-1}(k r) r^{2} d r\right|^{2}
\end{aligned}
$$

and correspondingly for the other expressions. Since in this form the radial integrals still contain the dependence on the energy of the electron, further approximations are required to make this dependence explicit. This is discussed in the next appendix where the various possible approximations, taking into account screening and finite nuclear size, are discussed, and the reduction of the expressions occurring in Table III to a simple form for various possible approximations is given. 
TABLE VI. Coefficients $F_{0}{ }^{J}\left(K, K ; J_{1}, J_{2}\right)$ for some important cases. ${ }^{a}$ Note:

$F_{0}^{J J}\left(K, K ; J_{1}, J_{2}\right) \equiv F_{0}^{J J}\left(K, K ; J_{2}, J_{1}\right) ;$

$F_{0}{ }^{00}\left(K, K ; J_{2}, J_{1}\right)=1$.

(1) $K=0, F_{0}^{J J}\left(0,0 ; J_{1}, J_{2}\right)=\delta_{J_{1} J_{2}}(2 J+1)^{\frac{1}{2}}$

(2) $K=1, J_{1}$ is an integer.

\begin{tabular}{cccccc}
\hline \hline & \multicolumn{1}{c}{$J$} & & & \\
$J_{1} J_{2}$ & & 1 & 2 & 3 & 4 \\
\hline 1 & 0 & 0 & 0 & 0 & 0 \\
1 & 1 & +0.866 & -1.118 & 0 & 0 \\
2 & 1 & +1.500 & +1.323 & 0 & 0 \\
2 & 2 & +1.443 & +1.118 & 0 & -2.000 \\
3 & 2 & +1.633 & +1.852 & +1.732 & +1.254 \\
3 & 3 & +1.588 & +1.677 & +1.323 & +0.500 \\
4 & 3 & +1.677 & +2.023 & +2.141 & +2.044 \\
4 & 4 & +1.645 & +1.901 & +1.852 & +1.500 \\
5 & 4 & +1.697 & +2.101 & +2.325 & +2.393 \\
5 & 5 & +1.674 & +2.012 & +2.117 & +2.000 \\
6 & 5 & +1.708 & +2.142 & +2.424 & +2.580
\end{tabular}

(3) $K=1, J_{1}$ is a half integer.

\begin{tabular}{lllccc}
$1 / 2$ & $1 / 2$ & -0.577 & 0 & 0 & 0 \\
$3 / 2$ & $1 / 2$ & +1.291 & 0 & 0 & 0 \\
$3 / 2$ & $3 / 2$ & +1.270 & +0.447 & -1.587 & 0 \\
$5 / 2$ & $3 / 2$ & +1.587 & +1.673 & +1.296 & 0 \\
$5 / 2$ & $5 / 2$ & +1.534 & +1.469 & +0.832 & -0.429 \\
$7 / 2$ & $5 / 2$ & +1.660 & +1.956 & +1.982 & +1.741 \\
$7 / 2$ & $7 / 2$ & +1.622 & +1.810 & +1.638 & +1.095 \\
$9 / 2$ & $7 / 2$ & +1.689 & +2.068 & +2.248 & +2.248 \\
$9 / 2$ & $9 / 2$ & +1.662 & +1.965 & +2.004 & +1.788 \\
$11 / 29 / 2$ & +1.703 & +2.124 & +2.381 & +2.500 \\
\hline
\end{tabular}

(4) When $K=2$, in case it should ever be needed, $F$ is given by

$F_{0}^{J J}\left(3,2 ; J_{1}, J_{2}\right)=(-)^{-J+J_{1}+J_{2}}$

$\cdot\left[(2 J+1)\left(2 J_{1}+1\right)\left(2 J_{2}+1\right)\right]^{\frac{1}{2}}$

-W( $\left.J_{1} J_{2} J_{1} J_{2} ; 2 J\right)$

a For $K=0, F$ as defined in $\left(\mathrm{C}_{9} \mathrm{c}\right)$ is actually zero. Therefore, we have changed the definition so that the product $F_{0}{ }^{J J}\left(K, K ; J_{f}, J_{i}\right) B_{0} 00\left(K, K ; J_{f}, J_{i}\right)$ has the right value.

With the help of Eqs. (D2) to (D8), Table III, and the tabulated values for the coefficients $F_{J}{ }^{u t}$, we can now write all the measurable quantities as functions of the reduced nuclear matrix elements.

\section{APPENDIX VI. KONOPINSKI-UHLENBECK APPROXIMATION. INFLUENCE OF SCREENING AND FINITE NUCLEAR SIZE}

The expressions derived in Appendix $\mathrm{V}$ do not show their dependence on the energy of the beta particle.
Therefore, one expands the functions $f_{k}$ and $g_{k}$ into powers of $\boldsymbol{r}$ and keeps only the first term. This way the energy dependence becomes explicit.

The power-series expansion of $f_{\kappa}$ and $g_{\kappa}$ was given in Appendix I for the case of a point nucleus. In many applications, this is a bad approximation to the actual wave function, which corresponds to an extended nuclear charge distribution, and which is also influenced by the fact that the outgoing electron sees a screened nuclear charge distribution once its distance from the nucleus becomes as large as a few Bohr radii.

This appendix describes the various approximations used in the literature.

\section{A. Konopinski-Uhlenbeck Approximation (KON 41)}

One uses the wave functions for a point nucleus as given in Appendix I, and expands them and the expressions $L_{v}, M_{v}$, etc., occurring in Table III in powers of $r$, keeping only the first term in the expansion. According to Eq. (A15), this first term is proportional to $(k r)^{\gamma-1}$, where $\gamma=\left[\kappa^{2}-(Z \alpha)^{2}\right]^{\frac{1}{2}}$

$$
f_{\kappa}, g_{\kappa} \sim(k r)^{\gamma-1}+\text { higher powers. }
$$

This $r$-dependent factor actually is part of the nuclear matrix elements because we have to integrate over $r$. Therefore, one would arrive at expressions of the form

$$
\int r^{\gamma-1} d \tau, \int d r^{\gamma-1} d \tau
$$

etc., for allowed, and at corresponding expressions for forbidden transitions. The usual approximation consists of writing

$$
\int r^{\gamma-1} d \tau=R^{\gamma-1} \int 1 d \tau ; \int \delta r^{\gamma-1} d \tau=R^{\gamma-1} \int \delta d \tau
$$

etc. Here, $R$ denotes the nuclear radius, and $R^{\gamma-1}$ is subsumed into the Fermi function $F(Z, E)$, whichbeing obtained from a bilinear expression in the matrix elements-contains a factor $R^{2 \gamma_{1}-2}$, where $\gamma_{1}=$ $\left[1-(Z \alpha)^{2}\right]^{\frac{1}{2}}$. This procedure together with the approximation $\left[1-(Z \alpha)^{2}\right]^{\frac{1}{2}}=1-\frac{1}{2}(Z \alpha)^{2}$ and a similar approximation for the $\Gamma$ function forms the basic assumption of the Konopinski-Uhlenbeck approximation, with the help of which all the expressions in Table IV can be obtained from the expressions in Table III. Values for the Fermi function $F(Z, E)$ and related quantities have been tabulated in the point nucleus approximation by various authors (DA 51; FA 52; LA 54; MAC 52; MOS 51; WA 59). In particular, Kotani and Ross (KOT 59a) give a fairly complete tabulation of the relevant functions for first-forbidden decay, without making the approximation $(Z \alpha)^{2} \ll 1$. It turns out, that the changes due to Coulomb corrections may become quite appreciable. One has to realize that $\left[1-(Z \alpha)^{2}\right]^{\frac{1}{2}}-$ $1<0$, and that, therefore, for $\kappa= \pm 1$ the functions $f_{k}, g_{k}$ are actually infinite at the center of the nucleus. By application of Eqs. (F3) this difficulty has been circumvented. 


\section{B. Finite Nuclear Size Effects and Screening}

With a smeared-out nuclear charge distribution, such a difficulty does not exist any more, since all functions $f_{k}, g_{k}$ are regular in the origin. One still uses the same scheme, however; that is, one expands the functions $f_{\kappa}, g_{\kappa}$ into powers of $r$ and takes the leading term. (The variation of the wave function over the nuclear volume is very small.) Then one applies Eq. (F3) or equations similar in spirit, and obtains the explicit energy dependence of the measurable quantities. This method of using only the terms of lowest order in $r$ seems generally justified. It is done in the spirit of the "forbiddenness approximation," where higher powers in $(k r)$ correspond to a higher degree of forbiddenness, and correspond to the neglect of retardation terms in the electromagnetic case. It has been pointed out, however, that there might be cases as, for example, in $\mathrm{RaE}$, where-due to a cancellation of the leading terms-this approximation breaks down, and one has to take into account terms of the type neglected here (YA 53d; see also Sec. 7, RaE). These terms might be called "finite size terms" in contrast to genuine "third-orderforbidden" corrections. In the following, we discuss only the leading terms in $(k r)$.

Therefore, we want the leading term in the powerseries expansion of $F_{\kappa}=r f_{\kappa}, G_{\kappa}=r g_{\kappa}$, where $F_{\kappa}$ and $G_{\kappa}$ obey the Eqs. (A14)

$$
\begin{aligned}
& d / d r F_{\kappa}=-\frac{\kappa}{r} F_{\kappa}+(E+1-V) G_{\kappa}, \\
& d / d r G_{\kappa}=-(E-1-V) F_{\kappa}+{ }_{r}^{\kappa} G_{\kappa},
\end{aligned}
$$

as in Appendix I. Here, $V$ is no longer the Coulomb potential $-Z e^{2} / r$ but a modified potential taking into account finite nuclear size and screening. This problem has been treated by various authors (BEN 58; BR 58, DO 56; DZ 56; GE 58c; LO 49a, LO 49b, LO 49c; KOT 58b; MAL 51, MAL 52; NA 54; RO 51, RO 53a, RO 53b; YA 53b, YA 53d; ZY 56); for numerical values, see also (SL 56). ${ }^{12}$ We give a brief outline of the procedures and results.

We first consider the effects of screening: because of screening, the potential $V(r)$ becomes zero outside the atom (this causes the logarithmic phase shifts in Appendix I to disappear). Inside the atom, the potential tends towards zero faster than in the point-nucleus case without screening. Inside the nucleus, the potential is essentially unchanged. Since we are interested only in the behavior of the wave functions inside the nucleus, screening therefore leaves only one effect; it changes the normalization of the wave functions by a few percent at low energies, and has hardly any effect at all at higher energies ("screening factor").

12 According to the appendix of Bühring (BU 59), there is an error in these tables. Since the tables were not available, however, the present author was not able to check this statement.
The "finite size cffect," i.e., the change of the first terms in the power-series expansion of $f_{\mathrm{k}}$ and $g_{\kappa}$, becomes less important with increasing $\kappa$ and decreasing $Z$; in fact, according to Rose and Holmes (RO 51), it can be neglected altogether for $Z \leq 60$ and $|\kappa| \geq 3$.

The effect of a change of the potential in Eq. (F4) on the wave functions $F$ and $G$ can be discussed according to Rose and Holmes (RO 51): The shape of $F_{\kappa}$ and $G_{\kappa}$ is determined by the orbital angular momentum $l(\kappa)$ and by the shape of the potential $V(r)$. A formal solution of the Eqs. (F4) can be obtained by integration if $\lim _{r \rightarrow 0}(r V)=0$, one has

$$
\begin{aligned}
& F_{\kappa}=r^{-\kappa}\left[C_{1}+\int_{0}^{r_{0}} r^{\kappa}(E+1-V) G_{\kappa} d r\right], \\
& G_{\kappa}=r^{\kappa}\left[C_{2}+\int_{0}^{r} r^{-\kappa}(-E+1+V) F_{\kappa} d r\right],
\end{aligned}
$$

and for solutions regular in the origin, $C_{1}=0$ for $\kappa>0$, $C_{2}=0$ for $\kappa<0$.

This shows, that for $\kappa>0, F_{\kappa}$ is sensitive to changes in the potential in the first order, whereas the leading term in $G_{\mathrm{k}}$ is determined by $r^{\kappa} C_{2}$, and therefore determined by the angular momentum. Of course, also $C_{2}$ is subject to change (the potential corresponding to finite nuclear size is less attractive inside the nucleus than a point-nucleus Coulomb potential, and therefore a smaller fraction of the wave function is inside the nuclear volume), but this change is very slight and of higher order in the change of potential. The opposite is true for $\kappa<0$, and the conclusion is that quantities depending on $f_{\kappa}$ for $\kappa>0$ and on $g_{\kappa}$ for $\kappa<0$ will be most strongly influenced. Tables III and IV show that the changes in spectrum, for example, for the $\xi$ approximation and the unique forbidden transitions are small; the strongest influence is to be expected for the other transitions-the ones, in fact, in which we are mainly interested.

The order of magnitude of the changes depends, of course, on the energy $W$ as well as on $Z$. For the spectrum, the changes turn out to become as large as $20 \%$; they are, however, fairly insensitive to a change in energy, which preserves, for example, the character of an allowed spectrum. However, in evaluating $\log -\mathrm{ft}$ values, such effects have to be included.

\section{APPENDIX VII. HOW TO CHANGE FORMULAS FOR CASES OF ORBITAL ELECTRON CAPTURE AND POSITRON DECAY}

We now derive the rules for how to change the formulas for orbital electron capture and $\beta^{+}$emission.

We start with orbital electron capture and remember that-opposite to the case of $\beta^{-}$emission - a neutrino (not an antineutrino) is liberated and an electron destroyed. That means, that for this process the hermitian conjugate of the operator for $\beta^{-}$emission is 
responsible

$$
\begin{aligned}
H_{\text {o.c. }}=\left(H_{\beta^{-}}\right)+=\left[\left(\psi_{p} \gamma_{4} \gamma_{\mu}\left(C_{V}-C_{A} \gamma_{5}\right) \psi_{n}\right]\right. & \\
\cdot\left[\psi_{e}{ }^{*} \gamma_{4} \gamma_{\mu}\left(1+\gamma_{5}\right) \psi_{\nu}\right]^{+}= & {\left[\psi_{n}{ }^{*}\left(C_{V}-C_{A} \gamma_{5}\right) \gamma_{\mu} \gamma_{4} \psi_{p}\right] } \\
& \cdot\left[\psi_{\nu}{ }^{*}\left(1+\gamma_{5}\right) \gamma_{\mu} \gamma_{4} \psi_{e}\right] .
\end{aligned}
$$

We rewrite this operator in such a way that it resembles $H_{\beta^{-}}$in the following sense: It is obtained from $H_{\beta^{-}}$by making natural replacements corresponding to the transition from $\beta^{-}$emission to the orbital electron capture, namely replacing the neutron by the proton wave function and vice versa and the electron by the neutrino function and vice versa. It is clear that this is the proper replacement, since $\beta^{-}$emission is described by the reaction

$$
\nu+n \rightarrow p+\beta^{-}
$$

and orbital electron capture by

$$
p+\beta^{-} \rightarrow \nu+n \text {. }
$$

Now, Eq. (G1) becomes

$$
H_{\text {o.c. }}=\left[\psi_{n}{ }^{*} \gamma_{4} \gamma_{\mu}\left(C_{V}-C_{A} \gamma_{5}\right) \psi_{p}\right]\left[\psi_{\nu}{ }^{*} \gamma_{4} \gamma_{\mu}\left(1+\gamma_{5}\right) \psi_{e}\right] \text {; }
$$

that is, it has exactly the form of $H_{\beta^{-}}$if one makes the replacements indicated by Eqs. (G2a) and (G2b). In the language of parity conserving and nonconserving coupling constants this means $C_{A} \rightarrow C_{A}, C_{V} \rightarrow C_{V}$, $C_{A}{ }^{\prime} \rightarrow C_{A}{ }^{\prime}, C_{V^{\prime}} \rightarrow C_{V^{\prime}}{ }^{\prime}$, or-none of our formulas has to be changed (except that we have to use the right energy and bound state wave function for the captured electron; for the case of $K$ capture there is only a contribution from the bound state wave function $\chi=1$, etc.) [see (BR 58)].

For the case of $\beta^{+}$emission, we proceed similarly, except that here the proper replacement is: Proton wave function is replaced by neutron wave function and vice versa, electron wave function is replaced by positron wave function, and neutrino wave function is replaced by antineutrino wave function. This holds true because the reaction can be described by

$$
p+\bar{\nu} \rightarrow n+\beta^{+} \text {. }
$$

Again we have to use the hermitian conjugate of the $\beta^{-}$-emission operator. Since here, however, some of the coupling constants will change, we prefer to write things somewhat more explicitly:

$$
\begin{aligned}
& H_{\beta^{+}}=\left(H_{\beta^{-}}\right)^{+}=\left\{\left(\psi_{p}^{*} \gamma_{4} \gamma_{\mu} \psi_{n}\right)\left[\psi_{e}^{*} \gamma_{4} \gamma_{\mu}\left(C_{V}+C_{V^{\prime}} \gamma_{5}\right) \psi_{\nu}\right]\right. \\
& \left.\quad+\left(\psi_{p}^{*} \gamma_{4} \gamma_{\mu} \gamma_{5} \psi_{n}\right)\left[\psi_{e}{ }^{*} \gamma_{4} \gamma_{\mu} \gamma_{5}\left(C_{A}+C_{A} \gamma_{5}\right) \psi_{\nu}\right]\right\}^{+} .
\end{aligned}
$$

This is equal to

$$
\begin{aligned}
H_{\beta^{+}}= & \left(\psi_{n}{ }^{*} \gamma_{4} \gamma_{\mu} \psi_{p}\right)\left[\psi_{\nu}{ }^{*} \gamma_{4} \gamma_{\mu}\left(C_{V}+C_{V}{ }^{\prime} \gamma_{5}\right) \psi_{e}\right] \\
& +\left(\psi_{n}{ }^{*} \gamma_{4} \gamma_{\mu} \gamma_{5} \psi_{p}\right)\left[\psi_{\nu}{ }^{*} \gamma_{4} \gamma_{\mu} \gamma_{5}\left(C_{A}+C_{A}{ }^{\prime} \gamma_{5}\right) \psi_{e}\right] .
\end{aligned}
$$

In order to replace the wave function $\psi_{e}$, which destroys an electron, by $\psi_{\bar{e}}^{*}$, the wave function which creates a positron, and $\psi_{\nu}{ }^{*}$, which creates a neutrino, by $\psi_{\bar{v}}$, which destroys an antineutrino, we recall that the charge conjugation operator $C$ has the following properties:

$$
\begin{aligned}
& C C^{*}=1, \\
& C^{* \mathrm{~T}} \gamma_{i}{ }^{\mathrm{T}}=\gamma_{i} C^{*}, \text { where } i=1,2,3, \\
& \text { and T means "transposed," } \\
& C^{* \mathrm{~T}}{\gamma_{4}}^{\mathrm{T}}=-\gamma_{4} C^{*}, \\
& C \psi_{e}(-E, Z)=\psi_{\bar{e}}{ }^{*}(E,-Z), \\
& C \psi_{\nu}(+E)=\psi_{\bar{\nu}}{ }^{*}(-E) .
\end{aligned}
$$

Inserting $C^{*} C=1$ in front of $\psi_{e}$, and using Eq. (G7), $H_{\beta}+$ takes the form

$$
\begin{aligned}
& H_{\beta^{+}}=\left(\psi_{n}{ }^{*} \gamma_{4} \gamma_{\mu} \psi_{p}\right)\left[\psi_{\bar{e}}{ }^{*} \gamma_{4} \gamma_{\mu}\left(C_{V}-C_{V}{ }^{\prime} \gamma_{5}\right) \psi_{\bar{\nu}}\right] \\
& \quad+\left(\psi_{n}{ }^{*} \gamma_{4} \gamma_{\mu} \gamma_{5} \psi_{p}\right)\left[\psi_{\bar{e}}{ }^{*} \gamma_{4} \gamma_{\mu} \gamma_{5}\left(-C_{A}+C_{A}{ }^{\prime} \gamma_{5}\right) \psi_{\bar{\nu}}\right]
\end{aligned}
$$

This means that in the case of positron decay the following replacements have to be made:

$$
\begin{array}{ll}
C_{V} \rightarrow C_{V} & C_{V^{\prime}} \rightarrow-C_{V^{\prime}} \\
C_{A} \rightarrow-C_{A} & C_{A}{ }^{\prime} \rightarrow C_{A}{ }^{\prime} \\
Z \rightarrow-Z &
\end{array}
$$

Since we have not written our results in terms of the primed and unprimed coupling constants, the following rule holds: In quantities which are not due to parity nonconservation, i.e., spectrum shape, $\beta-\gamma$ angular correlation, etc., no interference terms between unprimed and primed coupling constants enter. Here, therefore, the rule $C_{V} \rightarrow C_{V}, C_{A} \rightarrow-C_{A}, Z \rightarrow-Z$, holds.

Quantities which are due to parity nonconservation, such as electron polarization, $\beta$ circularly polarized $\gamma$-angular correlation, etc., depend always on either one of the interference terms $C_{V} C_{V}{ }^{\prime}, C_{A} C_{A}{ }^{\prime}, C_{V} C_{A}{ }^{\prime}$ or $C_{A} C_{V}{ }^{\prime}$. Therefore, the rule is here: Quantities proportional to $C_{V}^{2}$ or $C_{A}{ }^{2}$ change sign, quantities proportional to $C_{V} C_{A}$ remain unchanged, and $Z$ must be replaced by $-Z$. This can be interpreted very easily: Whereas for $\beta^{-}$emission the right coupling is given by $V-A$, for $\beta^{+}$emission this coupling reads $V+A$, and the spins of the $\beta^{-}$and $\beta^{+}$particles have opposite direction.

\section{APPENDIX VIII. SOME DEFINITIONS}

We define the spherical harmonics in the usual way: For $m=l, l-1, \cdots, 0$ we put (EL 57 )

$$
\begin{aligned}
Y_{l}^{m}=e^{i m \varphi} \frac{(-)^{l+m}}{(2 l) ! !}\left[\frac{(2 l+1)}{4 \pi} \frac{(l-m) !}{(l+m) !}\right]^{\frac{1}{2}}(\sin \theta)^{m} \\
\\
\frac{d^{l+m}}{d(\cos \theta)^{l+m}}(\sin \theta)^{2 l} .
\end{aligned}
$$

For $m=-1, \cdots,-l$, we have $Y_{l^{m}}=(-)^{m} Y_{l}^{*-m}$ (H1a) 
The spherical harmonics transform under rotations according to the formula

$$
Y_{e}^{m}\left(\vartheta^{\prime}, \varphi^{\prime}\right)=\sum_{\mu} D_{l^{\mu m}}\left(\theta_{i}\right) Y_{e}^{\mu}(\vartheta, \varphi)
$$

where $\theta_{i}$ are the Euler angles of the rotation. Particularly, we have

$$
D_{l}^{m 0}(\alpha \beta \gamma)=[4 \pi /(2 l+1)]^{\frac{1}{2}} \cdot(-)^{m} Y_{l^{m}}(\beta, \alpha) .
$$

The Clebsch-Gordan coefficients are defined as in Condon and Shortley, (CO 35); the theorem by Clebsch and Gordan takes the form

$$
\psi_{j_{1}}{ }^{m_{1}} \psi_{j_{2}}{ }^{m_{2}}=\sum_{j} c\left(j_{1} j_{2} j ; m_{1} m_{2}\right) \psi_{j}^{m_{1}+m_{2}}
$$

if $\psi_{j}{ }^{\mu}$ spans the irreducible representation of degree $2 j+1$ of the rotational group.

The Wigner 3- $j$ coefficients are related to the ClebschGordan coefficients by (EL 57)

$c\left(j_{1} j_{2} j_{3} ; m_{1}, m_{2}\right)=(-)^{j_{1}-j_{2}+m_{1}+m_{2}}\left[2 j_{3}+1\right]^{\frac{1}{2}}$

$$
\left(\begin{array}{ccc}
j_{1} & j_{2} & j_{3} \\
m_{1} & m_{2} & \left(-m_{1}-m_{2}\right)
\end{array}\right) .
$$

The Wigner 3-j coefficient has the following symmetry properties: An even permutation of the columns causes no change in value, while an odd permutation introduces a factor $(-)^{j_{1}+j_{2}+j_{3}}$.

The Racah coefficients are defined by (RO 55)

$$
\begin{aligned}
& {\left[(2 j+1)\left(2 j^{\prime}+1\right)\right]^{\frac{1}{2}} W\left(j_{1} j_{2} j_{4} j_{3} ; j, j^{\prime}\right)=\sum_{m_{1} m_{2} m_{3} m_{4} m m^{\prime}}} \\
& \quad \cdot c\left(j, j_{3}, j_{4} ; m, m_{3}\right) c\left(j_{1}, j_{2}, j ; m_{1}, m_{2}\right) c\left(j_{2} j_{3} j^{\prime} ; m_{2}, m_{3}\right) \\
& \quad \cdot c\left(j_{1} j^{\prime} j_{4} ; m_{1}, m^{\prime}\right) \delta_{m+m_{3}, m_{4}} \delta_{m_{1}+m_{2}, m} \delta_{m_{2}+m_{3}, m^{\prime}} \delta_{m_{1}+m^{\prime}, m_{4}} \cdot
\end{aligned}
$$

They are related to the Wigner $6-j$ symbol by (EL 57 )

$$
W\left(j_{1}, j_{2}, j_{4}, j_{3} ; j, j^{\prime}\right)=(-)^{j_{1}+j_{2}+j_{3}+j_{4}}\left\{\begin{array}{ccc}
j_{1} & j_{2} & j \\
j_{3} & j_{4} & j^{\prime}
\end{array}\right\} \text {. }
$$

The 6- $j$ symbol has the following symmetry properties: Any two columns may be interchanged without changing its value, and any two elements of the top row may be interchanged with those directly beneath them without interchanging its value.

The $9-j$ symbol is defined by

$$
\begin{aligned}
\left\{\begin{array}{lll}
a & f & j \\
d & h & e \\
g & c & b
\end{array}\right\}=(-)^{2 a+2 b} \sum_{x}(2 x+1) & \cdot\left\{\begin{array}{lll}
a & b & x \\
c & d & g
\end{array}\right\}\left\{\begin{array}{lll}
c & d & x \\
e & f & h
\end{array}\right\}\left\{\begin{array}{lll}
e & f & x \\
a & b & j
\end{array}\right\} .
\end{aligned}
$$

Finally the $D$ functions fulfill the relationships

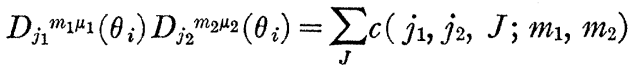

$$
\begin{aligned}
& \cdot c\left(j_{1}, j_{2}, J ; \mu_{1}, \mu_{2}\right) D_{J}^{m_{1}+m_{2}, \mu_{1}+\mu_{2}}\left(\theta_{i}\right) \\
& D_{j_{1}}{ }^{* m_{1} \mu_{1}}\left(\theta_{i}\right)=(-)^{m_{1}-\mu_{1}} D_{j_{1}}-m_{1}-\mu_{1}\left(\theta_{i}\right) \text {. }
\end{aligned}
$$

Our $\gamma$ matrices and Dirac equation are defined in the following way:

$$
\left.\begin{array}{l}
\boldsymbol{\delta} \gamma_{5}=\alpha,[\boldsymbol{\alpha}, \beta]=2 \boldsymbol{\alpha} \beta,\left[\boldsymbol{\delta}, \gamma_{5}\right]=0=[\boldsymbol{\delta}, \beta] \\
\gamma_{i}=-i \beta \alpha_{i} ; i=1,2,3 ; \gamma_{4}=-\beta ; \gamma_{5}=\gamma_{1} \cdot \gamma_{2} \cdot \gamma_{3} \cdot \gamma_{4}
\end{array}\right\} .
$$

Since we use the Dirac equation in the form

$$
H=-\alpha \mathrm{p}-\beta m=i \partial / \partial t
$$

we can introduce the operator

$$
\partial_{\mu}=\left\{\partial / \partial x_{i}, \partial / \partial(i t)\right\}
$$

and use formally euclidean metric, i.e., $g_{i j}=\delta_{i j}=g^{i j}$. Thus we have from (H12)

$$
\left(\partial_{\mu} \gamma_{u}+m\right) \psi=0 .
$$

In the Appendixes I-VII, $m$ is always the electron mass and therefore equal to one.

\section{GENERAL REFERENCES}

The following papers contain general formulas important for the interpretation of $\beta$-decay experiments. The list includes papers on subjects like recoil experiments, internal bremsstrahlung, etc., which have not been covered in the text:

ALD 57; BER 58a, 58b, 58c; BI 58b; BL 51; BR 58; CU 57; DE 58a, 58b; DO 57, 58a, 58b, 59; DR 59; FA 50; FO 54; FR 57; GA 59; GR 51; IB 58; KON 41; KOT 58, 59a, 59b; KU 59a, 59b; LEE 58a, 58b; LEW 57b; MAH 52, 59; ME 51; MO 53a, 53b, 53c, 58a, 58b, 60; NA 50; SK 50; SM 51; SP 52a, 52b; TA $54 \mathrm{~b}$; YA 52a.

\section{BIBLIOGRAPHY}

AH $52 \mathrm{a}$ $\mathrm{AH} 52 \mathrm{~b}$

AH 53

AL 55a $\mathrm{Al} \mathrm{55b}$

AL 56

AL $57 \mathrm{a}$

Al $57 \mathrm{~b}$

Al $57 \mathrm{c}$

ALD 57
'T. Ahrens and E. Feenberg, Phys. Rev, 86, 64 (1952). T. Ahrens, E. Feenberg, and H. Primakoff, Phys. Rev. 87, 663 (1952).

'T. Ahrens, Phys. Rev. 90, 974 (1953).

G. Alaga, Phys. Rev. 100, 432 (1955).

G. Alaga, K. Alder, A. Bohr, and B. Mottelson, Kgl. Danske Videnskab. Selskab Mat.-fys. Medd. 29, 9 (1955).

G. Allaga, and B. Jakšić, Phys. Rev. 103, 1441 (1956).

G. Alaga, Nuclear Phys. 4, 625 (1957).

G. Alaga, L. Sips, and D. Tadic, Glasnik Mat. Fiz. i Astr. 12, 207 (1957).

G. Alaga, Glasnik Mat. Fiz. i Astr. 12, 245 (1957). 245 (1957).

K. Alder, B. Stech, and A. Winther, Phys. Rev. 107, 728 (1957). 
ALI 5

A. I. Alikhanow, G. P. Eliseyev, and V. A. Luibimov, Nuclear Phys. 13, 541 (1959).

BAN 60 P. Banerjee and H. D. Zeh, Z. Physik 159, 170 FO 54 (1960).

BA 60 R. K. Bardin, C. A. Barnes, W. A. Fowler, and P. A. FR 57 Seeger, Phys. Rev. Letters 5, 323 (1960).

BEN 53 P. Benoist-Gueutal, Ann. phys. 8, 593 (1953).

BER 58a V. B. Berestetsky, B. L. Joffe, A. P. Rudik, and K.

BER 58b V. B. Beresteksky, B. L. Joffe, A. P. Rudik, and K. A. Ter-Martirosyan, Nuclear Phys. 5, 464 (1958).

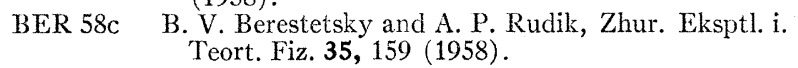

BH 60 C. P. Bhalla, Oak Ridge National Laboratory Rept: ORNL-2950 (1960).

BI 58a A. Bincer, E. Church, and J. Weneser, Phys. Rev. Letters 1, 95 (1958).

BI 58b A. M. Bincer, Phys. Rev. 112, 244 (1958).

BL 51 R. J. Blin-Stoyle and J. A. Spiers, Phys. Rev. 82, 969 (1951).

BR $51 \quad$ H. Brysk, Phys. Rev. 84, 362 (1951).

BR 53 H. Brysk, Phys. Rev. 90, 365 (1953)

BR 58 H. Brysk and M. E. Rose, Revs. Modern Phys. 30 , 1169 (1958).

BU 58a W. Bühring and J. Heintze, Phys. Rev. Letters 1, 176 (1958).

BU 58b W. Bühring and J. Heintze, Z. Physik 153, 237 (1958).

BU 59 W. Bühring, Z. Physik 155, 566 (1959).

CO 60 S. G. Cohen and R. Wiener, Nuclear Phys. 15, 79 (1960).

CO 35 E. U. Condon and G. H. Shortley, Theory of Atomic Spectra, (Cambridge University Press, Cambridge, England, 1935).

CO 58 J. M. Cork, M. K. Brice, R. G. Helmer, and R. M. Wood, Jr., Phys. Rev. 110, 526 (1958).

CU 57

R. B. Curtis and R. R. Lewis, Phys. Rev. 107, 543 (1957).

DA 51 J. P. Davidson, Phys. Rev. 82, 48 (1951).

DA 60 A. S. Davydov, Soviet Phys.-JETP 10, 98 (1960).

DE 58a A. Deloff, Bull. acad. polon. sci. Classe (III) 6, 1,57 (1958).

DE $58 \mathrm{~b}$ A. Deloff, Bull. acad. polon. sci. Classe (III) 6, 5,327 (1958)

DE 59 A. Deloff, Nuclear Phys. 13, 136 (1959).

DO 56 A. Z. Dolginov and I. N. Toptigin, Nuclear Phys. 2, 147 (1956).

Do 57 A. Z. Dolginov, Zhur. Eksptl. i. Teort. Fiz. 33, 1363 (1957).

DO 58a A. Z. Dolginov and N. P. Popov, Nuclear Phys. 7, $591(1958)$.

DO 58b A. Z. Dolginov, Nuclear Phys. 5, 512 (1958).

DO 59 A. Z. Dolginov and K. P. Popov, Soviet Phys.JETP 9, 368 (1959).

DR 59 J. F. Dreitlein, Phys. Rev. 116, 1604 (1959).

DZ 56 B. S. Dzhelepov and L. N. Zyrianowa, Influence of the Atomic Field on $\beta$ Decay, Academy of Sciences Press, U.S.S.R., 1956).

EL 57 J. P. Elliott and A. M. Lane, "The Nuclear Shell Model" in Handbuch der Physik (Springer-Verlag, Berlin, Germany, 1957), Vol. 39.

FA 50 D. L. Falkoff and G. E. Uhlenbeck, Phys. Rev. 79, $334(1950)$.

FA 52 U. Fano, Natl. Bureau Standards (U. S.) Appl. Math. Ser. 13, 61 (1952).
M. Ferentz and N. Rosenzweig, Argonne Natl. Lab. Rept. ANL-5324 (1955).

G. N. Fowler, Proc. Phys. Soc. (London) A67, 117 (1954).

H. Frauenfelder, A. D. Hanson, N. Levine, A. Rossi, and G. DePasquali, Phys. Rev. 107, 643 (1957).

GA 59

Y. V. Gaponov, Soviet Phys.-JETP 9, 131 (1959).

GEI 58

J. S. Geiger, G. T. Ewan, R. L. Graham, and D. R. MacKenzie, Phys. Rev. 112, 1684 (1958).

GE 58a

GE 58b

GE $58 \mathrm{c}$

B. V. Geškenbein, Nuovo cimento 10, 383 (1958).

B. V. Geškenbein, Zhur. Eksptl. i. Teort. Fiz. 34, 1349 (1958).

B. V. Geškenbein, Soviet Phys.-JETP 6, 1187 (1958).

GE 59 a B. V. Geškenbein, S. A. Nemirovskaya, and A. P. Rudik, Nuclear Phys. 13, 60 (1959).

GE $59 \mathrm{~b}$ B. V. Geškenbein, S. A. Nemirovskaya, and A. P. Rudik, Zhur. Eksptl. i. Teort. Fiz. 36, 517 (1959).

GO 28 W. Gordon, Z. Physik 48, 180 (1928).

GR 58

GR 51

R. L. Graham, J. S. Geiger, and T. A. Eastwood, Can. J. Phys. 36, 1084 (1958).

E. Greuling and M. L. Meeks, Phys. Rev. 82, 531 (1951).

HA 53

HA 60

D. R. Hamilton, A. Lemonick, and F. M. Pipkin, Phys. Rev. 92, 1191 (1953).

G. Hartwig and H. Schopper, Phys. Rev. Letters 4, $293(1960)$.

HE 54

IB 58

JA 58

R. F. Herbst, Phys. Rev. 96, 372 (1954).

I. Iben, Jr., Phys. Rev. 112, 1240 (1958).

J. D. Jackson, S. B. Treiman, and H. W. Wyld, Jr., Z. Physik 150, 640 (1958).

KI 54

R. W. King and D. C. Peaslee, Phys. Rev. 94, 1284 (1954).

KON 41

KON 54

KON 59

E. T. Konopinski and G. F. Uhlenbeck, Phys. Rev. 60, 308 (1941).

E. J. Konopinski, Phys. Rev. 94, 492 (1954).

E. J. Konopinski, Ann. Rev. Nuclear Sci. 9, 99 (1959).

KOT $58 \mathrm{a}$

T. Kotani and M. Ross, Phys. Rev. Letters 1, 140 (1958).

KOT $58 \mathrm{~b}$ T. Kotani and M. Ross, Progr. Theoret. Phys. (Kyoto) 20, 643 (1958).

KO'T 59a T. Kotani and M. Ross, Phys. Rev. 113, 622 (1959).

KO'T 59b 'T. Kotani, Phys. Rev. 114, 795 (1959).

KU 59a

KU 59b

B. Kuchowitz, Bull. acad. polon. sci. Classe (III) 7, 289 (1959).

B. Kuchowitz, Bull. acad. polon. sci Classe (III) 7, 85 (1959).

LA 54

J. Laberrigue-Frolow and R. Nataf, J. phys. radium 15,438 (1954).

LA 56 M. J. Laubitz, Proc. Phys. Soc. (London) A69, 789 (1956).

LEE $58 \mathrm{a}$

LEE $58 \mathrm{~b}$

C. E. Lee-Whiting, Can. J. Phys. 36, 252 (1958).

G. E. Lee-Whiting, Can. J. Phys. 36, 1199 (1958).

LEW 57a

LEW $57 \mathrm{~b}$

R. P. Lewis, Phys. Rev. 108, 904 (1957).

R. R. Lewis, Jr. and G. W. Ford, Phys. Rev. 107, 756 (1957).

LO $49 \mathrm{a}$

C. Longmire and H. Brown, Phys. Rev. 75, 264 (1949).

LO $49 \mathrm{~b}$ C. Longmire and H. Brown, Phys. Rev. 75, 1102 (1949).

MAC 52 P. Macklin, L. Lidofsky, and C. S. Wu, Phys. Rev. 87, 391 (1952). 
MAH 52

MAH 59

MAL 51

MAL 52

ME 60

ME 51

MO 53a

MO 53b

MO 53c

MO 58a

MO 58b

MO 60

MOS 51

NA 50

NA 54

NA 56

NE 59

PE 60

PE 53

PL 54

PO 59

PU 51

RO 51

RO 53a

RO 53b

RO 54a

RO $54 \mathrm{~b}$

RO 55

SI 58

SI 60

SK 50
H. M. Mahmoud and E. J. Konopinski, Phys. Rev. SL 56 88,1266 (1952)

H. Mahmoud, Ann. Phys. 7, 429 (1959).

I. Malcolm and C. Strachan, Proc. Cambridge Phil. Soc. 47, 610 (1951).

I. Malcolm, Phil. Mag. 43, 1011 (1952)

H. A. Mehlop, E. D. Lambe, and T. Pond, Bull. Am. Phys. Soc. 5, 9 (1960).

E. Merzbacher, Phys. Rev. 81, 942 (1951).

M. Morita, Progr. Theoret. Phys. (Kyoto) 10, 363 (1953).

M. Morita, Phys. Rev. 90, 1005 (1953).

M. Morita, Progr. Theoret. Phys. (Kyoto) 9, 345 (1953).

M. Morita and R. S. Morita, Phys. Rev. 110, 461 (1958).

M. Morita and R. S. Morita, Phys. Rev. 109, 2048 (1958).

M. Morita, Nuclear Phys. 14, 106 (1960).

S. A. Moszkowski, Phys. Rev. 82, 35 (1951).

R. Nataf, Compt. rend. 230, 532 (1950).

R. Nataf, Compt. rend. 238, 1117 (1954).

R. Nataf, J. phys. radium 17, 480 (1956).

N. Newby, Jr. and E. J. Konopinski, Phys. Rev. 115, 434 (1959).

J. M. Pearson, Can. J. Phys. 38, 148 (1960).

D. C. Peaslee, Phys. Rev. 91, 1447 (1953).

E. A. Plassmann and L. M. Langer, Phys. Rev. 96, 1593 (1954).

F. T. Porter and P. P. Day, Phys. Rev. 114, 1286 (1959).

D. L. Pursey, Phil. Mag. 42, 1193 (1951).

M. E. Rose and D. K. Holmes, Phys. Rev. 83, 190 (1951).

M. E. Rose and C. L. Perry, Phys. Rev. 90, 479 (1953).

M. E. Rose, C. L. Perry, and A. Dismuke, Oak Ridge Natl. Lab. Rept. ORNL-1459 (1953).

M. Rose and R. K. Osborn, Phys. Rev. 93, 1315 (1954).

M. Rose and R. K. Osborn, Phys. Rev. 93, 1326 (1954).

M. E. Rose, Multiple Fields (John Wiley \& Sons, New York, 1955).

P. C. Simms and R. M. Steffen, Phys. Rev. Letters 1, 289 (1958).

P. C. Simms and R. M. Steffen, Phys. Rev. 118, YU 56 $768(1960)$.

T. H. R. Skyrme, Progr. in Nuclear Phys. 115, ZY 56 (1950).

SM 51

TA 60

TA 51
L. A. Sliv and B. A. Volchok, Tables of Coulomb Phases and Amplitudes taking into Account the Finite Nuclear Size [Acad. Si. Press (1956); translated in AEC-tr-2875 (1957)].

A. M. Smith, Phys. Rev. 82, 955 (1951).

SP 52a J. A. Spiers and R. J. Blin-Stoyle, Proc. Phys. Soc. (London) A65, 801 (1952).

SP 52b J. A. Spiers and R. J. Blin-Stoyle, Proc. Phys. Soc. (London) A65, 809 (1952).

ST $60 \mathrm{a}$

ST $60 \mathrm{~b}$

R. M. Steffen, Phys. Rev. Letters 4, 290 (1960).

R. M. Steffen, Phys. Rev. 118, 763 (1960).

R. Stump, Phys. Rev. 86, 249 (1952).

D. Tadić, Nuclear Phys. 18, 138 (1960).

TA 54a

TA $54 \mathrm{~b}$

. Takebs

H. Takebe, Progr. Theoret. Phys. (Kyoto) 12, 747 (1954).

M. Taketani, S. Nakamura, K. Ono, and M. Umezawa, Progr. Theoret. Phys. 6, 286 (1951).

TEN 58

Invited Papers from the Conference on Weak Interactions held at Gatlinburg, Tennessee, October 27-29, 1958, Revs. Modern Phys. 31, 782 ff. (1959).

Vo 57 M. E. Voikhanskii, Zhur. Eksptl. i. Teoret. Fiz. 33, 1004 (1957).

WA 59 A. Wapstra, Nuclear Phys. 9, 519 (1959).

WE 58

H. Wegener, H. Bienlein, and H. V. Issendorf, Phys. Rev. Letters 1, 460 (1958).

WU 53 C. S. Wu, F. Boehm, and E. Nagel, Phys. Rev. 91, 319 (1953)

YA 52a

M. Yamada and M. Morita, Progr. Theoret. Phys. (Kyoto) 8, 431 (1952).

YA 52b. M. Yamada and M. Morita, Progr. Theoret. Phys. (Kyoto) 8, 449 (1952).

YA 53a M. Yamada, Progr. Theoret. Phys. (Kyoto) 9, 268 (1953).

YA 53b M. Yamada, Progr. Theoret. Phys. (Kyoto) 10, 245 (1953).

YA 53c M. Yamada, Progr. Theoret. Phys. (Kyoto) 10, $252(1953)$

M. Yamada, Progr. Theoret. Phys. (Kyoto) 10, 241 (1953)

YO 59 R. C. Young, Phys. Rev. 115, 577 (1959).

J. Yukawa, Progr. Theoret. Phys. (Kyoto) 15, $561(1956)$.

L. N. Zyrianowa, Izvest. Nauk SSSR, Ser. Fiz. $20,1399(1956)$. 